\title{
Pan-cancer analysis of whole genomes
}

https://doi.org/10.1038/s41586-020-1969-6

Received: 29 July 2018

Accepted: 11 December 2019

Published online: 5 February 2020

Open access

\section{The ICGC/TCGA Pan-Cancer Analysis of Whole Genomes Consortium}

Cancer is driven by genetic change, and the advent of massively parallel sequencing has enabled systematic documentation of this variation at the whole-genome scale ${ }^{1-3}$. Here we report the integrative analysis of 2,658 whole-cancer genomes and their matching normal tissues across 38 tumour types from the Pan-Cancer Analysis of Whole Genomes (PCAWG) Consortium of the International Cancer Genome Consortium (ICGC) and The Cancer Genome Atlas (TCGA). We describe the generation of the PCAWG resource, facilitated by international data sharing using compute clouds. On average, cancer genomes contained 4-5 driver mutations when combining coding and non-coding genomic elements; however, in around $5 \%$ of cases no drivers were identified, suggesting that cancer driver discovery is not yet complete. Chromothripsis, in which many clustered structural variants arise in a single catastrophic event, is frequently an early event in tumour evolution; in acral melanoma, for example, these events precede most somatic point mutations and affect several cancer-associated genes simultaneously. Cancers with abnormal telomere maintenance often originate from tissues with low replicative activity and show several mechanisms of preventing telomere attrition to critical levels. Common and rare germline variants affect patterns of somatic mutation, including point mutations, structural variants and somatic retrotransposition. A collection of papers from the PCAWG Consortium describes non-coding mutations that drive cancer beyond those in the TERT promoter ${ }^{4}$; identifies new signatures of mutational processes that cause base substitutions, small insertions and deletions and structural variation ${ }^{5,6}$; analyses timings and patterns of tumour evolution ${ }^{7}$; describes the diverse transcriptional consequences of somatic mutation on splicing, expression levels, fusion genes and promoter activity ${ }^{8,9}$; and evaluates a range of more-specialized features of cancer genomes ${ }^{8,10-18}$.
Cancer is the second most-frequent cause of death worldwide, killing more than 8 million people every year; the incidence of cancer is expected to increase by more than $50 \%$ over the coming decades ${ }^{19,20}$. 'Cancer' is a catch-all term used to denote a set of diseases characterized by autonomous expansion and spread of a somatic clone. To achieve this behaviour, the cancer clone must co-opt multiple cellular pathways that enable it to disregard the normal constraints on cell growth, modify the local microenvironment to favour its own proliferation, invade through tissue barriers, spread to other organs and evade immune surveillance $^{21}$. No single cellular program directs these behaviours. Rather, there is a large pool of potential pathogenic abnormalities from which individual cancers draw their own combinations: the commonalities of macroscopic features across tumours belie a vastly heterogeneous landscape of cellular abnormalities.

This heterogeneity arises from the stochastic nature of Darwinian evolution. There are three preconditions for Darwinian evolution: characteristics must vary within a population; this variation must be heritable from parent to offspring; and there must be competition for survival within the population. In the context of somatic cells, heritable variation arises from mutations acquired stochastically throughout life, notwithstanding additional contributions from germline and epigenetic variation. A subset of these mutations alter the cellular phenotype, and a small subset of those variants confer an advantage on clones during the competition to escape the tight physiological controls wired into somatic cells. Mutations that provide a selective advantage to the clone are termed driver mutations, as opposed to selectively neutral passenger mutations.

Initial studies using massively parallel sequencing demonstrated the feasibility of identifying every somatic point mutation, copy-number change and structural variant (SV) in a given cancer ${ }^{1-3}$. In 2008, recognizing the opportunity that this advance in technology provided, the global cancer genomics community established the ICGC with the goal of systematically documenting the somatic mutations that drive common tumour types ${ }^{22}$.

\section{The pan-cancer analysis of whole genomes}

The expansion of whole-genome sequencing studies from individual ICGC and TCGA working groups presented the opportunity to undertake a meta-analysis of genomic features across tumour types. To achieve this, the PCAWG Consortium was established. A Technical Working Group implemented the informatics analyses by aggregating the raw sequencing data from different working groups that studied individual tumour types, aligning the sequences to the human genome and delivering a set of high-quality somatic mutation calls for downstream analysis (Extended Data Fig. 1). Given the recent meta-analysis 


\section{Online resources for data access, visualization and analysis}

The PCAWG landing page (http://docs.icgc.org/pcawg) provides links to several data resources for interactive online browsing, analysis and download of PCAWG data and results (Supplementary Table 4).

\section{Direct download of PCAWG data}

Aligned PCAWG read data in BAM format are also available at the European Genome Phenome Archive (EGA; https://www. ebi.ac.uk/ega/search/site/pcawg under accession number EGAS00001001692). In addition, all open-tier PCAWG genomics data, as well as reference datasets used for analysis, can be downloaded from the ICGC Data Portal at http://docs.icgc.org/ pcawg/data/. Controlled-tier genomic data, including SNVs and indels that originated from TCGA projects (in VCF format) and aligned reads (in BAM format) can be downloaded using the Score (https://www.overture.bio/) software package, which has accelerated and secure file transfer, as well as BAM slicing facilities to selectively download defined regions of genomic alignments.

\section{PCAWG computational pipelines}

The core alignment, somatic variant-calling, quality-control and variant consensus-generation pipelines used by PCAWG have each been packaged into portable cross-platform images using the Dockstore system ${ }^{84}$ and released under an Open Source licence that enables unrestricted use and redistribution. All PCAWG Dockstore images are available to the public at https://dockstore.org/ organizations/PCAWG/collections/PCAWG.

\section{ICGC Data Portal}

The ICGC Data Portal ${ }^{85}$ (https://dcc.icgc.org) serves as the main entry point for accessing PCAWG datasets with a single uniform web interface and a high-performance data-download client. This uniform interface provides users with easy access to the myriad of PCAWG sequencing data and variant calls that reside in many repositories and compute clouds worldwide. Streaming technology ${ }^{86}$ provides users with high-level visualizations in real time of BAM and VCF files stored remotely on the Cancer Genome Collaboratory.

\section{Ucsc Xena}

UCSC Xena ${ }^{87}$ (https://pcawg.xenahubs.net) visualizes all PCAWG primary results, including copy-number, gene-expression, gene-fusion and promoter-usage alterations, simple somatic mutations, large somatic structural variations, mutational signatures and phenotypic data. These open-access data are available through a public Xena hub, and consensus simple somatic mutations can be loaded to the local computer of a user via a private Xena hub. Kaplan-Meier plots, histograms, box plots, scatter plots and transcript-specific views offer additional visualization options and statistical analyses.

\section{The Expression Atlas}

The Expression Atlas (https://www.ebi.ac.uk/gxa/home) contains RNA-sequencing and expression microarray data for querying gene expression across tissues, cell types, developmental stages and/or experimental conditions ${ }^{88}$. Two different views of the data are provided: summarized expression levels for each tumour type and gene expression at the level of individual samples, including reference-gene expression datasets for matching normal tissues.

\section{PCAWG Scout}

PCAWG Scout (http://pcawgscout.bsc.es/) provides a framework for -omics workflow and website templating to generate on-demand, in-depth analyses of the PCAWG data that are openly available to the whole research community. Views of protected data are available that still safeguard sensitive data. Through the PCAWG Scout web interface, users can access an array of reports and visualizations that leverage on-demand bioinformatic computing infrastructure to produce results in real time, allowing users to discover trends as well as form and test hypotheses.

\section{Chromothripsis Explorer}

Chromothripsis Explorer (http://compbio.med.harvard.edu/ chromothripsis/) is a portal that allows structural variation in the PCAWG dataset to be explored on an individual patient basis through the use of circos plots. Patterns of chromothripsis can also be explored in aggregated formats. of exome data from the TCGA Pan-Cancer Atlas ${ }^{23-25}$, scientific working groups concentrated their efforts on analyses best-informed by wholegenome sequencing data.

We collected genome data from 2,834 donors (Extended Data Table 1), of which 176 were excluded after quality assurance. A further 75 had minor issues that could affect some of the analyses (grey-listed donors) and 2,583 had data of optimal quality (white-listed donors) (Supplementary Table1). Across the 2,658 white- and grey-listed donors, whole-genome sequencing data were available from 2,605 primary tumours and 173 metastases or local recurrences. Mean read coverage was $39 \times$ for normal samples, whereas tumours had a bimodal coverage distribution with modes at $38 \times$ and $60 \times$ (Supplementary Fig. 1 ). RNA-sequencing data were available for 1,222 donors. The final cohort comprised 1,469 men (55\%) and 1,189 women (45\%), with a mean age of 56 years (range, $1-90$ years) across 38 tumour types (Extended Data Table 1 and Supplementary Table 1).

To identify somatic mutations, we analysed all 6,835 samples using a uniform set of algorithms for alignment, variant calling and quality control (Extended Data Fig. 1,Supplementary Fig. 2 and Supplementary Methods 2). We used three established pipelines to call somatic singlenucleotide variations (SNVs), small insertions and deletions (indels), copy-number alterations (CNAs) and SVs. Somatic retrotransposition events, mitochondrial DNA mutations and telomere lengths were also called by bespoke algorithms. RNA-sequencing data were uniformly processed to call transcriptomic alterations. Germline variants identified by the three separate pipelines included single-nucleotide polymorphisms, indels, SVs and mobile-element insertions (Supplementary Table 2).

The requirement to uniformly realign and call variants on approximately 5,800 whole genomes presented considerable computational challenges, and raised ethical issues owing to the use of data from different jurisdictions (Extended Data Table 2). We used cloud computing $^{26,27}$ to distribute alignment and variant calling across 13 data centres on 3 continents (Supplementary Table 3 ). Core pipelines were packaged into Docker containers ${ }^{28}$ as reproducible, stand-alone packages, which we have made available for download. Data repositories for raw and derived datasets, together with portals for data visualization and exploration, have also been created (Box 1 and Supplementary Table 4).

\section{Benchmarking of genetic variant calls}

To benchmark mutation calling, we ran the 3 core pipelines, together with 10 additional pipelines, on 63 representative tumour-normal genome pairs (Supplementary Note 1). For 50 of these cases, we performed validation by hybridization of tumour and matched normal DNA to a custom bait set with deep sequencing ${ }^{29}$. The 3 core somatic variantcalling pipelines had individual estimates of sensitivity of $80-90 \%$ to detect a true somatic SNV called by any of the 13 pipelines; more 


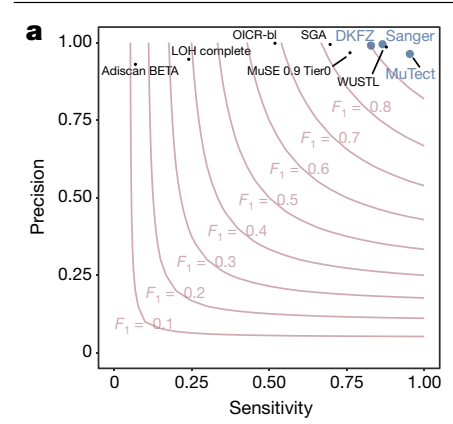

c - DKFZ - Sanger - two_plus
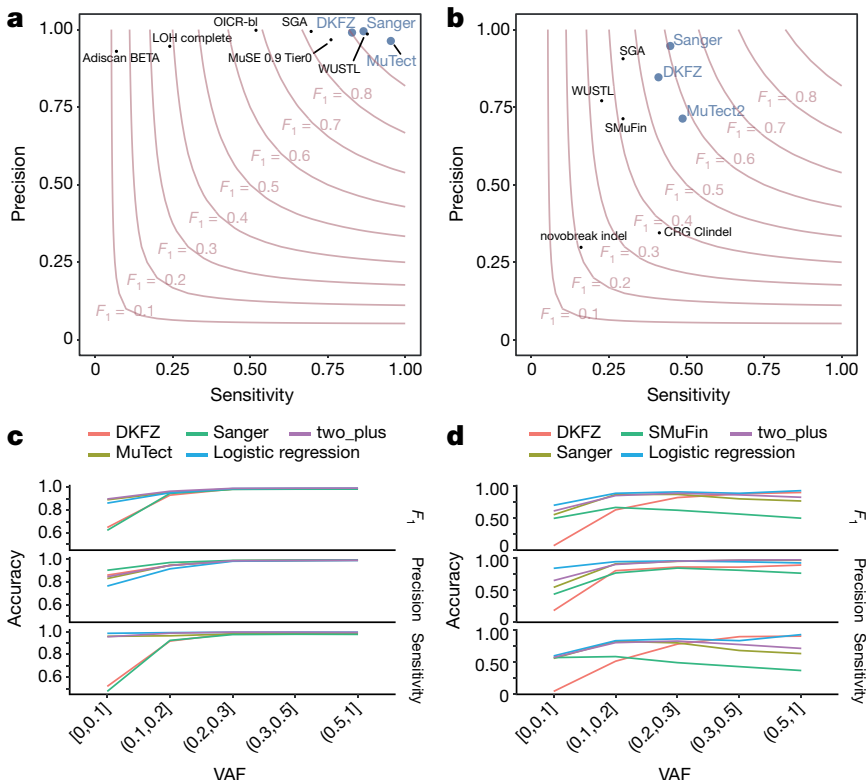

d - DKFZ - SMuFin - two_plus - Sanger — Logistic regression

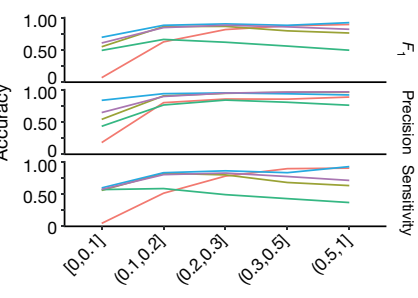

VAF

Fig. 1 | Validation of variant-calling pipelines in PCAWG. a, Scatter plot of estimated sensitivity and precision for somatic SNVs across individual algorithms assessed in the validation exercise across $n=63$ PCAWG samples. Core algorithms included in the final PCAWG call set are shown in blue. b, Sensitivity and precision estimates across individual algorithms for somatic indels. c, Accuracy (precision, sensitivity and $F_{1}$ score, defined as $2 \times$ sensitivity $\times$ precision/(sensitivity + precision)) of somatic SNV calls across variant allele fractions (VAFs) for the core algorithms. The accuracy of two methods of combining variant calls (two-plus, which was used in the final dataset, and logistic regression) is also shown. d, Accuracy of indel calls across variant allele fractions.

than $95 \%$ of SNV calls made by each of the core pipelines were genuine somatic variants (Fig. 1a). For indels-a more-challenging class of variants to identify with short-read sequencing-the 3 core algorithms had individual sensitivity estimates in the range of $40-50 \%$, with precision of 70-95\% (Fig. 1b). For individual SV algorithms, we estimated precision to be in the range $80-95 \%$ for samples in the 63 -sample pilot dataset.

Next, we defined a strategy to merge results from the three pipelines into one final call-set to be used for downstream scientific analyses (Methods and Supplementary Note 2). Sensitivity and precision of consensus somatic variant calls were $95 \%$ (90\% confidence interval, 88-98\%) and 95\% (90\% confidence interval, 71-99\%), respectively, for SNVs (Extended Data Fig. 2). For somatic indels, sensitivity and precision were 60\% (34-72\%) and 91\% (73-96\%), respectively (Extended Data Fig. 2). Regarding somatic SVs, we estimate the sensitivity of merged calls to be $90 \%$ for true calls generated by any one pipeline; precision was estimated as $\mathbf{9 7 . 5 \%}$. The improvement in calling accuracy from combining different pipelines was most noticeable in variants with low variant allele fractions, which probably originate from tumour subclones (Fig. 1c, d). Germline variant calls, phased using a haplotypereference panel, displayed a precision of more than $99 \%$ and a sensitivity of 92-98\% (Supplementary Note 2).

\section{Analysis of PCAWG data}

The uniformly generated, high-quality set of variant calls across more than 2,500 donors provided the springboard for a series of scientific working groups to explore the biology of cancer. A comprehensive suite of companion papers that describe the analyses and discoveries across these thematic areas is copublished with this paper ${ }^{4-18}$ (Extended Data Table 3).

\section{Pan-cancer burden of somatic mutations}

Across the 2,583 white-listed PCAWG donors, we called 43,778,859 somatic SNVs, 410,123 somatic multinucleotide variants, 2,418,247 somatic indels, 288,416 somatic SVs, 19,166 somatic retrotransposition events and 8,185 de novo mitochondrial DNA mutations (Supplementary Table 1). There was considerable heterogeneity in the burden of somatic mutations across patients and tumour types, with a broad correlation in mutation burden among different classes of somatic variation (Extended Data Fig. 3). Analysed at a per-patient level, this correlation held, even when considering tumours with similar purity and ploidy (Supplementary Fig. 3). Why such correlation should apply on a pan-cancer basis is unclear. It is likely that age has some role, as we observe a correlation between most classes of somatic mutation and age at diagnosis (around $190 \mathrm{SNV}$ s per year, $P=0.02$; about 22 indels per year, $P=5 \times 10^{-5} ; 1.5 \mathrm{SV}$ s per year, $P<2 \times 10^{-16}$; linear regression with likelihood ratio tests; Supplementary Fig. 4 ). Other factors are also likely to contribute to the correlations among classes of somatic mutation, as there is evidence that some DNA-repair defects can cause multiple types of somatic mutation ${ }^{30}$, and a single carcinogen can cause a range of DNA lesions ${ }^{31}$.

\section{Panorama of driver mutations in cancer}

We extracted the subset of somatic mutations in PCAWG tumours that have high confidence to be driver events on the basis of current knowledge. One challenge to pinpointing the specific driver mutations in an individual tumour is that not all point mutations in recurrently mutated cancer-associated genes are drivers ${ }^{32}$. For genomic elements significantly mutated in PCAWG data, we developed a 'rankand-cut' approach to identify the probable drivers (Supplementary Methods 8.1). This approach works by ranking the observed mutations in a given genomic element based on recurrence, estimated functional consequence and expected pattern of drivers in that element. We then estimate the excess burden of somatic mutations in that genomic element above that expected for the background mutation rate, and cut the ranked mutations at this level. Mutations in each element with the highest driver ranking were then assigned as probable drivers; those below the threshold will probably have arisen through chance and were assigned as probable passengers. Improvements to features that are used to rank the mutations and the methods used to measure them will contribute to further development of the rank-and-cut approach.

We also needed to account for the fact that some bona fide cancer genomic elements were not rediscovered in PCAWG data because of low statistical power. We therefore added previously known cancer-associated genes to the discovery set, creating a 'compendium of mutational driver elements' (Supplementary Methods 8.2). Then, using stringent rules to nominate driver point mutations that affect these genomic elements on the basis of prior knowledge $\mathrm{e}^{33}$, we separated probable driver from passenger point mutations. To cover all classes of variant, we also created a compendium of known driver SVs, using analogous rules to identify which somatic CNAs and SVs are most likely to act as drivers in each tumour. For probable pathogenic germline variants, we identified all truncating germline point mutations and SVs that affect high-penetrance germline cancer-associated genes.

This analysis defined a set of mutations that we could confidently assert, based on current knowledge, drove tumorigenesis in the more than 2,500 tumours of PCAWG. We found that $91 \%$ of tumours had at least one identified driver mutation, with an average of 4.6 drivers per tumour identified, showing extensive variation across cancer types (Fig. 2a). For coding point mutations, the average was 2.6 drivers per tumour, similar to numbers estimated in known cancer-associated genes in tumours in the TCGA using analogous approaches ${ }^{32}$.

To address the frequency of non-coding driver point mutations, we combined promoters and enhancers that are known targets of 

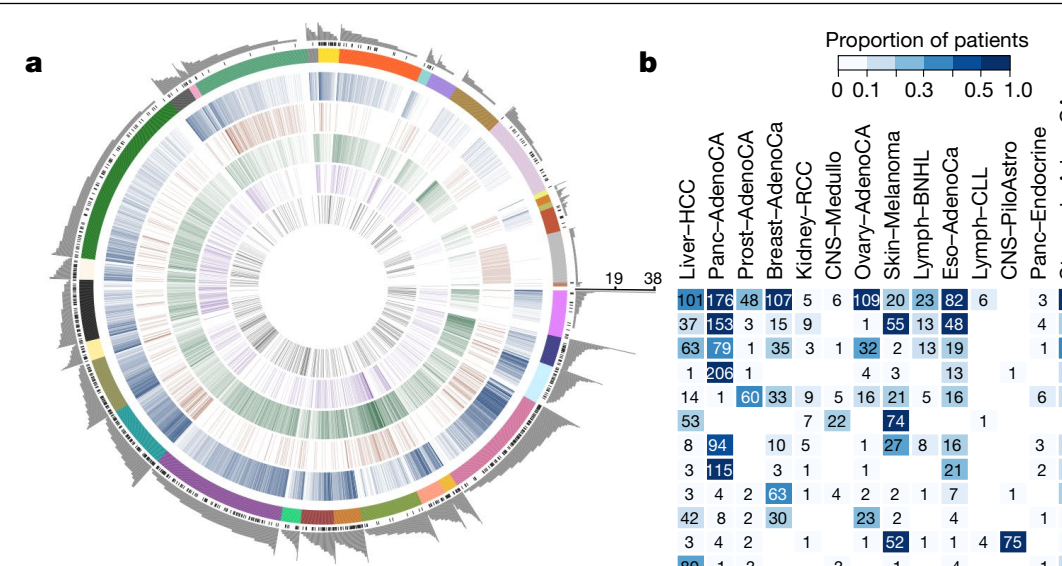

\begin{tabular}{ll}
\multicolumn{2}{c}{ Mutations } \\
\hline Coding Promoter & $5^{\prime}$ UTR \\
Intron splicing & 3' UTR
\end{tabular}
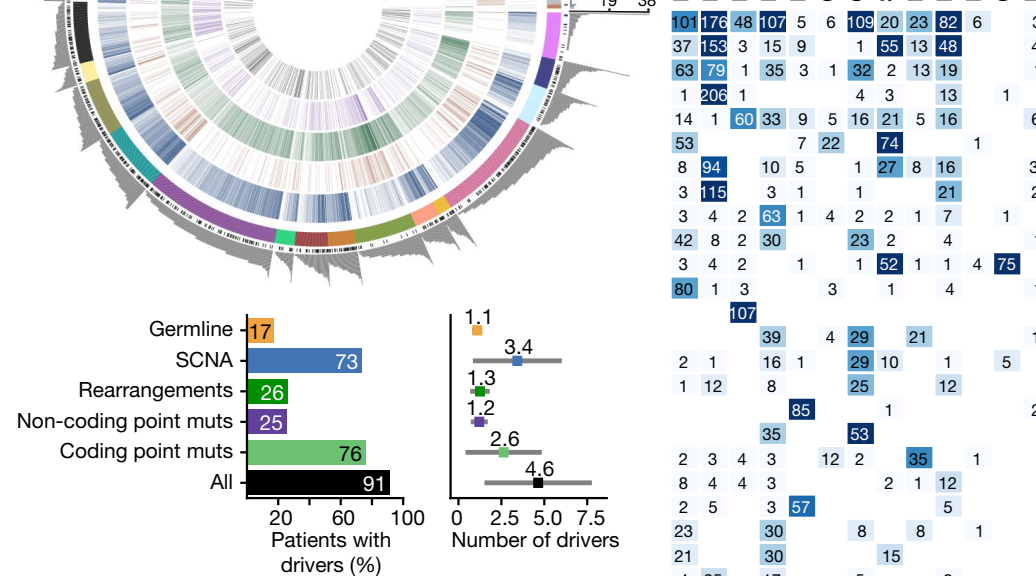

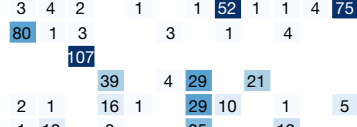
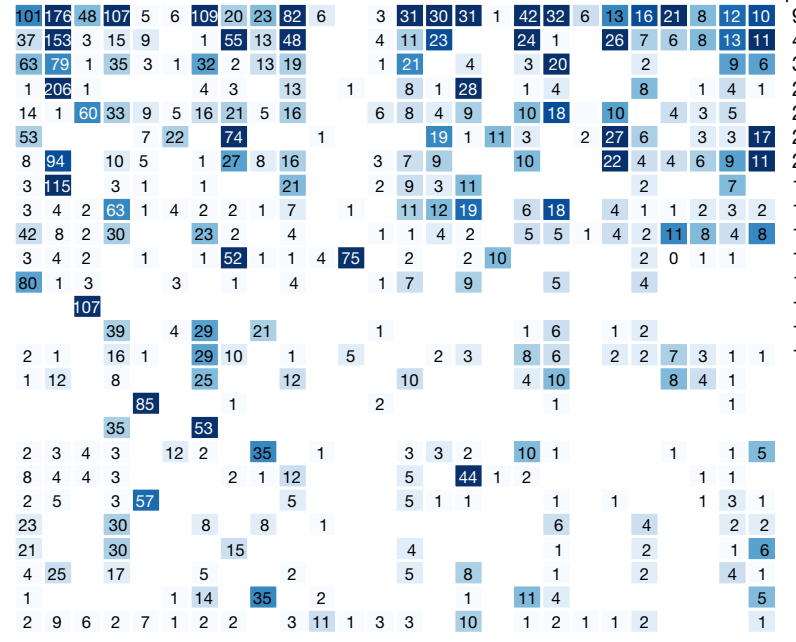
$\begin{array}{lllllllll}21 & 4 & 3 & 20 & 2 & 9 & 6 & 316 & \text { ARID1A }\end{array}$ $\begin{array}{lllllllllll}8 & 1 & 28 & 1 & 4 & 8 & 1 & 4 & 1 & 287 & \text { KRAS }\end{array}$ $\begin{array}{llllllllll}4 & 9 & 10 & 18 & 10 & 4 & 3 & 5 & 269 & \text { PTEN }\end{array}$ $\begin{array}{lllllllllllll}19 & 1 & 11 & 3 & 2 & 27 & 6 & 3 & 3 & 17 & 263 & \text { TERT }\end{array}$

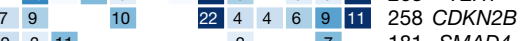
\begin{tabular}{l|l|l|l|l|l|l}
9 & 3 & 11 & 2 & 7 & 181 & $S M A D 4$
\end{tabular} \begin{tabular}{ll|llllllllllll}
11 & 12 & 19 & 6 & 18 & 4 & 1 & 1 & 2 & 3 & 2 & 177 & PIK3CA
\end{tabular} \begin{tabular}{llllll|lllllllllll}
1 & 1 & 4 & 2 & 5 & 5 & 1 & 4 & 2 & 11 & 8 & 4 & 8 \\
\hline
\end{tabular} $\begin{array}{llllll}2 & 0 & 1 & 1 & 162 & B R A F\end{array}$ 4118 CTNNB1 118 CTNNB
107 ERG $\begin{array}{ll}107 & E R G \\ 106 & M Y C \\ 103 & N F 1\end{array}$ 2 \begin{tabular}{ll|l|l|l|}
2 & 7 & 3 & 1
\end{tabular} $\begin{array}{cc}103 & N F 1 \\ 95 & C C N E 1 \\ 90 & \text { VHL }\end{array}$ $90 \mathrm{VHL}$ 90 19p13.3a $\begin{array}{llllll}3 & 3 & 2 & 10 & 1\end{array}$ $\begin{array}{lcccc}5 & & 44 & 1 & 2\end{array}$ $\begin{array}{lllll}5 & 1 & 1 & 1 & 1\end{array}$ 4
5 \begin{tabular}{|lr|r} 
& 1 \\
8 & 1 \\
1 & 11 & 4 \\
10 & 1 &
\end{tabular} 88 APC 85 PBRM1 84 MCL1 $\begin{array}{llll}2 & 2 & 84 & \text { MCL1 } \\ 1 & 6 & 83 & \text { CCND1 }\end{array}$

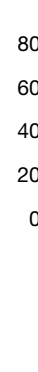

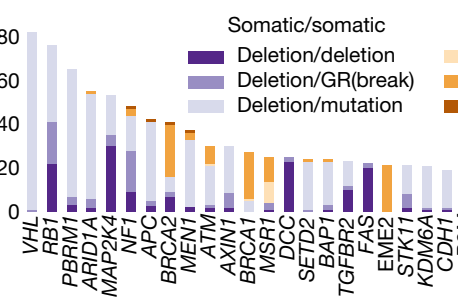

Somatic/somatic
Deletion/deletion
Deletion/GR(break)
Deletion/mutation

Germline/somatic

Deletion/deletion

Mutation/deletion

Mutation/mutation
Fig. 2 | Panorama of driver mutations in PCAWG. a, Top, putative driver mutations in PCAWG, represented as a circos plot. Each sector represents a tumour in the cohort. From the periphery to the centre of the plot the concentric rings represent: (1) the total number of driver alterations; (2) the presence of whole-genome (WG) duplication; (3) the tumour type; (4) the number of driver CNAs; (5) the number of driver genomic rearrangements; (6) driver coding point mutations; (7) driver non-coding point mutations; and (8) pathogenic germline variants. Bottom, snapshots of the panorama of driver mutations. The horizontal bar plot (left) represents the proportion of patients with different types of drivers. The dot plot (right) represents the mean number of each type of driver mutation across tumours with at least one event (the square dot) and the standard deviation (grey whiskers), based on $n=2,583$

non-coding drivers ${ }^{34-37}$ with those newly discovered in PCAWG data; this is reported in a companion paper ${ }^{4}$. Using this approach, only $13 \%(785$ out of 5,913$)$ of driver point mutations were non-coding in PCAWG. Nonetheless, 25\% of PCAWG tumours bear at least one putative non-coding driver point mutation, and one third (237 out of 785) affected the TERT promoter (9\% of PCAWG tumours). Overall, non-coding driver point mutations are less frequent than coding driver mutations. With the exception of the TERT promoter, individual enhancers and promoters are only infrequent targets of driver mutations ${ }^{4}$.

Across tumour types, SVs and point mutations have different relative contributions to tumorigenesis. Driver SVs are more prevalent in breast adenocarcinomas $(6.4 \pm 3.7 \mathrm{SVs}$ (mean \pm s.d.) compared with $2.2 \pm 1.3$ point mutations; $P<1 \times 10^{-16}$, Mann-Whitney $U$-test) and ovary adenocarcinomas $(5.8 \pm 2.6$ SVs compared with $1.9 \pm 1.0$ point mutations; $\left.P<1 \times 10^{-16}\right)$, whereas driver point mutations have patients. b, Genomic elements targeted by different types of mutations in the cohort altered in more than 65 tumours. Both germline and somatic variants are included. Left, the heat map shows the recurrence of alterations across cancer types. The colour indicates the proportion of mutated tumours and the number indicates the absolute count of mutated tumours. Right, the proportion of each type of alteration that affects each genomic element. c, Tumour-suppressor genes with biallelic inactivation in 10 or more patients. The values included under the gene labels represent the proportions of patients who have biallelic mutations in the gene out of all patients with a somatic mutation in that gene. GR, genomic rearrangement; SCNA, somatic copy-number alteration; SGR, somatic genome rearrangement; TSG, tumour suppressor gene; UTR, untranslated region.

a larger contribution in colorectal adenocarcinomas $(2.4 \pm 1.4 \mathrm{SVs}$ compared with $7.4 \pm 7.0$ point mutations; $P=4 \times 10^{-10}$ ) and mature B cell lymphomas $(2.2 \pm 1.3 \mathrm{SVs}$ compared with $6 \pm 3.8$ point mutations; $P<1 \times 10^{-16}$ ), as previously shown ${ }^{38}$. Across tumour types, there are differences in which classes of mutation affect a given genomic element (Fig. 2b).

We confirmed that many driver mutations that affect tumoursuppressor genes are two-hit inactivation events (Fig. 2c). For example, of the 954 tumours in the cohort with driver mutations in TP53, 736 (77\%) had both alleles mutated, $96 \%$ of which (707 out of 736 ) combined a somatic point mutation that affected one allele with somatic deletion of the other allele. Overall, $17 \%$ of patients had rare germline protein-truncating variants (PTVs) in cancer-predisposition genes ${ }^{39}$, DNA-damage response genes ${ }^{40}$ and somatic driver genes. Biallelic inactivation due to somatic alteration on top of a germline PTV was observed in $4.5 \%$ of patients overall, with $81 \%$ of 
a

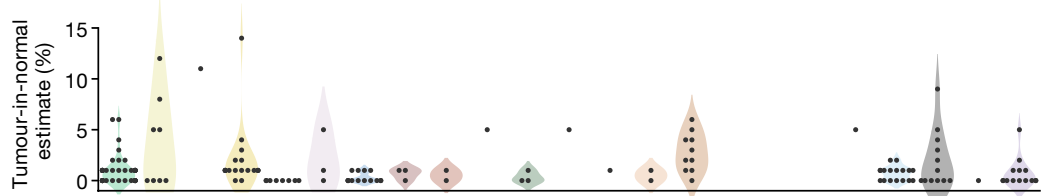

b

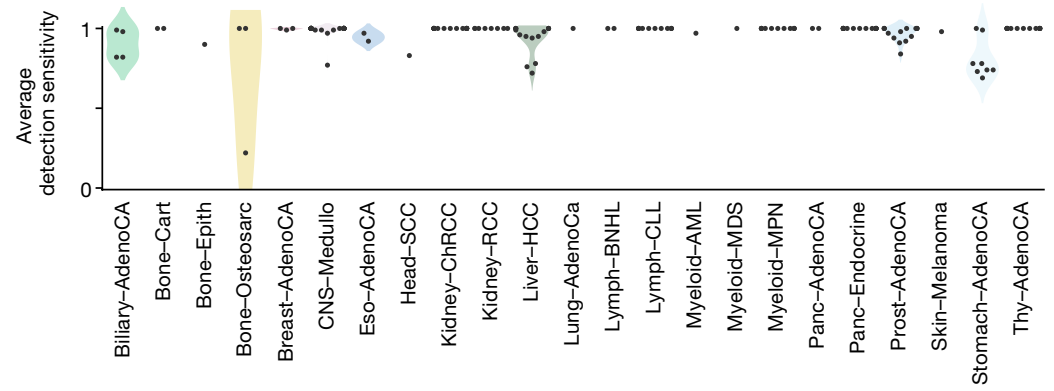

Chromosome 5: 1,259,228

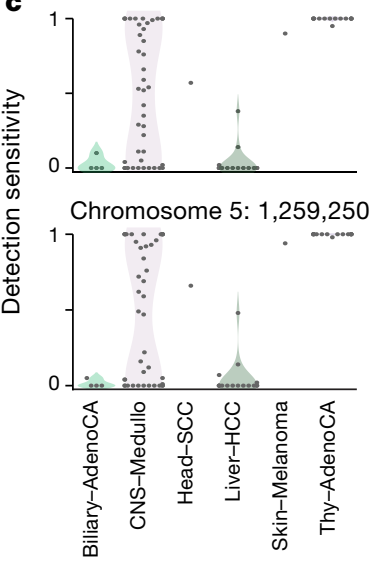

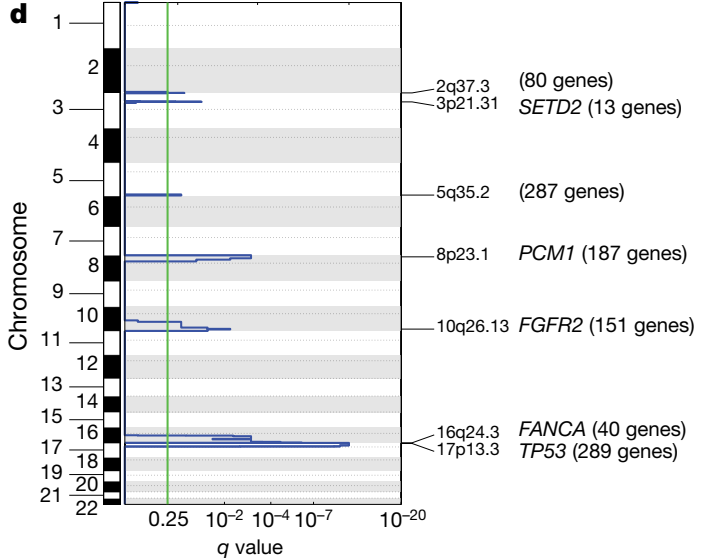

Fig. 3 | Analysis of patients with no detected driver mutations. a, Individual estimates of the percentage of tumour-in-normal contamination across patients with no driver mutations in PCAWG $(n=181)$. No data were available for myelodysplastic syndromes and acute myeloid leukaemia. Points represent estimates for individual patients, and the coloured areas are estimated density distributions (violin plots). Abbreviations of the tumour types are defined in Extended Data Table 1.b, Average detection sensitivity by tumour type for tumours without known drivers $(n=181)$. Each dot represents a given sample and is the average sensitivity of detecting clonal substitutions across the genome, taking into account purity and ploidy. Coloured areas are estimated density distributions, shown for cohorts with at least five cases. c, Detection sensitivity for TERT promoter hotspots in tumour types in which TERT is frequently mutated. Coloured areas are estimated density distributions. d, Significant copy-number losses identified by two-sided hypothesis testing using GISTIC2.0, corrected for multiple-hypothesis testing. Numbers in parentheses indicate the number of genes in significant regions when analysing medulloblastomas without known drivers $(n=42)$. Significant regions with known cancer-associated genes are labelled with the representative cancer-associated gene.e, Aneuploidy in chromophobe renal cell carcinomas and pancreatic neuroendocrine tumours without known drivers. Patients are ordered on the $y$ axis by tumour type and then by presence of whole-genome duplication (bottom) or not (top). these affecting known cancer-predisposition genes (such as BRCA1, $B R C A 2$ and $A T M)$.

\section{PCAWG tumours with no apparent drivers}

Although more than $90 \%$ of PCAWG cases had identified drivers, we found none in 181 tumours (Extended Data Fig. 4a). Reasons for missing drivers have not yet been systematically evaluated in a pan-cancer cohort, and could arise from either technical or biological causes.

Technical explanations could include poor-quality samples, inadequate sequencing or failures in the bioinformatic algorithms used. We assessed the quality of the samples and found that 4 of the 181 cases with no known drivers had more than $5 \%$ tumour DNA contamination in their matched normal sample (Fig. 3a). Using an algorithm designed to correct for this contamination ${ }^{41}$, we identified previously missed mutations in genes relevant to the respective cancer types. Similarly, if the fraction of tumour cells in the cancer sample is low through stromal contamination, the detection of driver mutations can be impaired. Most tumours with no known drivers had an average power to detect mutations close to $100 \%$; however, a few had power in the $70-90 \%$ range (Fig. $3 \mathrm{~b}$ and Extended Data Fig. 4b). Even in adequately sequenced genomes, lack of read depth at specific driver loci can impair mutation detection. For example, only around $50 \%$ of PCAWG tumours had sufficient coverage to call a mutation ( $\geq 90 \%$ power) at the two TERT promoter hotspots, probably because the high GC content of this region causes biased coverage (Fig. $3 \mathrm{C}$ ). In fact, 6 hepatocellular carcinomas and 2 biliary cholangiocarcinomas among the 181 cases with no known drivers actually did contain TERT mutations, which were discovered after deep targeted sequencing ${ }^{42}$.

Finally, technical reasons for missing driver mutations include failures in the bioinformatic algorithms. This affected 35 myeloproliferative neoplasms in PCAWG, in which the $J A K 2^{V 617 F}$ driver mutation should have been called. Our somatic variant-calling algorithms rely on 'panels of normals', typically from blood samples, to remove recurrent sequencing artefacts. As $2-5 \%$ of healthy individuals carry occult haematopoietic clones ${ }^{43}$, recurrent driver mutations in these clones can enter panels of normals.

With regard to biological causes, tumours may be driven by mutations in cancer-associated genes that are not yet described for that tumour type. Using driver discovery algorithms on tumours with no known drivers, no individual genes reached significance for point mutations. However, we identified a recurrent CNA that spanned SETD2 in 


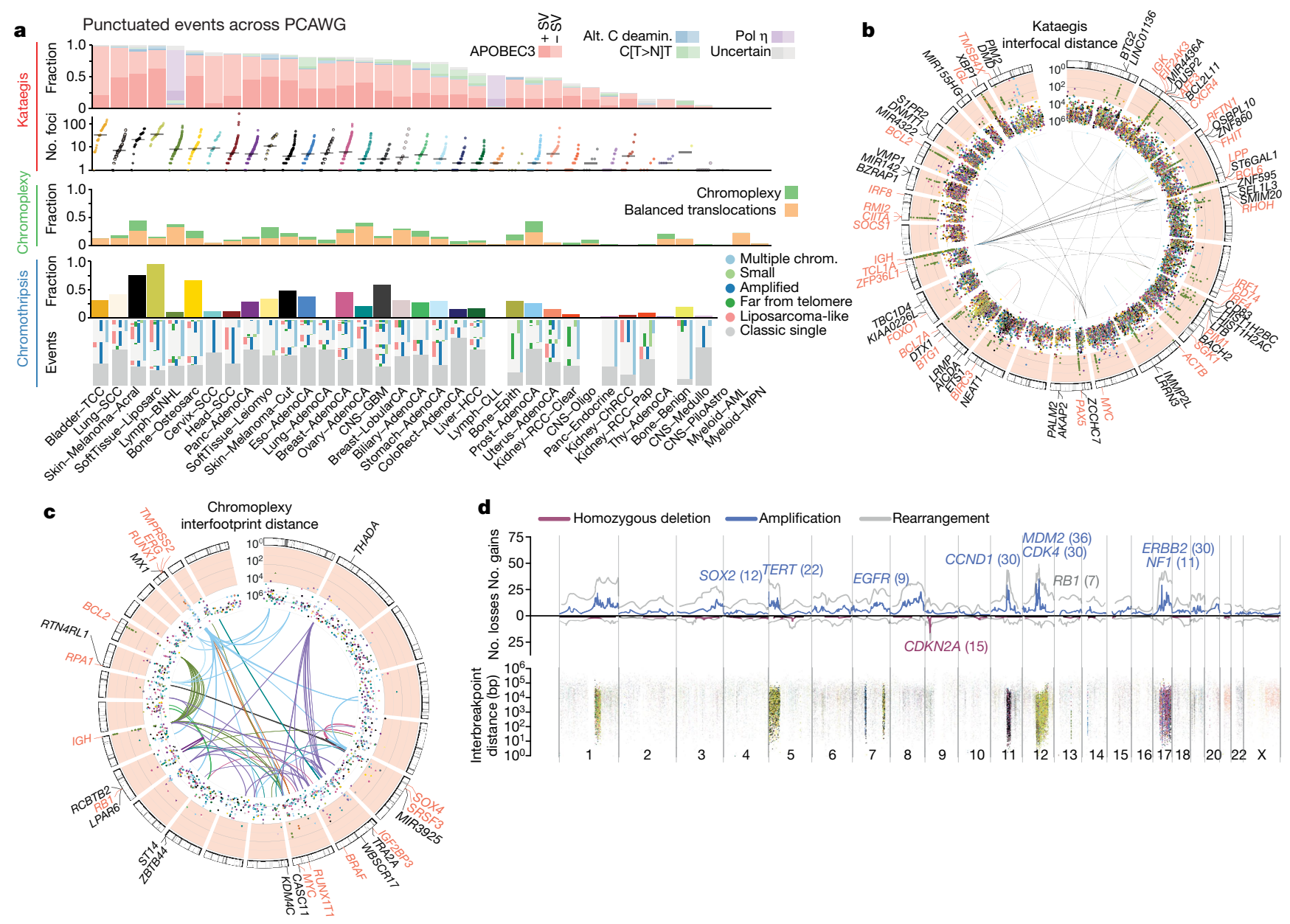

Fig. 4 | Patterns of clustered mutational processes in PCAWG. a, Kataegis. Top, prevalence of different types of kataegis and their association with SVs ( $\leq 1 \mathrm{~kb}$ from the focus). Bottom, the distribution of the number of foci of kataegis per sample. Chromoplexy. Prevalence of chromoplexy across cancer types, subdivided into balanced translocations and more complex events. Chromothripsis. Top, frequency of chromothripsis across cancer types. Bottom, for each cancer type a column is shown, in which each row is a chromothripsis region represented by five coloured rectangles relating to its categorization. b. Circos rainfall plot showing the distances between consecutive kataegis events across PCAWG compared with their genomic position. Lymphoid tumours (khaki, B cell non-Hodgkin's lymphoma; orange, chronic lymphocytic leukaemia) have hypermutation hot spots ( $\geq 3$ foci with distance $\leq 1 \mathrm{~kb}$; pale red zone), many of which are near known cancer-associated genes (red annotations) and have associated SVs ( $\leq 10 \mathrm{~kb}$ from the focus; shown as arcs in the centre). c, Circos rainfall plot as in b that shows the distance versus the position of consecutive chromoplexy and reciprocal translocation footprints across PCAWG. Lymphoid, prostate and thyroid cancers exhibit recurrent events ( $\geq 2$ footprints with distance $\leq 10 \mathrm{~kb}$; pale red zone) that are likely to be driver SVs and are annotated with nearby genes and associated SVs, which are shown as bold and thin arcs for chromoplexy and reciprocal translocations, respectively (colours as in a). d, Effect of chromothripsis along the genome and involvement of PCAWG driver genes. Top, number of chromothripsis-induced gains or losses (grey) and amplifications (blue) or deletions (red). Within the identified chromothripsis regions, selected recurrently rearranged (light grey), amplified (blue) and homozygously deleted (magenta) driver genes are indicated. Bottom, interbreakpoint distance between all subsequent breakpoints within chromothripsis regions across cancer types, coloured by cancer type. Regions with an average interbreakpoint distance $<10 \mathrm{~kb}$ are highlighted. $\mathrm{C}[\mathrm{T}>\mathrm{N}] \mathrm{T}$, kataegis with a pattern of thymine mutations in a Cp TpT context. medulloblastomas that lacked known drivers (Fig. 3d), indicating that restricting hypothesis testing to missing-driver cases can improve power if undiscovered genes are enriched in such tumours. Inactivation of SETD2 in medulloblastoma significantly decreased gene expression $(P=0.002)$ (Extended Data Fig. 4c). Notably, SETD2 mutations occurred exclusively in medulloblastoma group-4 tumours $\left(P<1 \times 10^{-4}\right)$. Group-4 medulloblastomas are known for frequent mutations in other chromatin-modifying genes ${ }^{44}$, and our results suggest that SETD2 loss of function is an additional driver that affects chromatin regulators in this subgroup.

Two tumour types had a surprisingly high fraction of patients without identified driver mutations: chromophobe renal cell carcinoma $(44 \%$; 19 out of 43$)$ and pancreatic neuroendocrine cancers $(22 \%$; 18 out of 81 ) (Extended Data Fig. 4a). A notable feature of the missing-driver cases in both tumour types was a remarkably consistent profile of chromosomal aneuploidy-patterns that have previously been reported ${ }^{45,46}$ (Fig. 3e). The absence of other identified driver mutations in these patients raises the possibility that certain combinations of whole-chromosome gains and losses may be sufficient to initiate a cancer in the absence of more-targeted driver events such as point mutations or fusion genes of focal CNAs.

Even after accounting for technical issues and novel drivers, 5.3\% of PCAWG tumours still had no identifiable driver events. In a research setting, in which we are interested in drawing conclusions about populations of patients, the consequences of technical issues that affect occasional samples will be mitigated by sample size. In a clinical setting, in which we are interested in the driver mutations in a specific patient, these issues become substantially more important. Careful and critical appraisal of the whole pipeline-including sample acquisition, genome sequencing, mapping, variant calling and driver annotation, as done 
here-should be required for laboratories that offer clinical sequencing of cancer genomes.

\section{Patterns of clustered mutations and SVs}

Some somatic mutational processes generate multiple mutations in a single catastrophic event, typically clustered in genomic space, leading to substantial reconfiguration of the genome. Three such processes have previously been described: (1) chromoplexy, in which repair of co-occurring double-stranded DNA breaks-typically on different chromosomes-results in shuffled chains of rearrangements ${ }^{47,48}$ (Extended Data Fig. 5a); (2) kataegis, a focal hypermutation process that leads to locally clustered nucleotide substitutions, biased towards a single DNA strand $^{49-51}$ (Extended Data Fig. 5b); and (3) chromothripsis, in which tens to hundreds of DNA breaks occur simultaneously, clustered on one or a few chromosomes, with near-random stitching together of the resulting fragment ${ }^{52-55}$ (Extended Data Fig. $5 \mathrm{c}$ ). We characterized the PCAWG genomes for these three processes (Fig. 4).

Chromoplexy events and reciprocal translocations were identified in 467 (17.8\%) samples (Fig. 4a, c). Chromoplexy was prominent in prostate adenocarcinoma and lymphoid malignancies, as previously described $^{47,48}$, and-unexpectedly-thyroid adenocarcinoma. Different genomic loci were recurrently rearranged by chromoplexy across the three tumour types, mediated by positive selection for particular fusion genes or enhancer-hijacking events. Of 13 fusion genes or enhancer hijacking events in 48 thyroid adenocarcinomas, at least 4 (31\%) were caused by chromoplexy, with a further 4 (31\%) part of complexes that contained chromoplexy footprints (Extended Data Fig. 5a). These events generated fusion genes that involved $R E T$ (two cases) and $N T R K 3$ (one case) ${ }^{56}$, and the juxtaposition of the oncogene IGF2BP3 with regulatory elements from highly expressed genes (five cases).

Kataegis events were found in $60.5 \%$ of all cancers, with particularly high abundance in lung squamous cell carcinoma, bladder cancer, acral melanoma and sarcomas (Fig. 4a, b). Typically, kataegis comprises $\mathrm{C}>\mathrm{N}$ mutations in a $\mathrm{TpC}$ context, which are probably caused by APOBEC activity ${ }^{49-51}$, although a $\mathrm{T}>\mathrm{N}$ conversion in a TpT or CpT process (the affected $\mathrm{T}$ is highlighted in bold) attributed to error-prone polymerases has recently been described ${ }^{57}$. The APOBEC signature accounted for $81.7 \%$ of kataegis events and correlated positively with APOBEC $3 B$ expression levels, somatic SV burden and age at diagnosis (Supplementary Fig. 5). Furthermore, 5.7\% of kataegis events involved the $\mathrm{T}>\mathrm{N}$ error-prone polymerase signature and $2.3 \%$ of events, most notably in sarcomas, showed cytidine deamination in an alternative GpC or CpC context.

Kataegis events were frequently associated with somatic SV breakpoints (Fig. 4a and Supplementary Fig. 6a), as previously described ${ }^{50,51}$. Deletions and complex rearrangements were most-strongly associated with kataegis, whereas tandem duplications and other simple SV classes were only infrequently associated (Supplementary Fig. 6b). Kataegis inducing predominantly $\mathrm{T}>\mathrm{N}$ mutations in CpTpT context was enriched near deletions, specifically those in the 10-25-kilobase (kb) range (Supplementary Fig. 6c).

Samples with extreme kataegis burden (more than 30 foci) comprise four types of focal hypermutation (Extended Data Fig. 6): (1) off-target somatic hypermutation and foci of $\mathrm{T}>\mathrm{N}$ at $\mathrm{CpT} \mathrm{TT}$, found in B cell nonHodgkin lymphoma and oesophageal adenocarcinomas, respectively; (2) APOBEC kataegis associated with complex rearrangements, notably found in sarcoma and melanoma; (3) rearrangement-independent APOBEC kataegis on the lagging strand and in early-replicating regions, mainly found in bladder and head and neck cancer; and (4) a mix of the last two types. Kataegis only occasionally led to driver mutations (Supplementary Table 5).

We identified chromothripsis in 587 samples (22.3\%), most frequently among sarcoma, glioblastoma, lung squamous cell carcinoma, melanoma and breast adenocarcinoma ${ }^{18}$. Chromothripsis increased with whole-genome duplications in most cancer types (Extended Data Fig. 7a), as previously shown in medulloblastoma ${ }^{58}$. The most recurrently associated driver was $T P 53^{52}$ (pan-cancer odds ratio $=3.22$; pan-cancer $P=8.3 \times 10^{-35} ; q<0.05$ in breast lobular (odds ratio $=13$ ), colorectal (odds ratio $=25$ ), prostate (odds ratio $=2.6$ ) and hepatocellular (odds ratio $=3.9$ ) cancers; Fisher-Boschloo tests). In two cancer types (osteosarcoma and B cell lymphoma), women had a higher incidence of chromothripsis than men (Extended Data Fig. 7b). In prostate cancer, we observed a higher incidence of chromothripsis in patients with late-onset than early-onset disease ${ }^{59}$ (Extended Data Fig. 7c).

Chromothripsis regions coincided with $3.6 \%$ of all identified drivers in PCAWG and around 7\% of copy-number drivers (Fig. 4d). These proportions are considerably enriched compared to expectation if selection were not acting on these events (Extended Data Fig. 7d). The majority of coinciding driver events were amplifications (58\%), followed by homozygous deletions (34\%) and SVs within genes or promoter regions ( $8 \%$ ). We frequently observed $\mathrm{a} \geq 2$-fold increase or decrease in expression of amplified or deleted drivers, respectively, when these loci were part of a chromothripsis event, compared with samples without chromothripsis (Extended Data Fig. 7e).

Chromothripsis manifested in diverse patterns and frequencies across tumour types, which we categorized on the basis of five characteristics (Fig. 4a). In liposarcoma, for example, chromothripsis events often involved multiple chromosomes, with universal MDM2 amplification ${ }^{60}$ and co-amplification of TERT in 4 of 19 cases (Fig. $4 \mathrm{~d}$ ). By contrast, in glioblastoma the events tended to affect a smaller region on a single chromosome that was distant from the telomere, resulting in focal amplification of EGFR and MDM2 and loss of CDKN2A. Acral melanomas frequently exhibited $C C N D 1$ amplification, and lung squamous cell carcinomas SOX 2 amplifications. In both cases, these drivers were more-frequently altered by chromothripsis compared with other drivers in the same cancer type and to other cancer types for the same driver (Fig. 4d and Extended Data Fig. 7f). Finally, in chromophobe renal cell carcinoma, chromothripsis nearly always affected chromosome 5(Supplementary Fig. 7): these samples had breakpoints immediately adjacent to TERT, increasing TERT expression by 80 -fold on average compared with samples without rearrangements $(P=0.0004$; MannWhitney $U$-test).

\section{Timing clustered mutations in evolution}

An unanswered question for clustered mutational processes is whether they occur early or late in cancer evolution. To address this, we used molecular clocks to define broad epochs in the life history of each tumour $^{49,61}$. One transition point is between clonal and subclonal mutations: clonal mutations occurred before, and subclonal mutations after, the emergence of the most-recent common ancestor. In regions with copy-number gains, molecular time can be further divided according to whether mutations preceded the copy-number gain (and were themselves duplicated) or occurred after the gain (and therefore present on only one chromosomal copy $)^{7}$.

Chromothripsis tended to have greater relative odds of being clonal than subclonal, suggesting that it occurs early in cancer evolution, especially in liposarcomas, prostate adenocarcinoma and squamous cell lung cancer (Fig. 5a). As previously reported, chromothripsis was especially common in melanomas ${ }^{62}$. We identified 89 separate chromothripsis events that affected 66 melanomas (61\%); 47 out of 89 events affected genes known to be recurrently altered in melanoma ${ }^{63}$ (Supplementary Table 6). Involvement of a region on chromosome 11 that includes the cell-cycle regulator CCND1 occurred in 21 cases (10 out of 86 cutaneous, and 11 out of 21 acral or mucosal melanomas), typically combining chromothripsis with amplification (19 out of 21 cases) (Extended Data Fig. 8). Co-involvement of other cancer-associated genes in the same chromothripsis event was also frequent, including 

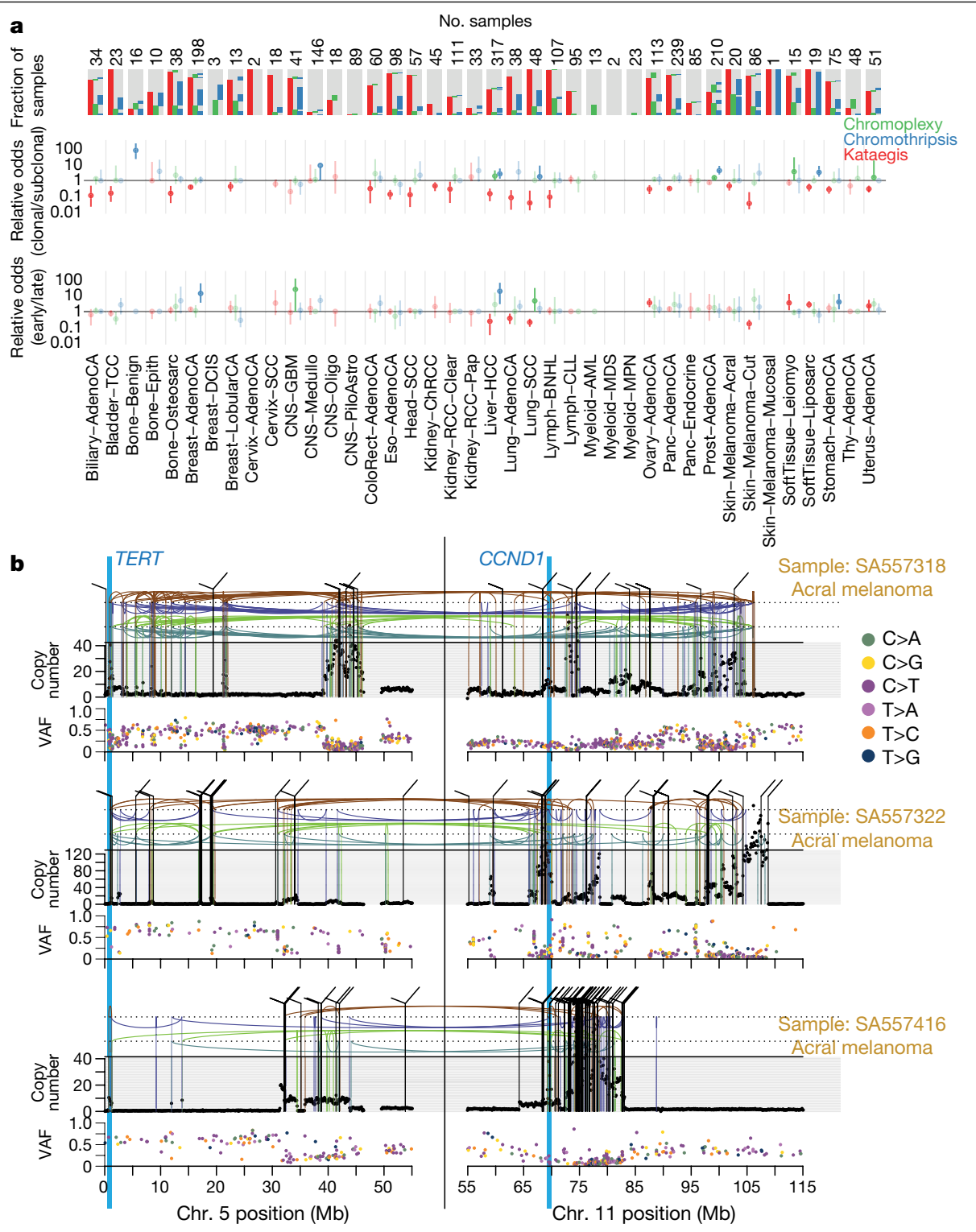

Fig. 5 | Timing of clustered events in PCAWG. a, Extent and timing of chromothripsis, kataegis and chromoplexy across PCAWG. Top, stacked bar charts illustrate co-occurrence of chromothripsis, kataegis and chromoplexy in the samples. Middle, relative odds of clustered events being clonal or subclonal are shown with bootstrapped $95 \%$ confidence intervals. Point estimates are highlighted when they do not overlap odds of 1:1. Bottom, relative odds of the events being early or late clonal are shown as above. Sample sizes (number of patients) are shown across the top. $\mathbf{b}$, Three representative patients with acral melanoma and chromothripsis-induced amplification that simultaneously affects TERT and CCND1. The black points (top) represent sequence coverage from individual genomic bins, with SVs shown as coloured arcs (translocation in black, deletion in purple, duplication in brown, tail-to-tail inversion in cyan and head-to-head inversion in green). Bottom, the variant allele fractions of somatic point mutations.
TERT (five cases), CDKN2A (three cases), TP53 (two cases) and MYC (two cases) (Fig. 5b). In these co-amplifications, a chromothripsis event involving multiple chromosomes initiated the process, creating a derivative chromosome in which hundreds of fragments were stitched together in a near-random order (Fig. 5b). This derivative then rearranged further, leading to massive co-amplification of the multiple target oncogenes together with regions located nearby on the derivative chromosome.

In these cases of amplified chromothripsis, we can use the inferred number of copies bearing each SNV to time the amplification process. SNVs present on the chromosome before amplification will themselves be amplified and are therefore reported in a high fraction of sequence reads (Fig. 5b and Extended Data Fig. 8). By contrast, late SNVs that occur after the amplification has concluded will be present on only one chromosome copy out of many, and thus have a low variant allele fraction. Regions of CCND1 amplification had few-sometimes zero-mutations at high variant allele fraction in acral melanomas, in contrast to later CCND1 amplifications in cutaneous melanomas, in which hundreds to thousands of mutations typically predated amplification (Fig. 5b and Extended Data Fig. 9a, b). Thus, both chromothripsis and the subsequent amplification generally occurred very early during the evolution of acral melanoma. By comparison, in lung squamous cell carcinomas, similar patterns of chromothripsis followed by SOX2 amplification are characterized by many amplified SNVs, suggesting a later event in the evolution of these cancers (Extended Data Fig. 9c).

Notably, in cancer types in which the mutational load was sufficiently high, we could detect a larger-than-expected number of SNVs on an intermediate number of DNA copies, suggesting that they appeared during the amplification process (Supplementary Fig. 8). 

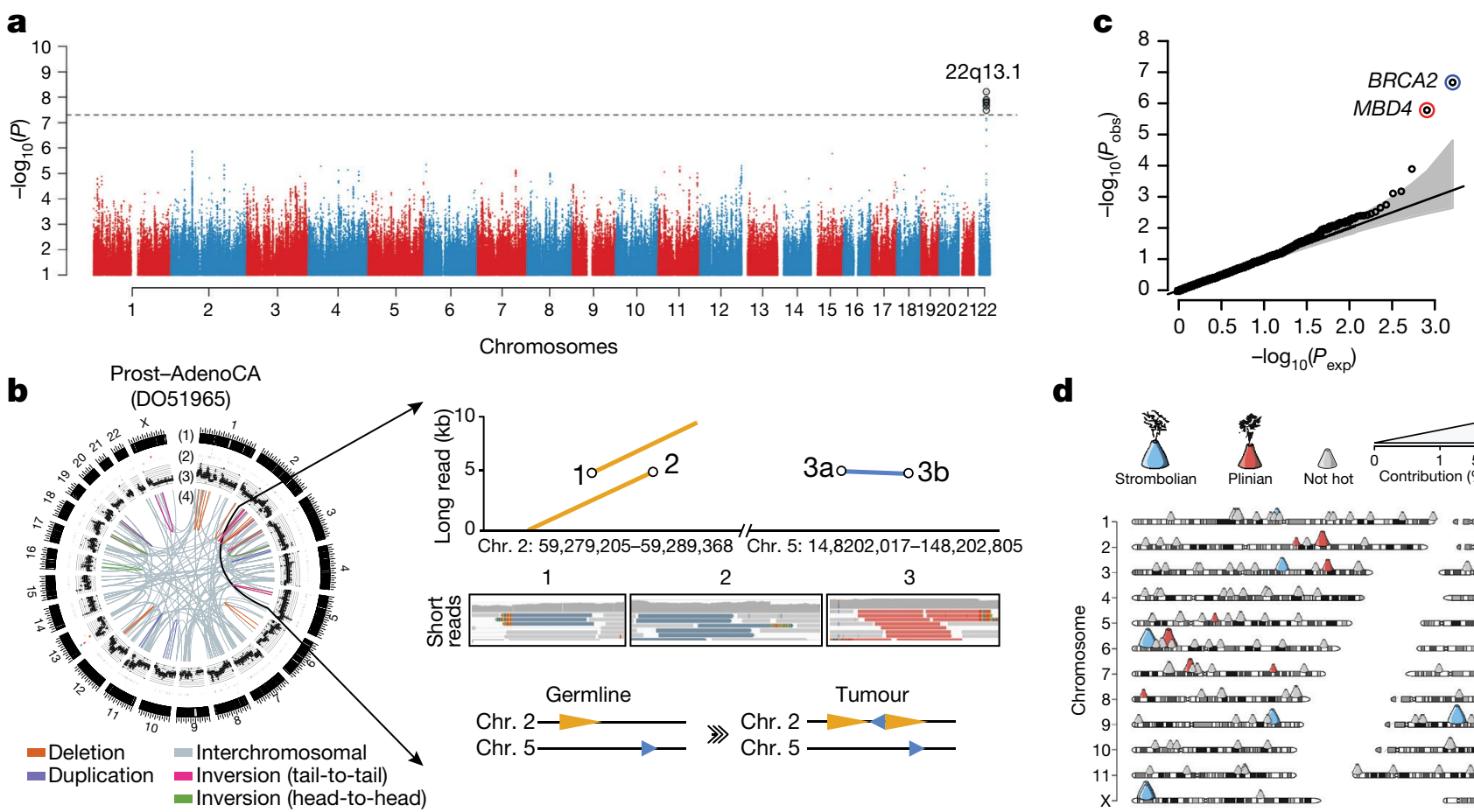

d

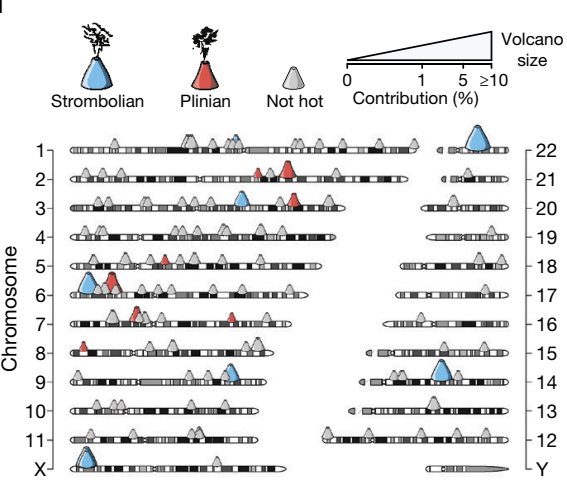

Fig. 6 | Germline determinants of the somatic mutation landscape. a, Association between common ( $\mathrm{MAF}>5 \%$ ) germline variants and somatic APOBEC3B-like mutagenesis in individuals of European ancestry $(n=1,201)$. Two-sided hypothesis testing was performed with PLINK v.1.9. To mitigate multiple-hypothesis testing, the significance threshold was set to genomewide significance $\left(P<5 \times 10^{-8}\right)$. b. Templated insertion SVs in a BRCA1associated prostate cancer. Left, chromosome bands (1);SVs $\leq 10$ megabases (Mb) (2); 1-kb read depth corrected to copy number 0-6 (3); inter- and intrachromosomal SVs $>10 \mathrm{Mb}$ (4). Right, a complex somatic SV composed of a 2.2-kb tandem duplication on chromosome 2 together with a 232-base-pair (bp) inverted templated insertion SV that is derived from chromosome 5 and inserted inbetween the tandem duplication (bottom). Consensus sequence alignment of locally assembled Oxford Nanopore Technologies long sequencing reads to chromosomes 2 and 5 of the human reference genome (top). Breakpoints are circled and marked as 1 (beginning of tandem duplication), 2 (end of tandem duplication) or 3 (inverted templated insertion). For each breakpoint, the middle panel shows Illumina short reads at SV

\section{Germline effects on somatic mutations}

We integrated the set of 88 million germline genetic variant calls with somatic mutations in PCAWG, to study germline determinants of somatic mutation rates and patterns. First, we performed a genomewide association study of somatic mutational processes with common germline variants (minor allele frequency $(\mathrm{MAF})>5 \%$ ) in individuals with inferred European ancestry. An independent genome-wide association study was performed in East Asian individuals from Asian cancer genome projects. We focused on two prevalent endogenous mutational processes: spontaneous deamination of 5-methylcytosine at CpG dinucleotides ${ }^{5}$ (signature 1 ) and activity of the APOBEC 3 family of cytidine deaminases ${ }^{64}$ (signatures 2 and 13 ). No locus reached genomewide significance $\left(P<5 \times 10^{-8}\right)$ for signature 1 (Extended Data Fig. 10a, b). However, a locus at 22q13.1 predicted an APOBEC3B-like mutagenesis at the pan-cancer level ${ }^{65}$ (Fig. 6a). The strongest signal at 22q13.1 was driven by rs12628403, and the minor (non-reference) allele was protective against APOBEC3B-like mutagenesis $\left(\beta=-0.43, P=5.6 \times 10^{-9}\right.$, $\mathrm{MAF}=8.2 \%, n=1,201$ donors) (Extended Data Fig. 10c). This variant tags a common, approximately $30-\mathrm{kb}$ germline SV that deletes the $A P O B E C 3 B$ coding sequence and fuses the $A P O B E C 3 B 3^{\prime}$ untranslated region with the coding sequence of $A P O B E C 3 A$. The deletion is known breakpoints. c, Association between rare germline PTVs (MAF $<0.5 \%$ ) and somatic CpG mutagenesis (approximately with signature 1) in individuals of European ancestry $(n=1,201)$. Genes highlighted in blue or red were associated with lower or higher somatic mutation rates. Two-sided hypothesis testing was performed using linear-regression models with sex, age at diagnosis and cancer project as variables. To mitigate multiple-hypothesis testing, the significance threshold was set to exome-wide significance $\left(P<2.5 \times 10^{-6}\right)$. The black line represents the identity line that would be followed if the observed $P$ values followed the null expectation; the shaded area shows the $95 \%$ confidence intervals. d, Catalogue of polymorphic germline L1 source elements that are active in cancer. The chromosomal map shows germline source L1 elements as volcano symbols. Each volcano is colour-coded according to the type of source L1 activity. The contribution of each source locus (expressed as a percentage) to the total number of transductions identified in PCAWG tumours is represented as a gradient of volcano size, with top contributing elements exhibiting larger sizes.

to increase breast cancer risk and APOBEC mutagenesis in breast cancer genomes $^{66,67}$. Here, we found that rs12628403 reduces APOBEC3Blike mutagenesis specifically in cancer types with low levels of APOBEC mutagenesis $\left(\beta_{\text {low }}=-0.50, P_{\text {low }}=1 \times 10^{-8} ; \beta_{\text {high }}=0.17, P_{\text {high }}=0.2\right)$, and increases APOBEC $3 \mathrm{~A}$-like mutagenesis in cancer types with high levels of APOBEC mutagenesis $\left(\beta_{\text {high }}=0.44, P_{\text {high }}=8 \times 10^{-4} ; \beta_{\text {low }}=-0.21\right.$, $\left.P_{\text {low }}=0.02\right)$. Moreover, we identified a second, novel locus at $22 q 13.1$ that was associated with $\mathrm{APOBEC} 3 \mathrm{~B}$-like mutagenesis across cancer types ( $r$ 2142833, $\left.\beta=0.23, P=1.3 \times 10^{-8}\right)$. We independently validated the association between both loci and APOBEC3B-like mutagenesis using East Asian individuals from Asian cancer genome projects $\left(\beta_{\mathrm{rs} 12628403}=0.57, P_{\mathrm{rs} 12628403}=4.2 \times 10^{-12} ; \beta_{\mathrm{rs} 2142833}=0.58, P_{\mathrm{rs} 2142833}=8 \times 10^{-15}\right)$ (Extended Data Fig. 10d). Notably, in a conditional analysis that accounted for rs12628403, we found that rs2142833 and rs12628403 are inherited independently in Europeans $\left(r^{2}<0.1\right)$, and rs2142833 remained significantly associated with APOBEC3B-like mutagenesis in Europeans $\left(\beta_{\mathrm{EUR}}=0.17, P_{\mathrm{EUR}}=3 \times 10^{-5}\right)$ and East Asians $\left(\beta_{\mathrm{ASN}}=0.25\right.$, $\left.P_{\mathrm{ASN}}=2 \times 10^{-3}\right)$ (Extended Data Fig. 10e, f). Analysis of donor-matched expression data further suggests that rs2142833 is a cis-expression quantitative trait locus (eQTL) for $A P O B E C 3 B$ at the pan-cancer level $\left(\beta=0.19, P=2 \times 10^{-6}\right)$ (Extended Data Fig. 10g, h), consistent with cis-eQTL studies in normal cells ${ }^{68,69}$. 

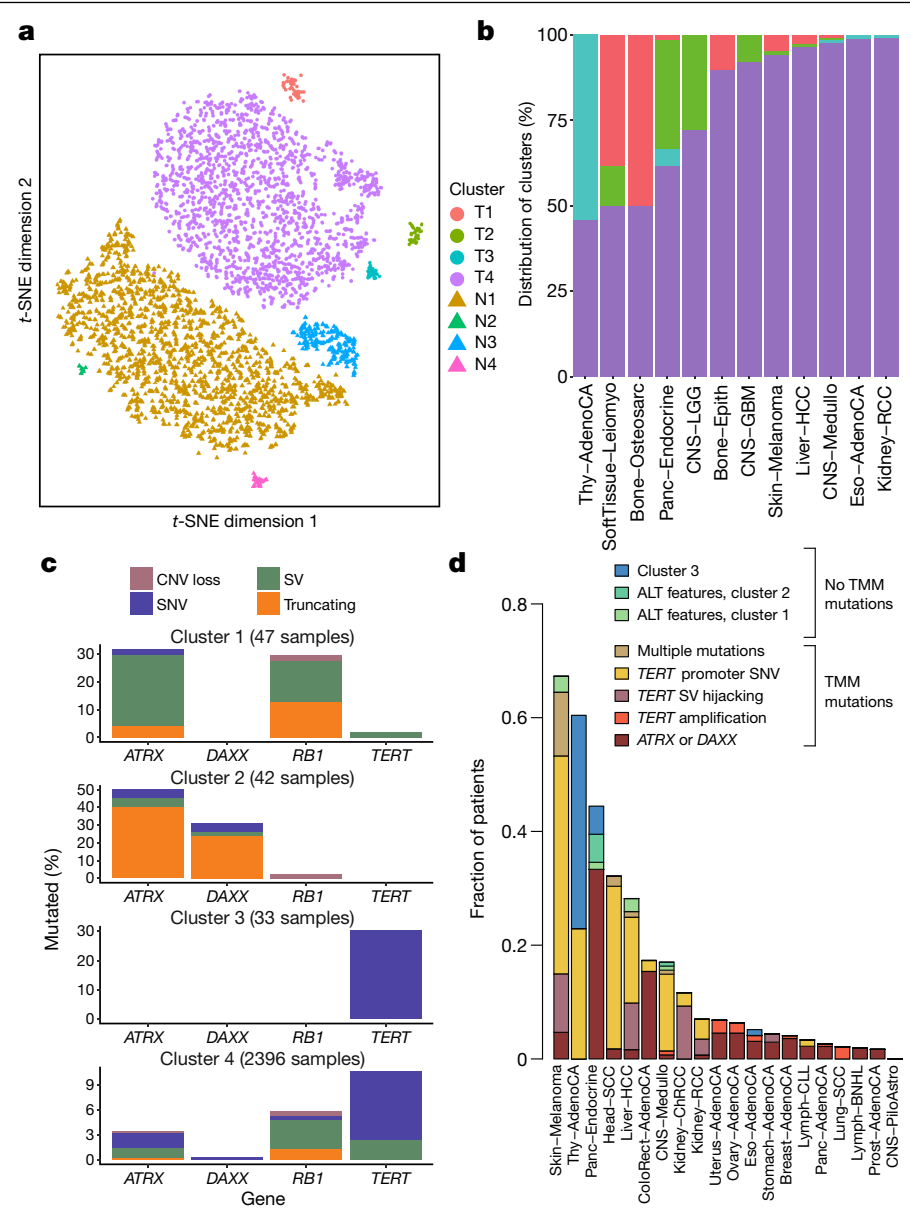

Fig. $7 \mid$ Telomere sequence patterns across PCAWG. a, Scatter plot of the clusters of telomere patterns identified across PCAWG using $t$-distributed stochastic neighbour embedding ( $t$-SNE), based on $n=2,518$ tumour samples and their matched normal samples. Axes have arbitrary dimensions such that samples with similar telomere profiles are clustered together and samples with dissimilar telomere profiles are far apart with high probability. b. Distribution of the four tumour-specific clusters of telomere patterns in selected tumour types from PCAWG.c, Distribution of relevant driver mutations associated with alternative lengthening of telomere and normal telomere maintenance across the four clusters. d, Distribution of telomere maintenance abnormalities across tumour types with more than 40 patients in PCAWG. Samples were classified as tumour clusters 1-3 if they fell into a relevant cluster without mutations in TERT, ATRX or DAXX and had no ALT phenotype. TMM, telomere maintenance mechanisms.

Second, we performed a rare-variant association study $(\mathrm{MAF}<0.5 \%)$ to investigate the relationship between germline PTVs and somatic DNA rearrangements in individuals with European ancestry (Extended Data Fig. 11a-c). Germline BRCA2 and BRCA1 PTVs were associated with an increased burden of small (less than $10 \mathrm{~kb}$ ) somatic SV deletions $\left(P=1 \times 10^{-8}\right)$ and tandem duplications $\left(P=6 \times 10^{-13}\right)$, respectively, corroborating recent studies in breast and ovarian cancer $^{30,70}$. In PCAWG data, this pattern also extends to other tumour types, including adenocarcinomas of the prostate and pancreas ${ }^{6}$, typically in the setting of biallelic inactivation. In addition, tumours with high levels of small SV tandem duplications frequently exhibited a novel and distinct class of SVs termed 'cycles of templated insertions' ${ }^{\text {t }}$. These complex SV events consist of DNA templates that are copied from across the genome, joined into one contiguous sequence and inserted into a single derivative chromosome. We found a significant association between germline $B R C A 1$ PTVs and templated insertions at the pan-cancer level $\left(P=4 \times 10^{-15}\right)$ (Extended Data Fig. 11d, e). Whole-genome long-read sequencing data generated for a BRCA1-deficient PCAWG prostate tumour verified the small tandem-duplication and templatedinsertion SV phenotypes (Fig. 6b). Almost all (20 out of 21) of BRCA1associated tumours with a templated-insertion SV phenotype displayed combined germline and somatic hits in the gene. Together, these data suggest that biallelic inactivation of $B R C A 1$ is a driver of the templatedinsertion SV phenotype.

Third, rare-variant association analysis revealed that patients with germline MBD4 PTVs had increased rates of somatic $\mathrm{C}>\mathrm{T}$ mutation rates at $\mathrm{CpG}$ dinucleotides $\left(P<2.5 \times 10^{-6}\right)$ (Fig. $6 \mathrm{c}$ and Extended Data Fig. 11f, g). Analysis of previously published whole-exome sequencing samples from the TCGA $(n=8,134)$ replicated the association between germline $M B D 4$ PTVs and increased somatic $\mathrm{CpG}$ mutagenesis at the pan-cancer level $\left(P=7.1 \times 10^{-4}\right)$ (Extended Data Fig. 11h). Moreover, gene-expression profiling revealed a significant but modest correlation between $M B D 4$ expression and somatic $\mathrm{CpG}$ mutation rates between and within PCAWG tumour types (Extended Data Fig. 11i-k). MBD4 encodes a DNA-repair gene that removes thymidines from T:G mismatches within methylated $\mathrm{CpG}$ sites $^{71}$, a functionality that would be consistent with a $\mathrm{CpG}$ mutational signature in cancer.

Fourth, we assessed long interspersed nuclear elements (LINE-1; L1 hereafter) that mediate somatic retrotransposition events ${ }^{72-74}$. We identified 114 germline source L1 elements capable of active somatic retrotransposition, including 70 that represent insertions with respect to the human reference genome (Fig. 6d and Supplementary Table 7), and 53 that were tagged by single-nucleotide polymorphisms in strong linkage disequilibrium (Supplementary Table 7). Only 16 germline L1 elements accounted for $67 \%(2,440$ out of 3,669$)$ of all L1-mediated transductions ${ }^{10}$ detected in the PCAWG dataset (Extended Data Fig. 12a). These 16 hot-L1 elements followed two broad patterns of somatic activity (8 of each), which we term Strombolian and Plinian in analogy to patterns of volcanic activity. Strombolian L1s are frequently active in cancer, but mediate only small-to-modest eruptions of somatic L1 activity in cancer samples (Extended Data Fig. 12b). By contrast, Plinian L1s are more rarely seen, but display aggressive somatic activity. Whereas Strombolian elements are typically relatively common (MAF $>2 \%$ ) and sometimes even fixed in the human population, all Plinian elements were infrequent $(\mathrm{MAF} \leq 2 \%)$ in PCAWG donors (Extended Data Fig. 12c; $P=0.001$, Mann-Whitney $U$-test). This dichotomous pattern of activity and allele frequency may reflect differences in age and selective pressures, with Plinian elements potentially inserted into the human germline more recently. PCAWG donors bear on average between 50 and $60 \mathrm{~L} 1$ source elements and between 5 and 7 elements with hot activity (Extended Data Fig. 12d), but only 38\% $(1,075$ out of 2,814$)$ of PCAWG donors carried $\geq 1$ Plinian element. Some L1 germline source loci caused somatic loss of tumour-suppressor genes (Extended Data Fig. 12e). Many are restricted to individual continental population ancestries (Extended Data Fig. 12f-j).

\section{Replicative immortality}

One of the hallmarks of cancer is the ability of cancer to evade cellular senescence ${ }^{21}$. Normal somatic cells typically have finite cell division potential; telomere attrition is one mechanism to limit numbers of mitoses $^{75}$. Cancers enlist multiple strategies to achieve replicative immortality. Overexpression of the telomerase gene, TERT, which maintains telomere lengths, is especially prevalent. This can be achieved through point mutations in the promoter that lead to de novo transcription factor binding ${ }^{34,37}$; hitching $T E R T$ to highly active regulatory elements elsewhere in the genome ${ }^{46,76}$; insertions of viral enhancers upstream of the gene $\mathrm{e}^{77,78}$; and increased dosage through chromosomal amplification, as we have seen in melanoma (Fig. 5b). In addition, there is an 'alternative lengthening of telomeres' (ALT) pathway, in which telomeres are lengthened through homologous recombination, mediated by loss-of-function mutations in the ATRX and DAXX genes $^{79}$. 
As reported in a companion paper ${ }^{13}, 16 \%$ of tumours in the PCAWG dataset exhibited somatic mutations in at least one of $A T R X, D A X X$ and TERT. TERT alterations were detected in 270 samples, whereas 128 tumours had alterations in $A T R X$ or $D A X X$, of which 71 were proteintruncating. In the companion paper, which focused on describing patterns of ALT and TERT-mediated telomere maintenance ${ }^{13}, 12$ features of telomeric sequence were measured in the PCAWG cohort. These included counts of nine variants of the core hexameric sequence, the number of ectopic telomere-like insertions within the genome, the number of genomic breakpoints and telomere length as a ratio between tumour and normal. Here we used the 12 features as an overview of telomere integrity across all tumours in the PCAWG dataset.

On the basis of these 12 features, tumour samples formed 4 distinct subclusters (Fig. 7a and Extended Data Fig. 13a), suggesting that telomere-maintenance mechanisms are more diverse than the wellestablished TERT and ALT dichotomy. Clusters C1 (47 tumours) and C2 (42 tumours) were enriched for traits of the ALT pathway-having longer telomeres, more genomic breakpoints, more ectopic telomere insertions and variant telomere sequence motifs (Supplementary Fig. 9). $\mathrm{C} 1$ and $\mathrm{C} 2$ were distinguished from one another by the latter having a considerable increase in the number of TTCGGG and TGAGGG variant motifs among the telomeric hexamers. Thyroid adenocarcinomas were markedly enriched among C3 samples ( 26 out of $33 \mathrm{C} 3$ samples; $P<10^{-16}$ ); the $C 1$ cluster (ALT subtype 1 ) was common among sarcomas; and both pancreatic endocrine neoplasms and low-grade gliomas had a high proportion of samples in the $\mathrm{C} 2$ cluster (ALT subtype 2) (Fig. 7b). Notably, some of the thyroid adenocarcinomas and pancreatic neuroendocrine tumours that cluster together (cluster C3) had matched normal samples that also cluster together (normal cluster N3) (Extended Data Fig. 13a) and which share common properties. For example, the GTAGGG repeat was overrepresented among samples in this group (Supplementary Fig. 10).

Somatic driver mutations were also unevenly distributed across the four clusters (Fig. $7 \mathrm{c}$ ). C1 tumours were enriched for $R B 1$ mutations or SVs $\left(P=3 \times 10^{-5}\right)$, as well as frequent SVs that affected $A T R X\left(P=6 \times 10^{-14}\right)$, but not $D A X X$. RB1 and $A T R X$ mutations were largely mutually exclusive (Extended Data Fig. 13b). By contrast, C2 tumours were enriched for somatic point mutations in $A T R X$ and $D A X X\left(P=6 \times 10^{-5}\right)$, but not $R B 1$. The enrichment of $R B 1$ mutations in $\mathrm{C} 1$ remained significant when only leiomyosarcomas and osteosarcomas were considered, confirming that this enrichment is not merely a consequence of the different distribution of tumour types across clusters. C3 samples had frequent TERT promoter mutations $\left(30 \% ; P=2 \times 10^{-6}\right)$.

There was a marked predominance of $R B 1$ mutations in $\mathrm{C} 1$. Nearly a third of the samples in $\mathrm{C} 1$ contained an $R B 1$ alteration, which were evenly distributed across truncating SNVs, SVs and shallow deletions (Extended Data Fig. 13c). Previous research has shown that $R B 1$ mutations are associated with long telomeres in the absence of TERT mutations and $A T R X$ inactivation ${ }^{80}$, and studies using mouse models have shown that knockout of $\mathrm{Rb}$-family proteins causes elongated telomeres $^{81}$. The association with the $\mathrm{C} 1$ cluster here suggests that $R B 1$ mutations can represent another route to activating the ALT pathway, which has subtly different properties of telomeric sequence compared with the inactivation of $D A X X$-these fall almost exclusively in cluster C2.

Tumour types with the highest rates of abnormal telomere maintenance mechanisms often originate in tissues that have low endogenous replicative activity (Fig. 7d). In support of this, we found an inverse correlation between previously estimated rates of stem cell division across tissues $^{82}$ and the frequency of telomere maintenance abnormalities $(P=0.01$, Poisson regression) (Extended Data Fig. 13d). This suggests that restriction of telomere maintenance is an important tumoursuppression mechanism, particularly in tissues with low steady-state cellular proliferation, in which a clone must overcome this constraint to achieve replicative immortality.

\section{Conclusions and future perspectives}

The resource reported in this paper and its companion papers has yielded insights into the nature and timing of the many mutational processes that shape large- and small-scale somatic variation in the cancer genome; the patterns of selection that act on these variations; the widespread effect of somatic variants on transcription; the complementary roles of the coding and non-coding genome for both germline and somatic mutations; the ubiquity of intratumoral heterogeneity; and the distinctive evolutionary trajectory of each cancer type. Many of these insights can be obtained only from an integrated analysis of all classes of somatic mutation on a wholegenome scale, and would not be accessible with, for example, targeted exome sequencing.

The promise of precision medicine is to match patients to targeted therapies using genomics. A major barrier to its evidence-based implementation is the daunting heterogeneity of cancer chronicled in these papers, from tumour type to tumour type, from patient to patient, from clone to clone and from cell to cell. Building meaningful clinical predictors from genomic data can be achieved, but will require knowledge banks comprising tens of thousands of patients with comprehensive clinical characterization ${ }^{83}$. As these sample sizes will be too large for any single funding agency, pharmaceutical company or health system, international collaboration and data sharing will be required. The next phase of ICGC, ICGC-ARGO (https:// www.icgc-argo.org/), will bring the cancer genomics community together with healthcare providers, pharmaceutical companies, data science and clinical trials groups to build comprehensive knowledge banks of clinical outcome and treatment data from patients with a wide variety of cancers, matched with detailed molecular profiling.

Extending the story begun by TCGA, ICGC and other cancer genomics projects, the PCAWG has brought us closer to a comprehensive narrative of the causal biological changes that drive cancer phenotypes. We must now translate this knowledge into sustainable, meaningful clinical treatments.

\section{Online content}

Any methods, additional references, Nature Research reporting summaries, source data, extended data, supplementary information, acknowledgements, peer review information; details of author contributions and competing interests; and statements of data and code availability are available at https://doi.org/10.1038/s41586-020-1969-6.

1. Pleasance, E. D. et al. A comprehensive catalogue of somatic mutations from a human cancer genome. Nature 463, 191-196 (2010).

2. Pleasance, E. D. et al. A small-cell lung cancer genome with complex signatures of tobacco exposure. Nature 463, 184-190 (2010).

3. Ley, T. J. et al. DNA sequencing of a cytogenetically normal acute myeloid leukaemia genome. Nature 456, 66-72 (2008).

4. Rheinbay, E. et al. Analyses of non-coding somatic drivers in 2,693 cancer whole genomes. Nature https://doi.org/10.1038/s41586-020-1965-x (2020).

5. Alexandrov, L. B. et al. The repertoire of mutational signatures in human cancer. Nature https://doi.org/10.1038/s41586-020-1943-3 (2020).

6. Li, Y. et al. Patterns of somatic structural variation in human cancer genomes. Nature https://doi.org/10.1038/s41586-019-1913-9 (2020).

7. Gerstung, M. et al. The evolutionary history of 2,658 cancers. Nature https://doi.org/ 10.1038/s41586-019-1907-7 (2020)

8. PCAWG Transcriptome Core Group et al. Genomic basis of RNA alterations in cancer. Nature https://doi.org/10.1038/s41586-020-1970-0 (2020).

9. Zhang, Y. et al. High-coverage whole-genome analysis of 1,220 cancers reveals hundreds of genes deregulated by rearrangement-mediated cis-regulatory alterations. Nat. Commun. https://doi.org/10.1038/s41467-019-13885-w (2020).

10. Rodriguez-Martin, B. et al. Pan-cancer analysis of whole genomes identifies driver rearrangements promoted by LINE-1 retrotransposition. Nat. Genet. https://doi. org/10.1038/s41588-019-0562-0 (2020).

11. Zapatka, M. et al. The landscape of viral associations in human cancers. Nat. Genet. https://doi.org/10.1038/s41588-019-0558-9 (2020).

12. Jiao, W. et al. A deep learning system can accurately classify primary and metastatic cancers based on patterns of passenger mutations. Nat. Commun. https://doi.org/ 10.1038/s41467-019-13825-8 (2020). 
13. Sieverling, L. et al. Genomic footprints of activated telomere maintenance mechanisms in cancer. Nat. Commun. https://doi.org/10.1038/s41467-019-13824-9 (2020).

14. Yuan, Y. et al. Comprehensive molecular characterization of mitochondrial genomes in human cancers. Nat. Genet. https://doi.org/10.1038/s41588-019-0557-x (2020).

15. Akdemir, K. C. et al. Chromatin folding domains disruptions by somatic genomic rearrangements in human cancers. Nat. Genet. https://doi.org/10.1038/s41588-0190564-y (2020).

16. Reyna, M. A. et al. Pathway and network analysis of more than 2,500 whole cancer genomes. Nat. Commun. https://doi.org/10.1038/s41467-020-14351-8 (2020).

17. Bailey, M. H. et al. Retrospective evaluation of whole exome and genome mutation calls in 746 cancer samples. Nat. Commun. (2020).

18. Cortes-Ciriano, I. et al. Comprehensive analysis of chromothripsis in 2,658 human cancers using whole-genome sequencing. Nat. Genet. https://doi.org/10.1038/s41588019-0576-7 (2020).

19. Bray, F., Ren, J.-S., Masuyer, E. \& Ferlay, J. Global estimates of cancer prevalence for 27 sites in the adult population in 2008. Int. J. Cancer 132, 1133-1145 (2013).

20. Tarver, T. Cancer Facts \& Figures 2012. American Cancer Society (ACS). J. Consum. Health Internet 16, 366-367 (2012).

21. Hanahan, D. \& Weinberg, R. A. Hallmarks of cancer: the next generation. Cell 144, 646-674 (2011).

22. International Cancer Genome Consortium. International network of cancer genome projects. Nature 464, 993-998 (2010).

23. Bailey, M. H. et al. Comprehensive characterization of cancer driver genes and mutations. Cell 173, 371-385 (2018).

24. Sanchez-Vega, F. et al. Oncogenic signaling pathways in The Cancer Genome Atlas. Cell 173, 321-337 (2018).

25. Hoadley, K. A. et al. Cell-of-origin patterns dominate the molecular classification of 10,000 tumors from 33 types of cancer. Cell 173, 291-304 (2018).

26. Stein, L. D., Knoppers, B. M., Campbell, P., Getz, G. \& Korbel, J. O. Data analysis: create a cloud commons. Nature 523, 149-151 (2015).

27. Phillips, M. et al. Genomics: data sharing needs international code of conduct. Nature https://doi.org/10.1038/d41586-020-00082-9 (2020).

28. Krochmalski, J. Developing with Docker (Packt Publishing, 2016).

29. Welch, J. S. et al. The origin and evolution of mutations in acute myeloid leukemia. Cell 150, 264-278 (2012).

30. Nik-Zainal, S. et al. Landscape of somatic mutations in 560 breast cancer whole-genome sequences. Nature 534, 47-54 (2016)

31. Meier, B. et al. C. elegans whole-genome sequencing reveals mutational signatures related to carcinogens and DNA repair deficiency. Genome Res. 24, 1624-1636 (2014).

32. Martincorena, I. et al. Universal patterns of selection in cancer and somatic tissues. Cell 171, 1029-1041 (2017).

33. Tamborero, D. et al. Cancer Genome Interpreter annotates the biological and clinical relevance of tumor alterations. Genome Med. 10, 25 (2018).

34. Huang, F. W. et al. Highly recurrent TERT promoter mutations in human melanoma. Science 339, 957-959 (2013).

35. Rheinbay, E. et al. Recurrent and functional regulatory mutations in breast cancer. Nature 547, 55-60 (2017).

36. Fredriksson, N. J., Ny, L., Nilsson, J. A. \& Larsson, E. Systematic analysis of noncoding somatic mutations and gene expression alterations across 14 tumor types. Nat. Genet. 46, 1258-1263 (2014).

37. Horn, S. et al. TERT promoter mutations in familial and sporadic melanoma. Science $\mathbf{3 3 9}$ 959-961 (2013).

38. Ciriello, G. et al. Emerging landscape of oncogenic signatures across human cancers. Nat. Genet. 45, 1127-1133 (2013).

39. Rahman, N. Realizing the promise of cancer predisposition genes. Nature 505, 302-308 (2014).

40. Pearl, L. H., Schierz, A. C., Ward, S. E., Al-Lazikani, B. \& Pearl, F. M. G. Therapeutic opportunities within the DNA damage response. Nat. Rev. Cancer 15, 166-180 (2015).

41. Taylor-Weiner, A. et al. DeTiN: overcoming tumor-in-normal contamination. Nat. Methods 15, 531-534 (2018)

42. Fujimoto, A. et al. Whole-genome mutational landscape and characterization of noncoding and structural mutations in liver cancer. Nat. Genet. 48, 500-509 (2016).

43. Shlush, L. I. Age-related clonal hematopoiesis. Blood 131, 496-504 (2018).

44. Northcott, P. A. et al. The whole-genome landscape of medulloblastoma subtypes. Nature 547, 311-317 (2017).

45. Scarpa, A. et al. Whole-genome landscape of pancreatic neuroendocrine tumours. Nature 543, 65-71 (2017).

46. Davis, C. F. et al. The somatic genomic landscape of chromophobe renal cell carcinoma. Cancer Cell 26, 319-330 (2014).

47. Berger, M. F. et al. The genomic complexity of primary human prostate cancer. Nature 470, 214-220 (2011).

48. Baca, S. C. et al. Punctuated evolution of prostate cancer genomes. Cell 153, 666-677 (2013)

49. Nik-Zainal, S. et al. The life history of 21 breast cancers. Cell 149, 994-1007 (2012)

50. Nik-Zainal, S. et al. Mutational processes molding the genomes of 21 breast cancers. Cell 149, 979-993 (2012)

51. Roberts, S. A. et al. Clustered mutations in yeast and in human cancers can arise from damaged long single-strand DNA regions. Mol. Cell 46, 424-435 (2012).

52. Rausch, T. et al. Genome sequencing of pediatric medulloblastoma links catastrophic DNA rearrangements with TP53 mutations. Cell 148, 59-71 (2012).

53. Stephens, P. J. et al. Massive genomic rearrangement acquired in a single catastrophic event during cancer development. Cell 144, 27-40 (2011).

54. Korbel, J. O. \& Campbell, P. J. Criteria for inference of chromothripsis in cancer genomes. Cell 152, 1226-1236 (2013).

55. Zhang, C.-Z. et al. Chromothripsis from DNA damage in micronuclei. Nature 522, 179-184 (2015).

56. The Cancer Genome Atlas Research Network. Integrated genomic characterization of papillary thyroid carcinoma. Cell 159, 676-690 (2014).
57. Supek, F. \& Lehner, B. Clustered mutation signatures reveal that error-prone DNA repair targets mutations to active genes. Cell 170, 534-547 (2017).

58. Mardin, B. R. et al. A cell-based model system links chromothripsis with hyperploidy. Mol. Syst. Biol. 11, 828 (2015).

59. Weischenfeldt, J. et al. Integrative genomic analyses reveal an androgen-driven somatic alteration landscape in early-onset prostate cancer. Cancer Cell 23, 159-170 (2013).

60. Garsed, D. W. et al. The architecture and evolution of cancer neochromosomes. Cancer Cell 26, 653-667 (2014).

61. Durinck, S. et al. Temporal dissection of tumorigenesis in primary cancers. Cancer Discov. 1, 137-143 (2011).

62. Hayward, N. K. et al. Whole-genome landscapes of major melanoma subtypes. Nature 545, 175-180 (2017).

63. The Cancer Genome Atlas Network. Genomic classification of cutaneous melanoma. Cell 161, 1681-1696 (2015).

64. Alexandrov, L. B. et al. Signatures of mutational processes in human cancer. Nature $\mathbf{5 0 0}$ 415-421 (2013).

65. Chan, K. et al. An APOBEC3A hypermutation signature is distinguishable from the signature of background mutagenesis by APOBEC3B in human cancers. Nat. Genet. 47, 1067-1072 (2015).

66. Nik-Zainal, S. et al. Association of a germline copy number polymorphism of APOBEC3A and $A P O B E C 3 B$ with burden of putative $A P O B E C$-dependent mutations in breast cancer. Nat. Genet. 46, 487-491 (2014).

67. Middlebrooks, C. D. et al. Association of germline variants in the APOBEC3 region with cancer risk and enrichment with APOBEC-signature mutations in tumors. Nat. Genet. 48 , 1330-1338 (2016)

68. Westra, H.-J. et al. Systematic identification of trans eQTLs as putative drivers of known disease associations. Nat. Genet. 45, 1238-1243 (2013).

69. Stranger, B. E. et al. Population genomics of human gene expression. Nat. Genet. 39, 1217-1224 (2007).

70. Menghi, F. et al. The tandem duplicator phenotype as a distinct genomic configuration in cancer. Proc. Natl Acad. Sci. USA 113, E2373-E2382 (2016).

71. Hendrich, B., Hardeland, U., Ng, H. H., Jiricny, J. \& Bird, A. The thymine glycosylase MBD4 can bind to the product of deamination at methylated $\mathrm{CpG}$ sites. Nature $401,301-304$ (1999).

72. Lee, E. et al. Landscape of somatic retrotransposition in human cancers. Science $\mathbf{3 3 7}$ 967-971 (2012).

73. Tubio, J. M. C. et al. Extensive transduction of nonrepetitive DNA mediated by L1 retrotransposition in cancer genomes. Science 345, 1251343-1251343 (2014).

74. Helman, E. et al. Somatic retrotransposition in human cancer revealed by whole-genome and exome sequencing. Genome Res. 24, 1053-1063 (2014).

75. Shay, J. W. \& Wright, W. E. Hayflick, his limit, and cellular ageing. Nat. Rev. Mol. Cell Biol. 1, 72-76 (2000).

76. Peifer, M. et al. Telomerase activation by genomic rearrangements in high-risk neuroblastoma. Nature 526, 700-704 (2015).

77. Totoki, Y. et al. Trans-ancestry mutational landscape of hepatocellular carcinoma genomes. Nat. Genet. 46, 1267-1273 (2014).

78. Paterlini-Bréchot, P. et al. Hepatitis B virus-related insertional mutagenesis occurs frequently in human liver cancers and recurrently targets human telomerase gene. Oncogene 22, 3911-3916 (2003).

79. Heaphy, C. M. et al. Prevalence of the alternative lengthening of telomeres telomere maintenance mechanism in human cancer subtypes. Am. J. Pathol. 179, 1608-1615 (2011).

80. Barthel, F. P. et al. Systematic analysis of telomere length and somatic alterations in 31 cancer types. Nat. Genet. 49, 349-357 (2017).

81. García-Cao, M., Gonzalo, S., Dean, D. \& Blasco, M. A. A role for the Rb family of proteins in controlling telomere length. Nat. Genet. 32, 415-419 (2002).

82. Tomasetti, C. \& Vogelstein, B. Variation in cancer risk among tissues can be explained by the number of stem cell divisions. Science 347, 78-81 (2015).

83. Gerstung, M. et al. Precision oncology for acute myeloid leukemia using a knowledge bank approach. Nat. Genet. 49, 332-340 (2017).

84. O'Connor, B. D. et al. The Dockstore: enabling modular, community-focused sharing of Docker-based genomics tools and workflows. F1000Res. 6, 52 (2017)

85. Zhang, J. et al. The International Cancer Genome Consortium Data Portal. Nat. Biotechnol. 37, 367-369 (2019).

86. Miller, C. A., Qiao, Y., DiSera, T., D'Astous, B. \& Marth, G. T. bam.iobio: a web-based, real-time, sequence alignment file inspector. Nat. Methods 11, 1189-1189 (2014).

87. Goldman, M. et al. The UCSC Xena platform for public and private cancer genomics data visualization and interpretation. Preprint at https://www.biorxiv.org/content/10.1101/ 326470v6 (2019).

88. Papatheodorou, I. et al. Expression Atlas: gene and protein expression across multiple studies and organisms. Nucleic Acids Res. 46, D246-D251 (2018).

Publisher's note Springer Nature remains neutral with regard to jurisdictional claims in published maps and institutional affiliations.

Open Access This article is licensed under a Creative Commons Attribution 4.0 International License, which permits use, sharing, adaptation, distribution and reproduction in any medium or format, as long as you give appropriate redit to the original author(s) and the source, provide a link to the Creative Commons license, and indicate if changes were made. The images or other third party material in this article are included in the article's Creative Commons license, unless indicated otherwise in a credit line to the material. If material is not included in the article's Creative Commons license and your intended use is not permitted by statutory regulation or exceeds the permitted use, you will need to obtain permission directly from the copyright holder. To view a copy of this license, visit http://creativecommons.org/licenses/by/4.0/.

(c) The Author(s) 2020, corrected publication 2023 
The ICGC/TCGA Pan-Cancer Analysis of Whole Genomes Consortium

Lauri A. Aaltonen', Federico Abascal ${ }^{2}$, Adam Abeshouse ${ }^{3}$, Hiroyuki Aburatani ${ }^{4}$, David J. Adams ${ }^{2}$, Nishant Agrawal ${ }^{5}$, Keun Soo Ahn ${ }^{6}$, Sung-Min Ahn ${ }^{7}$, Hiroshi Aikata ${ }^{8}$, Rehan Akbani ${ }^{9}$, Kadir C. Akdemir ${ }^{10}$, Hikmat Al-Ahmadie ${ }^{3}$, Sultan T. Al-Sedairy", Fatima Al-Shahrour ${ }^{12}$, Malik Alawi ${ }^{13,14}$, Monique Albert ${ }^{15}$, Kenneth Aldape ${ }^{16,17}$, Ludmil B. Alexandrov ${ }^{2,18,19}$, Adrian Ally ${ }^{20}$, Kathryn Alsop ${ }^{21}$, Eva G. Alvarez ${ }^{22,23,24}$, Fernanda Amary ${ }^{25}$, Samirkumar B. Amin ${ }^{26,27,28}$, Brice Aminou ${ }^{29}$, Ole Ammerpohl ${ }^{30,31}$, Matthew J. Anderson ${ }^{32}$, Yeng Ang ${ }^{33}$, Davide Antonello ${ }^{34}$, Pavana Anur ${ }^{35}$, Samuel Aparicio ${ }^{36}$ Elizabeth L. Appelbaum ${ }^{37,38}$, Yasuhito Arai ${ }^{39}$, Axel Aretz ${ }^{40}$, Koji Arihiro ${ }^{8}$, Shun-ichi Ariizumi ${ }^{41}$ Joshua Armenia ${ }^{42}$, Laurent Arnould ${ }^{43}$, Sylvia Asa ${ }^{44,45}$, Yassen Assenov ${ }^{46}$, Gurnit Atwal ${ }^{47,48,49}$, Sietse Aukema ${ }^{31,50}$, J. Todd Auman ${ }^{51}$, Miriam R. R. Aure ${ }^{52}$, Philip Awadalla ${ }^{47,48}$, Marta Aymerich ${ }^{53}$, Gary D. Bader ${ }^{48}$, Adrian Baez-Ortega ${ }^{54}$, Matthew H. Bailey ${ }^{37,55}$, Peter J. Bailey ${ }^{56}$, Miruna Balasundaram ${ }^{20}$, Saianand Balu ${ }^{57}$, Pratiti Bandopadhayay ${ }^{58,59,60}$, Rosamonde E. Banks ${ }^{61}$, Stefano Barbi ${ }^{62}$, Andrew P. Barbour ${ }^{63,64}$, Jonathan Barenboim ${ }^{47}$, Jill Barnholtz-Sloan ${ }^{65,66}$, Hugh Barr ${ }^{67}$, Elisabet Barrera ${ }^{68}$, John Bartlett ${ }^{15,69}$, Javier Bartolome ${ }^{70}$, Claudio Bassi ${ }^{34}$, Oliver F. Bathe ${ }^{71,72}$, Daniel Baumhoer ${ }^{73}$, Prashant Bavi ${ }^{74}$, Stephen B. Baylin ${ }^{75,76}$, Wojciech Bazant ${ }^{68}$, Duncan Beardsmore ${ }^{77}$, Timothy A. Beck ${ }^{78,79}$, Sam Behjati ${ }^{2}$, Andreas Behren ${ }^{80}$, Beifang Niu ${ }^{81}$, Cindy Bell ${ }^{82}$, Sergi Beltran ${ }^{83,84}$, Christopher Benz ${ }^{85}$, Andrew Berchuck ${ }^{86}$, Anke K. Bergmann ${ }^{87}$, Erik N. Bergstrom ${ }^{18,19}$, Benjamin P. Berman ${ }^{88,89,90}$, Daniel M. Berney ${ }^{91}$, Stephan H. Bernhart ${ }^{92,93,94}$, Rameen Beroukhim ${ }^{58,95,96,97}$, Mario Berrios ${ }^{98}$, Samantha Bersani ${ }^{99}$, Johanna Bertl ${ }^{100,101}$ Miguel Betancourt ${ }^{102}$, Vinayak Bhandari ${ }^{47,103}$, Shriram G. Bhosle ${ }^{2}$, Andrew V. Biankin ${ }^{56,104,105,106}$, Matthias Bieg ${ }^{107,108}$, Darell Bigner ${ }^{109}$, Hans Binder ${ }^{92,93}$, Ewan Birney ${ }^{68}$, Michael Birrer ${ }^{110}$, Nidhan K. Biswas ${ }^{111}$, Bodil Bjerkehagen ${ }^{73,12}$, Tom Bodenheimer ${ }^{57}$, Lori Boice ${ }^{113}$, Giada Bonizzato ${ }^{114}$, Johann S. De Bono ${ }^{115}$, Arnoud Boot ${ }^{16,117}$, Moiz S. Bootwalla ${ }^{98}$, Ake Borg ${ }^{118}$, Arndt Borkhardt ${ }^{119}$, Keith A. Boroevich ${ }^{120,121}$, Ivan Borozan ${ }^{47}$, Christoph Borst ${ }^{122}$, Marcus Bosenberg ${ }^{123}$, Mattia Bosio ${ }^{70,84,124}$, Jacqueline Boultwood ${ }^{125}$, Guillaume Bourque ${ }^{126,127}$, Paul C. Boutros ${ }^{47,103,128,129}$, G. Steven Bova ${ }^{130}$, David T. Bowen ${ }^{2,131}$, Reanne Bowlby ${ }^{20}$, David D. L. Bowtell ${ }^{21}$, Sandrine Boyault ${ }^{132}$, Rich Boyce ${ }^{68}$, Jeffrey Boyd ${ }^{133}$, Alvis Brazma ${ }^{68}$, Paul Brennan ${ }^{134}$, Daniel S. Brewer ${ }^{135,136}$, Arie B. Brinkman ${ }^{137}$, Robert G. Bristow ${ }^{103,138,139,140,141}$, Russell R. Broaddus ${ }^{16}$, Jane E. Brock ${ }^{142}$, Malcolm Brock ${ }^{143}$, Annegien Broeks ${ }^{144}$, Angela N. Brooks ${ }^{58,95,145,146}$, Denise Brooks ${ }^{20}$, Benedikt Brors ${ }^{147,148,149}$, Søren Brunak ${ }^{150,151}$, Timothy J. C. Bruxner ${ }^{32,152}$, Alicia L. Bruzos ${ }^{22,23,24}$, Alex Buchanan ${ }^{153}$, Ivo Buchhalter ${ }^{108,154,155}$ Christiane Buchholz ${ }^{156}$, Susan Bullman ${ }^{58,95}$, Hazel Burke ${ }^{157}$, Birgit Burkhardt ${ }^{158}$, Kathleen H. Burns ${ }^{159,160}$, John Busanovich ${ }^{58,161}$, Carlos D. Bustamante ${ }^{162,163}$, Adam P. Butler ${ }^{2}$, Atul J. Butte ${ }^{164}$, Niall J. Byrne ${ }^{29}$, Anne-Lise Børresen-Dale ${ }^{52,165}$, Samantha J. Caesar-Johnson ${ }^{166}$, Andy Cafferkey ${ }^{68}$, Declan Cahill ${ }^{167}$, Claudia Calabrese ${ }^{68,168}$, Carlos Caldas $^{169,170}$, Fabien Calvo ${ }^{171}$, Niedzica Camacho ${ }^{115}$, Peter J. Campbell ${ }^{2,172,173,721 *}$, Elias Campo ${ }^{174,175}$ Cinzia Cantù ${ }^{114}$, Shaolong Cao ${ }^{9}$, Thomas E. Carey ${ }^{176}$, Joana Carlevaro-Fita ${ }^{177,178,179}$, Rebecca Carlsen ${ }^{20}$, Ivana Cataldo ${ }^{99,14}$, Mario Cazzola ${ }^{180}$, Jonathan Cebon ${ }^{80}$, Robert Cerfolio $^{181}$, Dianne E. Chadwick ${ }^{182}$, Dimple Chakravarty ${ }^{183}$, Don Chalmers ${ }^{184}$ Calvin Wing Yiu Chan ${ }^{154,185}$, Kin Chan ${ }^{186}$, Michelle Chan-Seng-Yue ${ }^{74}$, Vishal S. Chandan ${ }^{187}$, David K. Chang ${ }^{56,104}$, Stephen J. Chanock ${ }^{188}$, Lorraine A. Chantrill ${ }^{104,189}$, Aurélien Chateigner ${ }^{29,190}$, Nilanjan Chatterjee ${ }^{75,191}$, Kazuaki Chayama ${ }^{8}$, Hsiao-Wei Chen ${ }^{33,42}$, Jieming Chen ${ }^{164}$, Ken Chen ${ }^{10}$, Yiwen Chen ${ }^{9}$, Zhaohong Chen ${ }^{192}$, Andrew D. Cherniack ${ }^{58,95}$, Jeremy Chien ${ }^{193}$, Yoke-Eng Chiew ${ }^{194,195}$, Suet-Feung Chin ${ }^{169,170}$, Juok $\mathrm{Cho}^{58}$, Sunghoon Cho ${ }^{196}$, Jung Kyoon Choi ${ }^{197}$, Wan Choi ${ }^{198}$, Christine Chomienne ${ }^{199}$, Zechen Chong ${ }^{9,200}$, Su Pin Choo ${ }^{201}$, Angela Chou ${ }^{104,194}$, Angelika N. Christ ${ }^{32}$, Elizabeth L. Christie ${ }^{21}$, Eric Chuah ${ }^{20}$, Carrie Cibulskis ${ }^{58}$, Kristian Cibulskis ${ }^{58}$, Sara Cingarlini ${ }^{202}$, Peter Clapham ${ }^{2}$, Alexander Claviez ${ }^{203}$, Sean Cleary ${ }^{74,204}$, Nicole Cloonan ${ }^{205}$, Marek Cmero 206,207,208, Colin C. Collins ${ }^{209}$, Ashton A. Connor ${ }^{204,210}$, Susanna L. Cooke ${ }^{56}$, Colin S. Cooper ${ }^{115,135,136,211}$, Leslie Cope ${ }^{75}$, Vincenzo Corbo ${ }^{62,14}$, Matthew G. Cordes ${ }^{37,212}$, Stephen M. Cordner ${ }^{213}$, Isidro Cortés-Ciriano ${ }^{214,215,216}$, Kyle Covington ${ }^{217}$, Prue A. Cowin ${ }^{218}$, Brian Craft ${ }^{146}$, David Craft ${ }^{58,219}$, Chad J. Creighton ${ }^{220}$, Yupeng Cun ${ }^{221}$, Erin Curley ${ }^{222}$, Ioana Cutcutache ${ }^{16,117}$, Karolina Czajka ${ }^{223}$, Bogdan Czerniak ${ }^{16,224}$, Rebecca A. Dagg ${ }^{25}$, Ludmila Danilova ${ }^{75}$, Maria Vittoria Davi ${ }^{226}$, Natalie R. Davidson ${ }^{27,228,229,230,231}$, Helen Davies ${ }^{2,232,233}$, Ian J. Davis ${ }^{234}$, Brandi N. Davis-Dusenbery ${ }^{235}$, Kevin J. Dawson ${ }^{2}$, Francisco M. De La Vega ${ }^{162,163,236}$, Ricardo De Paoli-Iseppi ${ }^{157}$, Timothy Defreitas ${ }^{58}$, Angelo P. Dei $\operatorname{Tos}^{237}$, Olivier Delaneau ${ }^{238,239,240}$, John A. Demchok ${ }^{166}$, Jonas Demeulemeester ${ }^{241,242}$, German M. Demidov $84,124,243$, Deniz Demircioğlu ${ }^{244,245}$, Nening M. Dennis ${ }^{167}$, Robert E. Denroche ${ }^{74}$, Stefan C. Dentro ${ }^{2,241,246}$, Nikita Desai ${ }^{247,248}$, Vikram Deshpande ${ }^{110}$, Amit G. Deshwar ${ }^{249}$, Christine Desmedt ${ }^{250,251}$ Jordi Deu-Pons ${ }^{252,253}$, Noreen Dhalla ${ }^{20}$, Neesha C. Dhani $^{254}$, Priyanka Dhingra ${ }^{255,256}$, Rajiv Dhir ${ }^{257}$, Anthony DiBiase ${ }^{258}$, Klev Diamanti ${ }^{259}$, Li Ding ${ }^{37,55,260}$, Shuai Ding ${ }^{261}$, Huy Q. Dinh ${ }^{88}$, Luc Dirix ${ }^{262}$, HarshaVardhan Doddapaneni ${ }^{217}$, Nilgun Donmez ${ }^{209,263}$, Michelle T. Dow ${ }^{192}$, Ronny Drapkin ${ }^{264}$, Oliver Drechsel ${ }^{84,124}$, Ruben M. Drews ${ }^{170}$, Serge Serge ${ }^{2}$ Tim Dudderidge ${ }^{76,167}$, Ana Dueso-Barroso ${ }^{70,265}$, Andrew J. Dunford ${ }^{58}$, Michael Dunn ${ }^{266}$, Lewis Jonathan Dursi ${ }^{47,267}$, Fraser R. Duthie ${ }^{56,268}$, Ken Dutton-Regester ${ }^{269}$, Jenna Eagles ${ }^{223}$, Douglas F. Easton ${ }^{270,271}$, Stuart Edmonds ${ }^{272}$, Paul A. Edwards ${ }^{170,273}$, Sandra E. Edwards ${ }^{115}$, Rosalind A. Eeles ${ }^{115,167}$, Anna Ehinger ${ }^{274}$, Juergen Eils ${ }^{275,276}$, Roland Eils ${ }^{154,155,275,276}$, Adel El-Naggar ${ }^{16,224}$, Matthew Eldridge ${ }^{170}$, Kyle Ellrott ${ }^{153}$, Serap Erkek ${ }^{168}$, Georgia Escaramis $^{124,277,278}$, Shadrielle M. G. Espiritu ${ }^{47}$, Xavier Estivill24,279, Dariush Etemadmoghadam ${ }^{21}$, Jorunn E. Eyfjord ${ }^{280}$, Bishoy M. Faltas ${ }^{231}$, Daiming Fan ${ }^{281}$, Yu Fan ${ }^{9}$, William C. Faquin ${ }^{110}$, Claudiu Farcas ${ }^{192}$, Matteo Fassan ${ }^{282}$, Aquila Fatima ${ }^{96}$, Francesco Favero ${ }^{283}$, Nodirjon Fayzullaev ${ }^{29}$, Ina Felau ${ }^{166}$, Sian Fereday ${ }^{21}$, Martin L. Ferguson ${ }^{284}$, Vincent Ferretti ${ }^{29,285}$, Lars Feuerbach ${ }^{147}$, Matthew A. Field ${ }^{286}$, J. Lynn Fink ${ }^{32,70}$, Gaetano Finocchiaro ${ }^{287}$, Cyril Fisher ${ }^{167}$, Matthew W. Fittall ${ }^{241}$,
Anna Fitzgerald ${ }^{288}$, Rebecca C. Fitzgerald ${ }^{233}$, Adrienne M. Flanagan ${ }^{289}$, Neil E. Fleshner $^{290}$, Paul Flicek ${ }^{68}$, John A. Foekens ${ }^{291}$, Kwun M. Fong ${ }^{292}$, Nuno A. Fonseca ${ }^{68,293}$, Christopher S. Foster ${ }^{294,295}$, Natalie S. Fox ${ }^{47}$, Michael Fraser ${ }^{47}$, Scott Frazer ${ }^{58}$, Milana Frenkel-Morgenstern ${ }^{296}$, William Friedman ${ }^{297}$, Joan Frigola ${ }^{252}$, Catrina C. Fronick ${ }^{37,212}$, Akihiro Fujimoto ${ }^{121}$, Masashi Fujita ${ }^{121}$, Masashi Fukayama ${ }^{298}$, Lucinda A. Fulton ${ }^{37}$, Robert S. Fulton ${ }^{37,55,260}$, Mayuko Furuta ${ }^{121}$, P. Andrew Futreal ${ }^{26}$, Anja Füllgrabe ${ }^{68}$, Stacey B. Gabriel ${ }^{58}$, Steven Gallinger ${ }^{74,204,210}$, Carlo Gambacorti-Passerini ${ }^{299}$, Jianjiong Gao ${ }^{42}$, Shengjie Gao $^{300}$, Levi Garraway ${ }^{95}$, Øystein Garred ${ }^{301}$, Erik Garrison ${ }^{2}$, Dale W. Garsed ${ }^{21}$, Nils Gehlenborg ${ }^{58,302}$, Josep L. L. Gelpi ${ }^{70,303}$, Joshy George ${ }^{28}$, Daniela S. Gerhard ${ }^{304}$, Clarissa Gerhauser $^{305}$, Jeffrey E. Gershenwald ${ }^{306,307}$, Mark Gerstein ${ }^{308,309,310}$, Moritz Gerstung ${ }^{68,168}$, Gad Getz ${ }^{58,97,311,312,721 *}$, Mohammed Ghori' ${ }^{2}$, Ronald Ghossein ${ }^{313}$, Nasra H. Giama ${ }^{314}$, Richard A. Gibbs ${ }^{217}$, Bob Gibson ${ }^{78}$, Anthony J. Gill ${ }^{104,315}$, Pelvender Gill ${ }^{316}$, Dilip D. Giri ${ }^{313}$, Dominik Glodzik2 ${ }^{2}$, Vincent J. Gnanapragasam ${ }^{172,317}$, Maria Elisabeth Goebler $^{318}$, Mary J. Goldman ${ }^{146}$, Carmen Gomez ${ }^{319}$, Santiago Gonzalez ${ }^{68,168}$, Abel Gonzalez-Perez ${ }^{252,253,320}$, Dmitry A. Gordenin ${ }^{321}$, James Gossage ${ }^{322}$, Kunihito Gotoh ${ }^{323}$, Ramaswamy Govindan ${ }^{55}$, Dorthe Grabau ${ }^{324}$, Janet S. Graham ${ }^{56,325}$, Robert C. Grant ${ }^{74,210}$, Anthony R. Green ${ }^{273}$, Eric Green ${ }^{326}$, Liliana Greger ${ }^{68}$, Nicola Grehan ${ }^{233}$, Sonia Grimaldi14, Sean M. Grimmond ${ }^{327}$, Robert L. Grossman ${ }^{328}$, Adam Grundhoff ${ }^{14,329}$, Gunes Gundem ${ }^{3}$, Qianyun Guo ${ }^{330}$, Manaswi Gupta ${ }^{58}$, Shailja Gupta ${ }^{331}$, Ivo G. Gut ${ }^{83,84}$, Marta Gut ${ }^{83,84}$, Jonathan Göke ${ }^{244,332}$, Gavin Ha ${ }^{58}$, Andrea Haake ${ }^{30}$, David Haan ${ }^{145}$, Siegfried Haas ${ }^{122}$, Kerstin Haase ${ }^{241}$, James E. Haber ${ }^{333}$, Nina Habermann ${ }^{168}$, Faraz Hach ${ }^{209,334}$, Syed Haider ${ }^{47}$, Natsuko Hama ${ }^{39}$, Freddie C. Hamdy ${ }^{316}$, Anne Hamilton ${ }^{218}$, Mark P. Hamilton ${ }^{335}$, Leng Han ${ }^{336}$, George B. Hanna ${ }^{337}$, Martin Hansmann ${ }^{338}$, Nicholas J. Haradhvala ${ }^{58,10}$, Olivier Harismendy ${ }^{19,339}$, Ivon Harliwong ${ }^{32}$, Arif O. Harmanci ${ }^{310,340}$, Eoghan Harrington ${ }^{341}$, Takanori Hasegawa $^{342}$, David Haussler ${ }^{146,343}$, Steve Hawkins ${ }^{170}$, Shinya Hayami ${ }^{344}$, Shuto Hayashi ${ }^{342}$, D. Neil Hayes ${ }^{57,345,346}$, Stephen J. Hayes ${ }^{347,348}$, Nicholas K. Hayward ${ }^{157,269}$, Steven Hazell ${ }^{167}$, Yao He ${ }^{349,350}$, Allison P. Heath ${ }^{351}$, Simon C. Heath ${ }^{83,84}$, David Hedley ${ }^{254}$, Apurva M. Hegde ${ }^{352}$, David I. Heiman ${ }^{58}$, Michael C. Heinold ${ }^{154,155}$, Zachary Heins ${ }^{3}$, Lawrence E. Heisler ${ }^{78}$, Eva Hellstrom-Lindberg ${ }^{353}$, Mohamed Helmy ${ }^{354}$, Seong Gu Heo ${ }^{355}$, Austin J. Hepperla ${ }^{57}$, José María Heredia-Genestar ${ }^{356}$, Carl Herrmann ${ }^{154,155,357}$, Peter Hersey ${ }^{157}$, Julian M. Hess ${ }^{58,358}$, Holmfridur Hilmarsdottir ${ }^{280}$, Jonathan Hinton ${ }^{2}$, Satoshi Hirano ${ }^{359}$, Nobuyoshi Hiraoka ${ }^{360}$, Katherine A. Hoadley ${ }^{57,361}$, Asger Hobolth ${ }^{100,330}$, Ermin Hodzic ${ }^{263}$, Jessica I. Hoell ${ }^{119}$, Steve Hoffmann ${ }^{92,93,94,362}$, Oliver Hofmann ${ }^{363}$, Andrea Holbrook ${ }^{98}$, Aliaksei Z. Holik ${ }^{124}$, Michael A. Hollingsworth ${ }^{364}$, Oliver Holmes ${ }^{152,269}$, Robert A. Holt ${ }^{20}$, Chen Hong ${ }^{147,185}$, Eun Pyo Hong ${ }^{355}$, Jongwhi H. Hong ${ }^{365}$, Gerrit K. Hooijer ${ }^{366}$, Henrik Hornshøj ${ }^{101}$, Fumie Hosoda ${ }^{39}$, Yong Hou ${ }^{300,367}$, Volker Hovestadt ${ }^{368}$, William Howat ${ }^{172}$, Alan P. Hoyle ${ }^{57}$, Ralph H. Hruban ${ }^{75}$, Jianhong Hu${ }^{217}$, Taobo $\mathrm{Hu}^{369}$, Xing Hua ${ }^{188}$, Kuan-lin Huang ${ }^{37,370}$, Mei Huang ${ }^{113}$, Mi Ni Huang ${ }^{116,17}$, Vincent Huang ${ }^{47}$, Yi Huang ${ }^{371,372}$, Wolfgang Huber ${ }^{168}$, Thomas J. Hudson ${ }^{223,373}$, Michael Hummel ${ }^{374}$, Jillian A. Hung ${ }^{194,195}$ David Huntsman ${ }^{375}$, Ted R. Hupp ${ }^{376}$, Jason Huse ${ }^{3}$, Matthew R. Huska ${ }^{377}$, Barbara Hutter ${ }^{108,148,149}$, Carolyn M. Hutter ${ }^{326}$, Daniel Hübschmann ${ }^{155,275,378,379,380,}$ Christine A. lacobuzio-Donahue ${ }^{313}$, Charles David Imbusch ${ }^{147}$, Marcin Imielinski ${ }^{381,382}$, Seiya Imoto ${ }^{342}$, William B. Isaacs ${ }^{383}$, Keren Isaev ${ }^{47,103}$, Shumpei Ishikawa ${ }^{384}$, Murat Iskar ${ }^{368}$, S. M. Ashiqul Islam ${ }^{192}$, Michael Ittmann ${ }^{385,386,387}$, Sinisa Ivkovic ${ }^{235}$, Jose M. G. Izarzugaza ${ }^{388}$, Jocelyne Jacquemier ${ }^{389}$, Valerie Jakrot ${ }^{157}$, Nigel B. Jamieson ${ }^{56,106,390}$, Gun Ho Jang ${ }^{74}$, Se Jin Jang ${ }^{391}$, Joy C. Jayaseelan ${ }^{217}$, Reyka Jayasinghe ${ }^{37}$, Stuart R. Jefferys ${ }^{57}$, Karine Jegalian $^{392}$, Jennifer L. Jennings ${ }^{393}$, Seung-Hyup Jeon ${ }^{198}$, Lara Jerman ${ }^{168,394}$, Yuan Ji ${ }^{395,396}$, Wei Jiao ${ }^{47}$, Peter A. Johansson ${ }^{269}$, Amber L. Johns ${ }^{104}$, Jeremy Johns ${ }^{223}$, Rory Johnson ${ }^{178,397}$, Todd A. Johnson ${ }^{120}$, Clemency Jolly ${ }^{241}$, Yann Joly ${ }^{398}$, Jon G. Jonasson ${ }^{280}$, Corbin D. Jones ${ }^{399}$, David R. Jones ${ }^{2}$, David T. W. Jones ${ }^{400,401}$, Nic Jones ${ }^{402}$, Steven J. M. Jones ${ }^{20}$, Jos Jonkers ${ }^{144}$, Young Seok Ju ${ }^{2,197}$, Hartmut Juhl ${ }^{403}$, Jongsun Jung ${ }^{404}$, Malene Juul ${ }^{101}$, Randi Istrup Juul ${ }^{101}$, Sissel Juul ${ }^{341}$, Natalie Jäger ${ }^{154}$, Rolf Kabbe ${ }^{154}$, Andre Kahles ${ }^{227,228,229,230,405}$, Abdullah Kahraman ${ }^{406,407,408}$, Vera B. Kaiser ${ }^{409}$, Hojabr Kakavand ${ }^{157}$, Sangeetha Kalimuthu ${ }^{74}$, Christof von Kalle ${ }^{379}$, Koo Jeong Kang ${ }^{6}$, Katalin Karaszi ${ }^{316}$, Beth Karlan ${ }^{410}$, Rosa Karlić ${ }^{411}$, Dennis Karsch ${ }^{412}$, Katayoon Kasaian ${ }^{20}$, Karin S. Kassahn ${ }^{32,413}$, Hitoshi Katai ${ }^{414}$, Mamoru Kato ${ }^{415}$, Hiroto Katoh ${ }^{384}$, Yoshiiku Kawakami ${ }^{8}$, Jonathan D. Kay ${ }^{38}$, Stephen H. Kazakoff ${ }^{152,269}$, Marat D. Kazanov ${ }^{416,417,418}$, Maria Keays ${ }^{68}$, Electron Kebebew ${ }^{419,420}$, Richard F. Kefford ${ }^{421}$, Manolis Kellis ${ }^{58,422}$, James G. Kench ${ }^{104,315,423}$, Catherine J. Kennedy ${ }^{194,195}$, Jules N. A. Kerssemakers ${ }^{154}$, David Khoo ${ }^{224}$, Vincent Khoo ${ }^{167}$ Narong Khuntikeo ${ }^{34,424}$, Ekta Khurana ${ }^{255,256,425,426}$, Helena Kilpinen ${ }^{38}$, Hark Kyun Kim ${ }^{427}$, Hyung-Lae Kim ${ }^{428}$, Hyung-Yong Kim ${ }^{389}$, Hyunghwan Kim ${ }^{198}$, Jaegil Kim ${ }^{58}$, Jihoon Kim ${ }^{429}$, Jong K. Kim ${ }^{430}$, Youngwook Kim ${ }^{431,432}$, Tari A. King ${ }^{433,434,435}$, Wolfram Klapper ${ }^{50}$, Kortine Kleinheinz ${ }^{154,155}$, Leszek J. Klimczak ${ }^{436}$, Stian Knappskog ${ }^{2,437}$, Michael Knebaa', Bartha M. Knoppers ${ }^{398}$, Youngil Koh ${ }^{438,439}$, Jan Komorowski ${ }^{259,440}$, Daisuke Komura ${ }^{384}$, Mitsuhiro Komura ${ }^{342}$, Gu Kong ${ }^{389}$, Marcel Kool ${ }^{400,441}$, Jan O. Korbel ${ }^{68,168,721 *}$, Viktoriya Korchina ${ }^{217}$, Andrey Korshunov ${ }^{441}$, Michael Koscher ${ }^{441}$, Roelof Koster ${ }^{442}$, Zsofia Kote-Jarai ${ }^{115}$, Antonios Koures ${ }^{192}$, Milena Kovacevic ${ }^{235}$, Barbara Kremeyer ${ }^{2}$, Helene Kretzmer ${ }^{93,94}$, Markus Kreuz ${ }^{443}$, Savitri Krishnamurthy ${ }^{16,444}$, Dieter Kube ${ }^{445}$, Kiran Kumar ${ }^{58}$, Pardeep Kumar ${ }^{167}$, Sushant Kumar ${ }^{309,310}$, Yogesh Kumar ${ }^{369}$, Ritika Kundra ${ }^{33,42}$, Kirsten Kübler ${ }^{58,97,10}$, Ralf Küppers ${ }^{446}$, Jesper Lagergren ${ }^{353,447}$, Phillip H. Lai ${ }^{98}$, Peter W. Laird ${ }^{448}$, Sunil R. Lakhani ${ }^{449}$, Christopher M. Lalansingh ${ }^{47}$, Emilie Lalonde ${ }^{47}$, Fabien C. Lamaze ${ }^{47}$, Adam Lambert ${ }^{316}$, Eric Lander ${ }^{58}$, Pablo Landgraf ${ }^{450,451}$, Luca Landoni ${ }^{34}$, Anita Langerød ${ }^{52}$, Andrés Lanzós ${ }^{18,179,397}$, Denis Larsimont ${ }^{452}$, Erik Larsson ${ }^{453}$, Mark Lathrop ${ }^{127}$, Loretta M. S. Lau ${ }^{454}$, Chris Lawerenz ${ }^{276}$, Rita T. Lawlor ${ }^{14}$, Michael S. Lawrence ${ }^{58,110,120}$, Alexander J. Lazar ${ }^{16,26}$, Ana Mijalkovic Lazic ${ }^{235}$, Xuan Le ${ }^{455}$, Darlene $\mathrm{Lee}^{20}$, Donghoon Lee ${ }^{310}$, Eunjung Alice Lee ${ }^{456}$, Hee Jin Lee ${ }^{391}$ Jake June-Koo Lee ${ }^{214,216}$, Jeong-Yeon Lee ${ }^{457}$, Juhee Lee ${ }^{458}$, Ming Ta Michael Lee ${ }^{459}$, 
Henry Lee-Six ${ }^{2}$, Kjong-Van Lehmann ${ }^{227,228,229,230,405}$, Hans Lehrach ${ }^{460}$, Dido Lenze $^{374}$ Conrad R. Leonard ${ }^{152,269}$, Daniel A. Leongamornlert ${ }^{2,115}$, Ignaty Leshchiner ${ }^{58}$, Louis Letourneau ${ }^{461}$, Ivica Letunic ${ }^{462}$, Douglas A. Levine ${ }^{3,463}$, Lora Lewis ${ }^{217}$, Tim Ley ${ }^{464}$ Chang $\mathrm{Li}^{300,367}$, Constance H. $\mathrm{Li}^{47,103}$, Haiyan Irene $\mathrm{Li}^{20}$, Jun $\mathrm{Li}^{9}$, Lin $\mathrm{Li}^{300}$, Shantao $\mathrm{Li}^{310}$, Siliang $\mathrm{Li}^{300,367}$, Xiaobo $\mathrm{Li}^{300,367}$, Xiaotong $\mathrm{Li}^{310}$, Xinyue $\mathrm{Li}^{300}$, Yilong $\mathrm{Li}^{2}$, Han Liang ${ }^{9,27,465}$, Sheng-Ben Liang ${ }^{182}$, Peter Lichter ${ }^{148,368}$, Pei Lin ${ }^{58}$, Ziao Lin ${ }^{58,466}$, W. M. Linehan ${ }^{467}$, Ole Christian Lingjærde ${ }^{468}$, Dongbing Liu ${ }^{300,367}$, Eric Minwei Liu ${ }^{3,255,256}$, Fei-Fei Fei Liu ${ }^{141,469}$, Fenglin Liu ${ }^{470,471}$, Jia Liu ${ }^{472}$, Xingmin Liu ${ }^{300,367}$, Julie Livingstone ${ }^{47}$, Dimitri Livitz ${ }^{58}$, Naomi Livni ${ }^{167}$, Lucas Lochovsky ${ }^{28,309,310}$, Markus Loeffler ${ }^{443}$, Georgina V. Long ${ }^{157}$, Armando Lopez-Guillermo ${ }^{473}$, Shaoke Lou ${ }^{309,310}$, David N. Louis ${ }^{110}$, Laurence B. Lovat ${ }^{38}$, Yiling Lu ${ }^{352}$, Yong-Jie Lu ${ }^{91,474}$, Youyong Lu ${ }^{475,476,477}$, Claudio Luchini ${ }^{99}$, Ilinca Lungu ${ }^{69,74}$, Xuemei Luo ${ }^{78}$, Hayley J. Luxton ${ }^{38}$, Andy G. Lynch ${ }^{170,273,478}$, Lisa Lype ${ }^{479}$, Cristina López ${ }^{30,31}$, Carlos López-Otín ${ }^{480}$, Eric Z. Ma ${ }^{369}$, Yussanne Ma ${ }^{20}$, Gaetan MacGrogan ${ }^{481}$, Shona MacRae ${ }^{482}$, Geoff Macintyre $^{170}$, Tobias Madsen ${ }^{101}$, Kazuhiro Maejima ${ }^{121}$, Andrea Mafficini ${ }^{114}$, Dennis T. Maglinte ${ }^{98,483}$, Arindam Maitra ${ }^{111}$, Partha P. Majumder ${ }^{111}$, Luca Malcovati ${ }^{180}$, Salem Malikic ${ }^{209,263}$, Giuseppe Malleo ${ }^{34}$, Graham J. Mann ${ }^{157,94,484}$, Luisa Mantovani-Löffler ${ }^{485}$, Kathleen Marchal ${ }^{486,487}$, Giovanni Marchegiani ${ }^{34}$, Elaine R. Mardis ${ }^{37,133,488}$, Adam A. Margolin ${ }^{489}$, Maximillian G. Marin ${ }^{145}$, Florian Markowetz ${ }^{170,273}$, Julia Markowski ${ }^{377}$ Jeffrey Marks ${ }^{490}$, Tomas Marques-Bonet ${ }^{83,356,491,492}$, Marco A. Marra ${ }^{20}$, Luke Marsden ${ }^{316}$, John W. M. Martens ${ }^{291}$, Sancha Martin ${ }^{2,493}$, Jose I. Martin-Subero ${ }^{491,494}$, Iñigo Martincorena ${ }^{2}$, Alexander Martinez-Fundichely ${ }^{255,256,426}$, Yosef E. Maruvka ${ }^{58,110,358}$, R. Jay Mashl ${ }^{37,495}$, Charlie E. Massie ${ }^{170}$, Thomas J. Matthew ${ }^{145}$, Lucy Matthews ${ }^{115}$, Erik Mayer ${ }^{167,496}$, Simon Mayes ${ }^{497}$, Michael Mayo ${ }^{20}$, Faridah Mbabaali ${ }^{223}$, Karen McCune ${ }^{498}$, Ultan McDermott ${ }^{2}$, Patrick D. McGillivray ${ }^{309}$, Michael D. McLellan ${ }^{37,55,260}$, John D. McPherson ${ }^{74,223,499}$, John R. McPherson ${ }^{16,117}$, Treasa A. McPherson ${ }^{210}$, Samuel R. Meier ${ }^{58}$, Alice Meng ${ }^{500}$, Shaowu Meng ${ }^{57}$, Andrew Menzies ${ }^{2}$, Neil D. Merrett ${ }^{34,501}$, Sue Merson ${ }^{115}$, Matthew Meyerson ${ }^{58,95,97}$, William Meyerson ${ }^{310,502}$, Piotr A. Mieczkowski ${ }^{503}$, George L. Mihaiescu ${ }^{29}$, Sanja Mijalkovic ${ }^{235}$, Tom Mikkelsen ${ }^{504}$, Michele Milella ${ }^{202}$, Linda Mileshkin ${ }^{21}$, Christopher A. Miller ${ }^{37}$, David K. Miller ${ }^{32,104}$, Jessica K. Miller ${ }^{223}$, Gordon B. Mills ${ }^{505}$, Ana Milovanovic ${ }^{70}$, Sarah Minner ${ }^{506}$, Marco Miotto ${ }^{34}$, Gisela Mir Arnau ${ }^{218}$, Lisa Mirabello ${ }^{188}$, Chris Mitchell ${ }^{21}$, Thomas J. Mitchell ${ }^{2,172,273}$, Satoru Miyano ${ }^{342}$, Naoki Miyoshi ${ }^{342}$, Shinichi Mizuno ${ }^{507}$, Fruzsina Molnár-Gábor ${ }^{508}$, Malcolm J. Moore ${ }^{254}$ Richard A. Moore ${ }^{20}$, Sandro Morganella ${ }^{2}$, Quaid D. Morris ${ }^{49,469}$, Carl Morrison ${ }^{509,510}$, Lisle E. Mose ${ }^{57}$, Catherine D. Moser ${ }^{314}$, Ferran Muiños ${ }^{252,253}$, Loris Mularoni ${ }^{252,253}$, Andrew J. Mungall ${ }^{20}$, Karen Mungall ${ }^{20}$, Elizabeth A. Musgrove ${ }^{56}$, Ville Mustonen ${ }^{511,512,513}$, David Mutch $^{514}$, Francesc Muyas ${ }^{84,124,243}$, Donna M. Muzny ${ }^{217}$, Alfonso Muñoz ${ }^{68}$, Jerome Myers ${ }^{515}$, Ola Myklebost ${ }^{437}$, Peter Möller ${ }^{516}$, Genta Nagae ${ }^{4}$, Adnan M. Nagrial ${ }^{104}$, Hardeep K. Nahal-Bose ${ }^{29}$, Hitoshi Nakagama ${ }^{517}$, Hidewaki Nakagawa ${ }^{121}$, Hiromi Nakamura ${ }^{39}$, Toru Nakamura ${ }^{359}$, Kaoru Nakano ${ }^{121}$, Tannistha Nandi ${ }^{518}$, Jyoti Nangalia ${ }^{2}$, Mia Nastic ${ }^{235}$, Arcadi Navarro $^{83,356,491}$, Fabio C. P. Navarro ${ }^{308,309,519}$, David E. Neal ${ }^{170,172}$, Gerd Nettekoven ${ }^{520}$, Felicity Newell ${ }^{152,269}$, Steven J. Newhouse ${ }^{68}$, Yulia Newton ${ }^{145}$, Alvin Wei Tian $\mathbf{N g}^{521}$, Anthony $\mathrm{Ng}^{522}$, Jonathan Nicholson ${ }^{2}$, David Nicol ${ }^{167}$, Yongzhan $\mathrm{Ni}^{281,523}$, G. Petur Nielsen ${ }^{110}$, Morten Muhlig Nielsen ${ }^{101}$, Serena Nik-Zainal ${ }^{2,232,233,524}$, Michael S. Noble $^{58}$, Katia Nones $^{152,269}$, Paul A. Northcott ${ }^{525}$, Faiyaz Notta ${ }^{74,526}$, Brian D. O'Connor ${ }^{29,527}$, Peter O'Donnell ${ }^{528}$, Maria O'Donovan ${ }^{233}$, Sarah O'Meara ${ }^{2}$, Brian Patrick O'Neill ${ }^{529}$, J. Robert O'Neill ${ }^{530}$, David Ocana ${ }^{68}$, Angelica Ochoa ${ }^{3}$, Layla Oesper ${ }^{531}$, Christopher Ogden ${ }^{167}$, Hideki Ohdan ${ }^{8}$, Kazuhiro Ohi ${ }^{342}$, Lucila Ohno-Machado ${ }^{192}$, Karin A. Oien ${ }^{509,532}$, Akinyemi I. Ojesina ${ }^{533,534,535}$ Hidenori Ojima ${ }^{536}$, Takuji Okusaka ${ }^{537}$, Larsson Omberg ${ }^{538}$, Choon Kiat Ong ${ }^{539}$, Stephan Ossowski ${ }^{84,124,243}$, German Ott ${ }^{540}$, B. F. Francis Ouellette ${ }^{29,541}$, Christine P'ng ${ }^{47}$, Marta Paczkowska ${ }^{47}$, Salvatore Paiella ${ }^{34}$, Chawalit Pairojkul ${ }^{509}$, Marina Pajic ${ }^{104}$, Qiang Pan-Hammarström ${ }^{300,542}$, Elli Papaemmanuil' ${ }^{2}$, Irene Papatheodorou ${ }^{68}$, Nagarajan Paramasivam ${ }^{108,154}$, Ji Wan Park ${ }^{355}$, Joong-Won Park ${ }^{543}$, Keunchil Park ${ }^{544,545}$, Kiejung Park ${ }^{546}$, Peter J. Park ${ }^{214,216}$, Joel S. Parker ${ }^{503}$, Simon L. Parsons ${ }^{45}$, Harvey Pass ${ }^{547}$, Danielle Pasternack ${ }^{223}$, Alessandro Pastore ${ }^{227}$, Ann-Marie Patch ${ }^{152,269}$, Iris Pauporté ${ }^{199}$, Antonio Pea ${ }^{34}$, John V. Pearson ${ }^{152,269}$, Chandra Sekhar Pedamallu ${ }^{58,95,97}$, Jakob Skou Pedersen ${ }^{101,330}$, Paolo Pederzoli ${ }^{34}$, Martin Peifer ${ }^{221}$, Nathan A. Pennell ${ }^{548}$, Charles M. Perou ${ }^{51,503}$, Marc D. Perry ${ }^{29,549}$, Gloria M. Petersen ${ }^{550}$, Myron Peto ${ }^{35}$, Nicholas Petrelli ${ }^{551}$, Robert Petryszak ${ }^{68}$, Stefan M. Pfister ${ }^{400,441,552}$, Mark Phillips ${ }^{398}$, Oriol Pich ${ }^{252,253}$, Hilda A. Pickett ${ }^{454}$, Todd D. Pihl ${ }^{553}$, Nischalan Pillay ${ }^{554}$, Sarah Pinder ${ }^{555}$, Mark Pinese $^{104}$, Andreia V. Pinho $^{556}$, Esa Pitkänen ${ }^{168}$, Xavier Pivot ${ }^{557}$, Elena Piñeiro-Yáñé ${ }^{12}$, Laura Planko $^{520}$, Christoph Plass ${ }^{305}$, Paz Polak ${ }^{58,97,311}$, Tirso Pons ${ }^{558}$, Irinel Popescu ${ }^{559}$, Olga Potapova ${ }^{560}$, Aparna Prasad ${ }^{84}$, Shaun R. Preston ${ }^{561}$, Manuel Prinz ${ }^{154}$, Antonia L. Pritchard ${ }^{269}$, Stephenie D. Prokopec ${ }^{47}$, Elena Provenzano ${ }^{562}$, Xose S. Puente ${ }^{480}$ Sonia Puig ${ }^{113}$, Montserrat Puiggròs ${ }^{70}$, Sergio Pulido-Tamayo ${ }^{486,487}$, Gulietta M. Pupo ${ }^{194}$, Colin A. Purdie ${ }^{563}$, Michael C. Quinn ${ }^{152,269}$, Raquel Rabionet ${ }^{84,24,564}$, Janet S. Rader ${ }^{565}$, Bernhard Radlwimmer ${ }^{368}$, Petar Radovic ${ }^{235}$, Benjamin Raeder ${ }^{168}$, Keiran M. Raine ${ }^{2}$, Manasa Ramakrishna ${ }^{2}$, Kamna Ramakrishnan ${ }^{2}$, Suresh Ramalingam ${ }^{566}$, Benjamin J. Raphael ${ }^{567}$, W. Kimryn Rathmell ${ }^{568}$, Tobias Rausch $^{168}$, Guido Reifenberger ${ }^{451}$, Jüri Reimand ${ }^{47,103}$, Jorge Reis-Filho ${ }^{313}$, Victor Reuter ${ }^{313}$, Iker Reyes-Salazar ${ }^{252}$, Matthew A. Reyna ${ }^{567}$, Sheila M. Reynolds ${ }^{479}$, Esther Rheinbay ${ }^{58,97,10}$, Yasser Riazalhosseini ${ }^{127}$ Andrea L. Richardson ${ }^{96}$, Julia Richter ${ }^{30,50}$, Matthew Ringel ${ }^{569}$, Markus Ringnér ${ }^{118}$, Yasushi Rino ${ }^{570}$, Karsten Rippe ${ }^{571}$, Jeffrey Roach ${ }^{572}$, Lewis R. Roberts ${ }^{314}$, Nicola D. Roberts ${ }^{2}$, Steven A. Roberts ${ }^{573}$, A. Gordon Robertson ${ }^{20}$, Alan J. Robertson ${ }^{32}$, Javier Bartolomé Rodriguez ${ }^{70}$, Bernardo Rodriguez-Martin ${ }^{22,23,24}$, F. Germán Rodríguez-González ${ }^{291,574}$, Michael H. A. Roehrl ${ }^{44,74,103,182,575,576}$, Marius Rohde ${ }^{577}$, Hirofumi Rokutan ${ }^{415}$, Gilles Romieu ${ }^{578}$, Ilse Rooman ${ }^{104}$, Tom Roques ${ }^{212}$, Daniel Rosebrock ${ }^{58}$, Mara Rosenberg ${ }^{58,110}$, Philip C. Rosenstiel ${ }^{579}$, Andreas Rosenwald ${ }^{580}$, Edward W. Rowe ${ }^{167,581}$,
Romina Royo ${ }^{70}$, Steven G. Rozen ${ }^{11,117,582}$, Yulia Rubanova ${ }^{49,583}$, Mark A. Rubin ${ }^{397,584,585,586,587}$, Carlota Rubio-Perez ${ }^{252,253,588}$, Vasilisa A. Rudneva ${ }^{168}$, Borislav C. Rusev ${ }^{114}$, Andrea Ruzzenente ${ }^{589}$, Gunnar Rätsch ${ }^{227,228,229,230,231,405}$, Radhakrishnan Sabarinathan ${ }^{252,253,590}$, Veronica Y. Sabelnykova ${ }^{47}$, Sara Sadeghi ${ }^{20}$, S. Cenk Sahinalp ${ }^{209,263,591}$, Natalie Saini ${ }^{321}$, Mihoko Saito-Adachi ${ }^{415}$, Gordon Saksena ${ }^{58}$, Adriana Salcedo ${ }^{47}$, Roberto Salgado ${ }^{592}$, Leonidas Salichos ${ }^{309,310}$, Richard Sallari ${ }^{58}$, Charles Saller ${ }^{593}$, Roberto Salvia ${ }^{34}$, Michelle Sam ${ }^{223}$, Jaswinder S. Samra ${ }^{34,594}$, Francisco Sanchez-Vega ${ }^{33,42}$, Chris Sander ${ }^{227,595,596}$, Grant Sanders ${ }^{57}$, Rajiv Sarin ${ }^{597}$, Iman Sarrafi ${ }^{209,263}$, Aya Sasaki-Oku ${ }^{121}$, Torill Sauer $^{468}$, Guido Sauter ${ }^{506}$, Robyn P. M. Saw ${ }^{157}$, Maria Scardoni ${ }^{99}$, Christopher J. Scarlett ${ }^{104,598}$, Aldo Scarpa ${ }^{114}$, Ghislaine Scelo ${ }^{134}$, Dirk Schadendorf ${ }^{188,599}$, Jacqueline E. Schein ${ }^{20}$, Markus B. Schilhabel ${ }^{579}$, Matthias Schlesner ${ }^{154,600}$, Thorsten Schlomm ${ }^{601,602}$, Heather K. Schmidt ${ }^{37}$, Sarah-Jane Schramm ${ }^{194}$, Stefan Schreiber ${ }^{603}$ Nikolaus Schultz ${ }^{42}$, Steven E. Schumacher ${ }^{58,95,96}$, Roland F. Schwarz ${ }^{68,377,379,604}$, Richard A. Scolyer ${ }^{157,423,594}$, David Scott ${ }^{402}$, Ralph Scully ${ }^{605}$, Raja Seethala ${ }^{606}$, Ayellet V. Segre ${ }^{58,607}$, Iris Selander ${ }^{210}$, Colin A. Semple ${ }^{409}$, Yasin Senbabaoglu ${ }^{227}$, Subhajit Sengupta ${ }^{608}$, Elisabetta Sereni ${ }^{34}$, Stefano Serra ${ }^{575}$, Dennis C. Sgroi ${ }^{110}$, Mark Shackleton ${ }^{21}$, Nimish C. Shah ${ }^{172}$, Sagedeh Shahabi ${ }^{182}$, Catherine A. Shang ${ }^{288}$, Ping Shang ${ }^{157}$, Ofer Shapira ${ }^{58,96}$, Troy Shelton ${ }^{222}$, Ciyue Shen ${ }^{595,596}$, Hui Shen ${ }^{609}$, Rebecca Shepherd ${ }^{2}$, Ruian Shi ${ }^{469}$, Yan Shi ${ }^{57}$, Yu-Jia Shiah ${ }^{47}$, Tatsuhiro Shibata ${ }^{39,610}$, Juliann Shih $^{58,95}$, Eigo Shimizu ${ }^{342}$, Kiyo Shimizu ${ }^{611}$, Seung Jun Shin ${ }^{612}$, Yuichi Shiraishi ${ }^{342}$, Tal Shmaya ${ }^{236}$, Ilya Shmulevich ${ }^{479}$, Solomon I. Shorser ${ }^{47}$, Charles Short ${ }^{68}$, Raunak Shrestha ${ }^{209}$, Suyash S. Shringarpure ${ }^{163}$, Craig Shriver $^{613}$, Shimin Shuai ${ }^{47,48}$, Nikos Sidiropoulos ${ }^{574}$, Reiner Siebert ${ }^{31,614}$, Anieta M. Sieuwerts ${ }^{291}$, Lina Sieverling ${ }^{147,185}$, Sabina Signoretti ${ }^{142,615}$, Katarzyna O. Sikora ${ }^{114}$, Michele Simbolo ${ }^{62}$, Ronald Simon ${ }^{506}$, Janae V. Simons ${ }^{57}$, Jared T. Simpson ${ }^{47,583}$, Peter T. Simpson ${ }^{449}$, Samuel Singer ${ }^{34,434}$, Nasa Sinnott-Armstrong ${ }^{58,163}$, Payal Sipahimalani ${ }^{20}$, Tara J. Skelly ${ }^{361}$, Marcel Smid ${ }^{291}$, Jaclyn Smith ${ }^{616}$, Karen Smith-McCune ${ }^{498}$, Nicholas D. Socci ${ }^{227}$, Heidi J. Sofia ${ }^{326}$, Matthew G. Soloway ${ }^{57}$, Lei Song ${ }^{188}$, Anil K. Sood ${ }^{617,618,619}$, Sharmila Sothi ${ }^{620}$, Christos Sotiriou ${ }^{192}$, Cameron M. Soulette ${ }^{145}$, Paul N. Span ${ }^{621}$, Paul T. Spellman ${ }^{35}$, Nicola Sperandio ${ }^{114}$, Andrew J. Spillane ${ }^{157}$, Oliver Spiro ${ }^{58}$, Jonathan Spring ${ }^{622}$, Johan Staaf ${ }^{118}$, Peter F. Stadler ${ }^{92,93,94}$, Peter Staib ${ }^{623}$, Stefan G. Stark ${ }^{228,230,612,624}$, Lucy Stebbings ${ }^{2}$, Ólafur Andri Stefánsson ${ }^{625}$, Oliver Stegle ${ }^{68,168,626}$, Lincoln D. Stein ${ }^{47,48,721 *}$, Alasdair Stenhouse ${ }^{627}$, Chip Stewart ${ }^{58}$, Stephan Stilgenbauer ${ }^{628}$, Miranda D. Stobbe ${ }^{83,84}$, Michael R. Stratton ${ }^{2}$, Jonathan R. Stretch ${ }^{157}$, Adam J. Struck ${ }^{489}$, Joshua M. Stuart ${ }^{145,146,721 *}$, Henk G. Stunnenberg ${ }^{367,629}$, Hong Su ${ }^{300,367}$, Xiaoping Su ${ }^{16}$, Ren X. Sun ${ }^{47}$, Stephanie Sungalee ${ }^{168}$, Hana Susak ${ }^{84,124}$, Akihiro Suzuki ${ }^{4,630}$, Fred Sweep ${ }^{631}$, Monika Szczepanowski ${ }^{50}$, Holger Sültmann ${ }^{148,149,632}$, Takashi Yugawa ${ }^{611}$, Angela $\operatorname{Tam}^{20}$, David Tamborero ${ }^{252,253}$, Benita Kiat Tee $\operatorname{Tan}^{633}$, Donghui $\operatorname{Tan}^{503}$, Patrick Tan ${ }^{117,518,582,634}$, Hiroko Tanaka ${ }^{342}$, Hirokazu Taniguchi ${ }^{60}$, Tomas J. Tanskanen ${ }^{635}$, Maxime Tarabichi ${ }^{2,241}$, Roy Tarnuzzer ${ }^{166}$, Patrick Tarpey ${ }^{636}$, Morgan L. Taschuk ${ }^{78}$, Kenji Tatsuno $^{4}$, Simon Tavaré ${ }^{170,637}$, Darrin F. Taylor ${ }^{32}$, Amaro Taylor-Weiner ${ }^{58}$, Jon W. Teague ${ }^{2}$, Bin Tean Teh ${ }^{17,582,634,638,639}$, Varsha Tembe ${ }^{194}$, Javier Temes ${ }^{22,23}$, Kevin Thai ${ }^{29}$, Sarah P. Thayer ${ }^{364}$, Nina Thiessen ${ }^{20}$, Gilles Thomas ${ }^{640}$, Sarah Thomas ${ }^{167}$, Alan Thompson ${ }^{167}$, Alastair M. Thompson ${ }^{627}$, John F. F. Thompson ${ }^{157}$, R. Houston Thompson ${ }^{641}$, Heather Thorne ${ }^{21}$, Leigh B. Thorne ${ }^{113}$, Adrian Thorogood ${ }^{398}$, Grace Tiao ${ }^{58}$, Nebojsa Tijanic ${ }^{235}$, Lee E. Timms ${ }^{223}$, Roberto Tirabosco ${ }^{642}$, Marta Tojo ${ }^{24,643}$, Stefania Tommasi ${ }^{644}$, Christopher W. Toon ${ }^{104}$, Umut H. Toprak ${ }^{15,645}$, David Torrents ${ }^{70,491}$, Giampaolo Tortora ${ }^{646,647}$, Jörg Tost ${ }^{648}$, Yasushi Totoki ${ }^{39}$, David Townend ${ }^{649}$, Nadia Traficante ${ }^{21}$, Isabelle Treilleux ${ }^{650,651}$,' Jean-Rémi Trotta ${ }^{83}$, Lorenz H. P. Trümper ${ }^{445}$, Ming Tsao ${ }^{45,526}$, Tatsuhiko Tsunoda ${ }^{120,652,653,654}$, Jose M. C. Tubio ${ }^{22,23,24,655}$, Olga Tucker ${ }^{656}$, Richard Turkington ${ }^{657}$, Daniel J. Turner ${ }^{497}$, Andrew Tutt ${ }^{96}$, Masaki Ueno ${ }^{344}$, Naoto T. Ueno ${ }^{658}$, Christopher Umbricht ${ }^{77,159,659}$, Husen M. Umer ${ }^{259,660}$, Timothy J. Underwood ${ }^{661}$, Lara Urban ${ }^{68,168}$, Tomoko Urushidate ${ }^{610}$, Tetsuo Ushiku ${ }^{298}$, Liis Uusküla-Reimand ${ }^{662,663}$, Alfonso Valencia ${ }^{70,491}$, David J. Van Den Berg ${ }^{98}$, Steven Van Laere ${ }^{262}$, Peter Van Loo ${ }^{241,242}$, Erwin G. Van Meir ${ }^{664}$, Gert G. Van den Eynden ${ }^{262}$, Theodorus Van der Kwast ${ }^{44}$, Naveen Vasudev ${ }^{61}$, Miguel Vazquez ${ }^{70,665}$, Ravikiran Vedururu ${ }^{218}$, Umadevi Veluvolu ${ }^{503}$, Shankar Vembu ${ }^{469,666}$, Lieven P. C. Verbeke ${ }^{487,667}$, Peter Vermeulen ${ }^{262}$, Clare Verrill $^{316,668}$, Alain Viari ${ }^{144}$, David Vicente ${ }^{70}$, Caterina Vicentini ${ }^{114},{ }^{14}$. VijayRaghavan ${ }^{331}$, Juris Viksna ${ }^{669}$, Ricardo E. Vilain ${ }^{670}$, Izar Villasante ${ }^{70}$, Anne Vincent-Salomon ${ }^{629}$, Tapio Visakorpi ${ }^{130}$, Douglas Voet ${ }^{58}$, Paresh Vyas ${ }^{269,316}$, Ignacio Vázquez-García ${ }^{2,671,672,673}$, Nick M. Waddell ${ }^{152}$, Nicola Waddell ${ }^{152,269}$, Claes Wadelius ${ }^{674}$, Lina Wadi ${ }^{47}$, Rabea Wagener ${ }^{30,31}$, Jeremiah A. Wala ${ }^{58,95,96,97}$, Jian Wang ${ }^{300}$, Jiayin Wang ${ }^{37,372,675}$, Linghua Wang ${ }^{217}$, Qi Wang ${ }^{441}$, Wenyi Wang ${ }^{9}$, Yumeng Wang ${ }^{9,27}$, Zhining Wang ${ }^{166}$, Paul M. Waring ${ }^{509}$ Hans-Jörg Warnatz ${ }^{460}$, Jonathan Warrell ${ }^{309,310}$, Anne Y. Warren ${ }^{172,676}$, Sebastian M. Waszak ${ }^{168}$, David C. Wedge $^{2,246,677}$, Dieter Weichenhan ${ }^{305}$, Paul Weinberger ${ }^{678}$, John N. Weinstein ${ }^{352}$, Joachim Weischenfeldt ${ }^{168,574,601}$, Daniel J. Weisenberger ${ }^{98}$, lan Welch ${ }^{679}$, Michael C. Wendl ${ }^{37,260,680}$, Johannes Werner ${ }^{154,681}$, Justin P. Whalley ${ }^{83,682}$, David A. Wheeler ${ }^{217,683}$, Hayley C. Whitaker ${ }^{38}$, Dennis Wigle ${ }^{684}$, Matthew D. Wilkerson ${ }^{503}$, Ashley Williams $^{192}$, James S. Wilmott ${ }^{157}$, Gavin W. Wilson ${ }^{47,74}$, Julie M. Wilson ${ }^{74}$, Richard K. Wilson ${ }^{37,685}$, Boris Winterhoff ${ }^{686}$, Jeffrey A. Wintersinger ${ }^{49,354,583}$, Maciej Wiznerowicz ${ }^{687,688}$, Stephan Wolf ${ }^{689}$, Bernice H. Wong ${ }^{690}$, Tina Wong ${ }^{20,37}$, Winghing Wong ${ }^{691}$, Youngchoon Woo ${ }^{198}$, Scott Wood ${ }^{152,269}$, Bradly G. Wouters ${ }^{103}$, Adam J. Wright ${ }^{47}$, Derek W. Wright ${ }^{56,692}$, Mark H. Wright ${ }^{163}$, Chin-Lee Wu ${ }^{10}$, Dai-Ying Wu ${ }^{236}$, Guanming $\mathrm{Wu}^{693}$, Jianmin $\mathrm{Wu}^{104}$, Kui Wu ${ }^{300,367}$, Yang Wu ${ }^{116,117}$, Zhenggang $\mathrm{Wu}^{369}$, Liu Xi ${ }^{217}$, Tian Xia ${ }^{694}$, Qian Xiang ${ }^{29}$, Xiao Xiao ${ }^{372}$, Rui Xing ${ }^{477}$, Heng Xiong ${ }^{300,367}$, Qinying Xu ${ }^{152,269}$, Yanxun $X u^{695}$, Hong Xue ${ }^{369}$, Shinichi Yachida ${ }^{39,696}$, Sergei Yakneen ${ }^{168,697}$, Rui Yamaguchi ${ }^{342}$, Takafumi N. Yamaguchi ${ }^{47}$, Masakazu Yamamoto ${ }^{41}$, Shogo Yamamoto ${ }^{4}$, Hiroki Yamaue ${ }^{344}$, Fan Yang ${ }^{469}$, Huanming Yang ${ }^{300}$, Jean Y. Yang ${ }^{698}$, Liming Yang ${ }^{166}$, Lixing Yang ${ }^{699,700}$, Shanlin Yang ${ }^{261}$, Tsun-Po Yang ${ }^{221}$, Yang Yang ${ }^{336}$, Xiaotong Yao ${ }^{382,701}$, Marie-Laure Yaspo ${ }^{460}$, 
Lucy Yates ${ }^{2}$, Christina Yau ${ }^{85}$, Chen Ye ${ }^{300,367}$, Kai Ye ${ }^{675,702}$, Venkata D. Yellapantula ${ }^{260,672}$

Christopher J. Yoon ${ }^{197}$, Sung-Soo Yoon ${ }^{439}$, Fouad Yousif ${ }^{47}$, Jun $\mathrm{Yu}^{703}$, Kaixian $\mathrm{Yu}^{704}$,

Willie $\mathrm{Yu}^{705}$, Yingyan $\mathrm{Yu}^{706}$, Ke Yuan ${ }^{170,493,707}$, Yuan Yuan ${ }^{9}$, Denis Yuen ${ }^{47}$, Christina K. Yung ${ }^{29}$

Olga Zaikova ${ }^{708}$, Jorge Zamora ${ }^{2,22,23,24}$, Marc Zapatka ${ }^{368}$, Jean C. Zenklusen ${ }^{166}$,

Thorsten Zenz ${ }^{149}$, Nikolajs Zeps ${ }^{709,710}$, Cheng-Zhong Zhang ${ }^{58,711}$, Fan Zhang ${ }^{470}$, Hailei Zhang ${ }^{58}$,

Hongwei Zhang ${ }^{474}$, Hongxin Zhang ${ }^{42}$, Jiashan Zhang ${ }^{166}$, Jing Zhang ${ }^{310}$, Junjun Zhang ${ }^{29}$,

Xiuqing Zhang ${ }^{300}$, Xuanping Zhang ${ }^{336,372}$, Yan Zhang ${ }^{310,712,713}$, Zemin Zhang ${ }^{349,350}$,

Zhongming Zhao ${ }^{714}$, Liangtao Zheng ${ }^{470}$, Xiuqing Zheng ${ }^{470}$, Wanding Zhou ${ }^{609}$,

Yong Zhou ${ }^{300}$, Bin Zhu ${ }^{188}$, Hongtu Zhu ${ }^{704,715}$, Jingchun Zhu' ${ }^{166}$, Shida Zhu ${ }^{300,367}$, Lihua Zou ${ }^{716}$,

Xueqing Zou ${ }^{2}$, Anna deFazio ${ }^{194,195,717}$, Nicholas van As ${ }^{167}$, Carolien H. M. van Deurzen ${ }^{718}$,

Marc J. van de Vijver ${ }^{509}$, L. van't Veer ${ }^{719}$ \& Christian von Mering ${ }^{408,720}$

${ }^{1}$ Applied Tumor Genomics Research Program, Research Programs Unit, University of Helsinki, Helsinki, Finland. ${ }^{2}$ Wellcome Sanger Institute, Wellcome Genome Campus, Hinxton, UK. ${ }^{3}$ Memorial Sloan Kettering Cancer Center, New York, NY, USA. ${ }^{4}$ Genome Science Division, Research Center for Advanced Science and Technology, University of Tokyo, Tokyo, Japan. ${ }^{5}$ Department of Surgery, University of Chicago, Chicago, IL, USA. ${ }^{6}$ Department of Surgery, Division of Hepatobiliary and Pancreatic Surgery, School of Medicine, Keimyung University Dongsan Medical Center, Daegu, South Korea. ${ }^{7}$ Department of Oncology, Gil Medical Center, Gachon University, Incheon, South Korea. ${ }^{8}$ Hiroshima University, Hiroshima, Japan.

${ }^{9}$ Department of Bioinformatics and Computational Biology, The University of Texas MD Anderson Cancer Center, Houston, TX, USA. ${ }^{10}$ University of Texas MD Anderson Cancer Center, Houston, TX, USA. "King Faisal Specialist Hospital and Research Centre, Al Maather, Riyadh, Saudi Arabia. ${ }^{12}$ Bioinformatics Unit, Spanish National Cancer Research Centre (CNIO) Madrid, Spain. ${ }^{13}$ Bioinformatics Core Facility, University Medical Center Hamburg, Hamburg, Germany. ${ }^{14}$ Heinrich Pette Institute, Leibniz Institute for Experimental Virology, Hamburg, Germany. ${ }^{15}$ Ontario Tumour Bank, Ontario Institute for Cancer Research, Toronto, ON, Canada. ${ }^{16}$ Department of Pathology, The University of Texas MD Anderson Cancer Center, Houston, TX, USA. ${ }^{17}$ Laboratory of Pathology, Center for Cancer Research, National Cancer Institute, Bethesda, MD, USA. ${ }^{18}$ Department of Cellular and Molecular Medicine and Department of Bioengineering, University of California San Diego, La Jolla, CA, USA. ${ }^{19}$ UC San Diego Moores Cancer Center, San Diego, CA, USA. ${ }^{20}$ Canada's Michael Smith Genome Sciences Centre, BC Cancer, Vancouver, BC, Canada. ${ }^{21}$ Sir Peter MacCallum Department of Oncology, Peter MacCallum Cancer Centre, University of Melbourne, Melbourne, VIC, Australia. ${ }^{22}$ Centre for Research in Molecular Medicine and Chronic Diseases (CiMUS), Universidade de Santiago de Compostela, Santiago de Compostela, Spain. ${ }^{23}$ Department of Zoology, Genetics and Physical Anthropology, (CiMUS), Universidade de Santiago de Compostela, Santiago de Compostela, Spain. ${ }^{24}$ The Biomedical Research Centre (CINBIO), Universidade de Vigo, Vigo, Spain. ${ }^{25}$ Royal National Orthopaedic Hospital - Bolsover, London, UK. ${ }^{26}$ Department of Genomic Medicine, The University of Texas MD Anderson Cancer Center, Houston, TX, USA. ${ }^{27}$ Quantitative and Computational Biosciences Graduate Program, Baylor College of Medicine, Houston, TX, USA. ${ }^{28}$ The Jackson Laboratory for Genomic Medicine, Farmington, CT, USA. ${ }^{29}$ Genome Informatics Program, Ontario Institute for Cancer Research, Toronto, ON, Canada. ${ }^{30}$ Institute of Human Genetics, Christian-Albrechts-University, Kiel, Germany. ${ }^{31}$ Institute of Human Genetics, Ulm University and Ulm University Medical Center, Ulm Germany. ${ }^{32}$ Queensland Centre for Medical Genomics, Institute for Molecular Bioscience, University of Queensland, St. Lucia, Brisbane, QLD, Australia. ${ }^{33}$ Salford Royal NHS Foundation Trust, Salford, UK. ${ }^{34}$ Department of Surgery, Pancreas Institute, University and Hospital Trust of Verona, Verona, Italy. ${ }^{35}$ Molecular and Medical Genetics, OHSU Knight Cancer Institute, Oregon Health and Science University, Portland, OR, USA. ${ }^{36}$ Department of Molecular Oncology, BC Cancer Research Centre, Vancouver, BC, Canada. ${ }^{37}$ The McDonnell Genome Institute at Washington University, St. Louis, MO, USA. ${ }^{38}$ University College London, London, UK. ${ }^{39}$ Division of Cancer Genomics, National Cancer Center Research Institute, National Cancer Center, Tokyo, Japan. ${ }^{40}$ DLR Project Management Agency, Bonn, Germany. ${ }^{41}$ Tokyo Women's Medical University, Tokyo, Japan. ${ }^{42}$ Center for Molecular Oncology, Memorial Sloan Kettering Cancer Center, New York, NY, USA. ${ }^{43}$ Los Alamos National Laboratory, Los Alamos, NM, USA. ${ }^{44}$ Department of Pathology, University Health Network, Toronto General Hospital, Toronto, ON, Canada. ${ }^{45}$ Nottingham University Hospitals NHS Trust, Nottingham, UK. ${ }^{46}$ Epigenomics and Cancer Risk Factors, German Cancer Research Center (DKFZ), Heidelberg, Germany. ${ }^{47}$ Computational Biology Program, Ontario Institute for Cancer Research, Toronto, ON, Canada. ${ }^{48}$ Department of Molecular Genetics, University of Toronto, Toronto, ON, Canada. ${ }^{49}$ Vector Institute, Toronto, ON, Canada. ${ }^{50} \mathrm{Hematopathology} \mathrm{Section,} \mathrm{Institute} \mathrm{of}$ Pathology, Christian-Albrechts-University, Kiel, Germany. ${ }^{51}$ Department of Pathology and Laboratory Medicine, School of Medicine, University of North Carolina at Chapel Hill, Chapel Hill, NC, USA. ${ }^{52}$ Department of Cancer Genetics, Institute for Cancer Research, Oslo University Hospital, The Norwegian Radium Hospital, Oslo, Norway. ${ }^{53}$ Pathology, Hospital Clinic, Institut d'Investigacions Biomèdiques August Pi i Sunyer (IDIBAPS), University of Barcelona, Barcelona, Spain. ${ }^{54}$ Department of Veterinary Medicine, Transmissible Cancer Group, University of Cambridge, Cambridge, UK. ${ }^{55}$ Alvin J. Siteman Cancer Center, Washington University School of Medicine, St. Louis, MO, USA. ${ }^{56}$ Wolfson Wohl Cancer Research Centre, Institute of Cancer Sciences, University of Glasgow, Glasgow, UK. ${ }^{57}$ Lineberger Comprehensive Cancer Center, University of North Carolina at Chapel Hill, Chapel Hill, NC, USA. ${ }^{58}$ Broad Institute of MIT and Harvard, Cambridge, MA, USA. ${ }^{59}$ Dana-Farber/Boston Children's Cancer and Blood Disorders Center, Boston, MA, USA. ${ }^{60}$ Department of Pediatrics, Harvard Medical School, Boston, MA, USA. ${ }^{61}$ Leeds Institute of Medical Research @ St. James's, University of Leeds, St. James's University Hospital, Leeds, UK. ${ }^{62}$ Department of Pathology and Diagnostics, University and Hospital Trust of Verona,
Verona, Italy ${ }^{63}$ Department of Surgery, Princess Alexandra Hospital, Brisbane, QLD, Australia. ${ }^{64}$ Surgical Oncology Group, Diamantina Institute, University of Queensland, Brisbane, QLD, Australia. ${ }^{65}$ Department of Population and Quantitative Health Sciences, Case Western Reserve University School of Medicine, Cleveland, OH, USA. ${ }^{66}$ Research Health Analytics and Informatics, University Hospitals Cleveland Medical Center, Cleveland, OH, USA. ${ }^{67}$ Gloucester Royal Hospital, Gloucester, UK. ${ }^{68}$ European Molecular Biology Laboratory, European Bioinformatics Institute (EMBL-EBI), Cambridge, UK. ${ }^{69}$ Diagnostic Development, Ontario Institute for Cancer Research, Toronto, ON, Canada. ${ }^{70}$ Barcelona Supercomputing Center (BSC), Barcelona, Spain. ${ }^{71}$ Arnie Charbonneau Cancer Institute, University of Calgary, Calgary, $A B$, Canada. ${ }^{72}$ Departments of Surgery and Oncology, University of Calgary, Calgary, $A B$, Canada. ${ }^{73}$ Department of Pathology, Oslo University Hospital, The Norwegian Radium Hospital, Oslo, Norway. ${ }^{74} \mathrm{PanCuRx}$ Translational Research Initiative, Ontario Institute for Cancer Research, Toronto, ON, Canada. ${ }^{75}$ Department of Oncology, Sidney Kimmel Comprehensive Cancer Center at Johns Hopkins University School of Medicine, Baltimore, MD, USA. ${ }^{76}$ University Hospital Southampton NHS Foundation Trust, Southampton, UK. ${ }^{77}$ Royal Stoke University Hospital, Stoke-on-Trent, UK. ${ }^{78} \mathrm{Genome} \mathrm{Sequence} \mathrm{Informatics,} \mathrm{Ontario}$ Institute for Cancer Research, Toronto, ON, Canada. ${ }^{79}$ Human Longevity Inc, San Diego, CA, USA. ${ }^{80}$ Olivia Newton-John Cancer Research Institute, La Trobe University, Heidelberg, VIC, Australia. ${ }^{81}$ Computer Network Information Center, Chinese Academy of Sciences, Beijing, China. ${ }^{82}$ Genome Canada, Ottawa, ON, Canada. ${ }^{83}$ CNAG-CRG, Centre for Genomic Regulation (CRG), Barcelona Institute of Science and Technology (BIST), Barcelona, Spain. ${ }^{84}$ Universitat Pompeu Fabra (UPF), Barcelona, Spain. ${ }^{85}$ Buck Institute for Research on Aging, Novato, CA, USA. ${ }^{86}$ Duke University Medical Center, Durham, NC, USA. ${ }^{87}$ Department of Human Genetics, Hannover Medical School, Hannover, Germany. ${ }^{88}$ Center for Bioinformatics and Functional Genomics, Cedars-Sinai Medical Center, Los Angeles, CA, USA. ${ }^{89}$ Department of Biomedical Sciences, Cedars-Sinai Medical Center, Los Angeles, CA, USA. ${ }^{90}$ The Hebrew University Faculty of Medicine, Jerusalem, Israel. ${ }^{91}$ Barts Cancer Institute, Barts and the London School of Medicine and Dentistry, Queen Mary University of London, London, UK. ${ }^{92}$ Department of Computer Science, Bioinformatics Group, University of Leipzig, Leipzig, Germany. ${ }^{93}$ Interdisciplinary Center for Bioinformatics, University of Leipzig, Leipzig, Germany. ${ }^{94}$ Transcriptome Bioinformatics, LIFE Research Center for Civilization Diseases, University of Leipzig, Leipzig, Germany. ${ }^{95}$ Department of Medical Oncology, Dana-Farber Cancer Institute, Boston, MA, USA. ${ }^{96}$ Department of Cancer Biology, Dana-Farber Cancer Institute, Boston, MA, USA. ${ }^{97}$ Harvard Medical School, Boston, MA, USA. ${ }^{98}$ USC Norris Comprehensive Cancer Center, University of Southern California, Los Angeles, CA, USA. ${ }^{99}$ Department of Diagnostics and Public Health, University and Hospital Trust of Verona, Verona, Italy. ${ }^{100}$ Department of Mathematics, Aarhus University, Aarhus, Denmark. ${ }^{101}$ Department of Molecular Medicine (MOMA), Aarhus University Hospital, Aarhus N, Denmark. ${ }^{102}$ Instituto Carlos Slim de la Salud, Mexico City, Mexico. ${ }^{103}$ Department of Medical Biophysics, University of Toronto, Toronto, ON, Canada. ${ }^{104}$ Cancer Division, Garvan Institute of Medical Research, Kinghorn Cancer Centre, University of New South Wales (UNSW Sydney), Sydney, NSW, Australia. ${ }^{105}$ South Western Sydney Clinical School, Faculty of Medicine, University of New South Wales (UNSW Sydney), Liverpool, NSW, Australia. ${ }^{106}$ West of Scotland Pancreatic Unit, Glasgow Royal Infirmary, Glasgow, UK. ${ }^{107}$ Center for Digital Health, Berlin Institute of Health and Charitè Universitätsmedizin Berlin, Berlin, Germany. ${ }^{108}$ Heidelberg Center for Personalized Oncology (DKFZ-HIPO), German Cancer Research Center (DKFZ), Heidelberg, Germany. ${ }^{109}$ The Preston Robert Tisch Brain Tumor Center, Duke University Medical Center, Durham, NC, USA. ${ }^{110}$ Massachusetts General Hospital, Boston, MA, USA. ${ }^{111}$ National Institute of Biomedical Genomics, Kalyani, West Bengal, India. ${ }^{112}$ Institute of Clinical Medicine and Institute of Oral Biology, University of Oslo, Oslo, Norway. ${ }^{113}$ University of North Carolina at Chapel Hill, Chapel Hill, NC, USA. ${ }^{114}$ ARC-Net Centre for Applied Research on Cancer, University and Hospital Trust of Verona, Verona, Italy. ${ }^{115}$ The Institute of Cancer Research, London, UK. ${ }^{116}$ Centre for Computational Biology, Duke-NUS Medical School, Singapore, Singapore. ${ }^{117}$ Programme in Cancer and Stem Cell Biology, Duke-NUS Medical School, Singapore, Singapore. ${ }^{118}$ Division of Oncology and Pathology, Department of Clinical Sciences Lund, Lund University, Lund, Sweden. ${ }^{119}$ Department of Pediatric Oncology, Hematology and Clinical Immunology, Heinrich-Heine-University, Düsseldorf, Germany. ${ }^{120}$ Laboratory for Medical Science Mathematics, RIKEN Center for Integrative Medical Sciences, Yokohama, Japan. ${ }^{121}$ RIKEN Center for Integrative Medical Sciences, Yokohama, Japan. ${ }^{122}$ Department of Internal Medicine/Hematology, Friedrich-Ebert-Hospital, Neumünster, Germany. ${ }^{123}$ Departments of Dermatology and Pathology, Yale University, New Haven, CT, USA. ${ }^{124}$ Centre for Genomic Regulation (CRG), The Barcelona Institute of Science and Technology, Barcelona, Spain. ${ }^{125}$ Radcliffe Department of Medicine, University of Oxford, Oxford, UK. ${ }^{126}$ Canadian Center for Computational Genomics, McGill University, Montreal, QC, Canada. ${ }^{127}$ Department of Human Genetics, McGill University, Montreal, QC, Canada. ${ }^{128}$ Department of Human Genetics, University of California Los Angeles, Los Angeles, CA, USA. ${ }^{129}$ Department of Pharmacology, University of Toronto, Toronto, ON, Canada. ${ }^{130}$ Faculty of Medicine and Health Technology, Tampere University and Tays Cancer Center, Tampere University Hospital, Tampere, Finland. ${ }^{131}$ Haematology, Leeds Teaching Hospitals NHS Trust, Leeds, UK. ${ }^{132}$ Translational Research and Innovation, Centre Léon Bérard, Lyon, France. ${ }^{133}$ Fox Chase Cancer Center, Philadelphia, PA, USA. ${ }^{134}$ International Agency for Research on Cancer, World Health Organization, Lyon, France. ${ }^{135}$ Earlham Institute, Norwich, UK. ${ }^{136}$ Norwich Medical School, University of East Anglia, Norwich, UK. ${ }^{137}$ Department of Molecular Biology, Faculty of Science, Radboud Institute for Molecular Life Sciences, Radboud University, Nijmegen, HB, The Netherlands. ${ }^{138}$ CRUK Manchester Institute and Centre, Manchester, UK. ${ }^{139}$ Department of Radiation Oncology, University of Toronto, Toronto, ON, Canada. ${ }^{140}$ Division of Cancer Sciences, Manchester Cancer Research Centre, University of Manchester, Manchester, UK. ${ }^{141}$ Radiation 
Medicine Program, Princess Margaret Cancer Centre, Toronto, ON, Canada. ${ }^{142}$ Department of Pathology, Brigham and Women's Hospital, Harvard Medical School, Boston, MA, USA. ${ }^{143}$ Department of Surgery, Division of Thoracic Surgery, The Johns Hopkins University School of Medicine, Baltimore, MD, USA. ${ }^{144}$ Division of Molecular Pathology, The Netherlands Cancer Institute, Oncode Institute, Amsterdam, CX, The Netherlands. ${ }^{145}$ Department of Biomolecular Engineering, University of California Santa Cruz, Santa Cruz, CA, USA. ${ }^{146}$ UC Santa Cruz Genomics Institute, University of California Santa Cruz, Santa Cruz, CA, USA. ${ }^{147}$ Division of Applied Bioinformatics, German Cancer Research Center (DKFZ), Heidelberg, Germany. ${ }^{148}$ German Cancer Consortium (DKTK), German Cancer Research Center (DKFZ), Heidelberg, Germany. ${ }^{149}$ National Center for Tumor Diseases (NCT) Heidelberg, Heidelberg, Germany. ${ }^{150} \mathrm{Center}$ for Biological Sequence Analysis, Department of Bio and Health Informatics, Technical University of Denmark, Lyngby, Denmark. ${ }^{151}$ Novo Nordisk Foundation Center for Protein Research, University of Copenhagen, Copenhagen, Denmark. ${ }^{152}$ Institute for Molecular Bioscience, University of Queensland, St. Lucia, Brisbane, QLD, Australia. ${ }^{153}$ Biomedical Engineering, Oregon Health and Science University, Portland, OR, USA. ${ }^{154}$ Division of Theoretical Bioinformatics, German Cancer Research Center (DKFZ), Heidelberg, Germany. ${ }^{155}$ Institute of Pharmacy and Molecular Biotechnology and BioQuant, Heidelberg University, Heidelberg, Germany. ${ }^{156}$ Federal Ministry of Education and Research, Berlin, Germany. ${ }^{157}$ Melanoma Institute Australia, University of Sydney, Sydney, NSW, Australia. ${ }^{158}$ Pediatric Hematology and Oncology, University Hospital Muenster, Muenster, Germany. ${ }^{159}$ Department of Pathology, Johns Hopkins University School of Medicine, Baltimore, MD, USA. ${ }^{160}$ McKusick-Nathans Institute of Genetic Medicine, Sidney Kimmel Comprehensive Cancer Center at Johns Hopkins University School of Medicine, Baltimore, MD, USA. ${ }^{161}$ Foundation Medicine, Inc, Cambridge, MA, USA. ${ }^{162}$ Department of Biomedical Data Science, Stanford University School of Medicine, Stanford, CA, USA. ${ }^{163}$ Department of Genetics, Stanford University School of Medicine, Stanford, CA, USA. ${ }^{164}$ Bakar Computational Health Sciences Institute and Department of Pediatrics, University of California, San Francisco, CA, USA. ${ }^{165}$ Institute of Clinical Medicine, Faculty of Medicine, University of Oslo, Oslo, Norway. ${ }^{166} \mathrm{National}$ Cancer Institute, National Institutes of Health, Bethesda, MD, USA. ${ }^{167}$ Royal Marsden NHS Foundation Trust, London and Sutton, UK. ${ }^{168} \mathrm{Genome} \mathrm{Biology} \mathrm{Unit,} \mathrm{European}$ Molecular Biology Laboratory (EMBL), Heidelberg, Germany. ${ }^{169}$ Department of Oncology, University of Cambridge, Cambridge, UK. ${ }^{70} \mathrm{Li}$ Ka Shing Centre, Cancer Research UK Cambridge Institute, University of Cambridge, Cambridge, UK. ${ }^{171}$ Institut Gustave Roussy, Villejuif, France. ${ }^{172}$ Cambridge University Hospitals NHS Foundation Trust, Cambridge, UK. ${ }^{173}$ Department of Haematology, University of Cambridge, Cambridge, UK. ${ }^{174}$ Anatomia Patológica, Hospital Clinic, Institut d'Investigacions Biomèdiques August Pi i Sunyer (IDIBAPS), University of Barcelona, Barcelona, Spain. ${ }^{175}$ Spanish Ministry of Science and Innovation, Madrid, Spain. ${ }^{176}$ University of Michigan Comprehensive Cancer Center, Ann Arbor, MI, USA. ${ }^{177}$ Department for BioMedical Research, University of Bern, Bern, Switzerland. ${ }^{178}$ Department of Medical Oncology, Inselspital, University Hospital and University of Bern, Bern, Switzerland. ${ }^{179}$ Graduate School for Cellular and Biomedical Sciences, University of Bern, Bern, Switzerland. ${ }^{180}$ University of Pavia, Pavia, Italy. ${ }^{181}$ University of Alabama at Birmingham, Birmingham, AL, USA. ${ }^{182} \mathrm{UHN}$ Program in BioSpecimen Sciences, Toronto General Hospital, Toronto, ON, Canada. ${ }^{183}$ Department of Urology, Icahn School of Medicine at Mount Sinai, New York, NY, USA. ${ }^{184}$ Centre for Law and Genetics, University of Tasmania, Sandy Bay Campus, Hobart, TAS, Australia. ${ }^{185}$ Faculty of Biosciences, Heidelberg University, Heidelberg, Germany. ${ }^{186}$ Department of Biochemistry, Microbiology and Immunology, Faculty of Medicine, University of Ottawa, Ottawa, ON, Canada. ${ }^{187}$ Division of Anatomic Pathology, Mayo Clinic, Rochester, MN, USA. ${ }^{188}$ Division of Cancer Epidemiology and Genetics, National Cancer Institute, National Institutes of Health, Bethesda, MD, USA. ${ }^{189}$ Illawarra Shoalhaven Local Health District L3 Illawarra Cancer Care Centre, Wollongong Hospital, Wollongong, NSW, Australia. ${ }^{190}$ BioForA, French National Institute for Agriculture, Food, and Environment (INRAE), ONF, Orléans, France. ${ }^{191}$ Department of Biostatistics, Bloomberg School of Public Health, Johns Hopkins University, Baltimore, MD, USA. ${ }^{192}$ University of California San Diego, San Diego, CA, USA. ${ }^{193}$ Division of Experimental Pathology, Mayo Clinic, Rochester, MN, USA. ${ }^{194}$ Centre for Cancer Research, The Westmead Institute for Medical Research, University of Sydney, Sydney, NSW, Australia. ${ }^{195}$ Department of Gynaecological Oncology, Westmead Hospital, Sydney, NSW, Australia. ${ }^{196} \mathrm{PDX}$ en Biosystems Inc, Seoul, South Korea. ${ }^{197} \mathrm{Korea}$ Advanced Institute of Science and Technology, Daejeon, South Korea. ${ }^{198}$ Electronics and Telecommunications Research Institute, Daejeon, South Korea. ${ }^{199}$ Institut National du Cancer (INCA), Boulogne-Billancourt, France. ${ }^{200}$ Department of Genetics, Informatics Institute, University of Alabama at Birmingham, Birmingham, AL, USA. ${ }^{201}$ Division of Medical Oncology, National Cancer Centre, Singapore, Singapore. ${ }^{202} \mathrm{Medical}$ Oncology, University and Hospital Trust of Verona, Verona, Italy. ${ }^{203}$ Department of Pediatrics, University Hospital Schleswig-Holstein, Kiel, Germany. ${ }^{204} \mathrm{Hepatobiliary/Pancreatic} \mathrm{Surgical} \mathrm{Oncology} \mathrm{Program,}$ University Health Network, Toronto, ON, Canada. ${ }^{205}$ School of Biological Sciences, University of Auckland, Auckland, New Zealand. ${ }^{206}$ Department of Surgery, University of Melbourne, Parkville, VIC, Australia. ${ }^{207}$ The Murdoch Children's Research Institute, Royal Children's Hospital, Parkville, VIC, Australia. ${ }^{208}$ Walter and Eliza Hall Institute, Parkville, VIC, Australia. ${ }^{209}$ Vancouver Prostate Centre, Vancouver, Canada. ${ }^{210}$ Lunenfeld-Tanenbaum Research Institute, Mount Sinai Hospital, Toronto, ON, Canada. ${ }^{211}$ University of East Anglia, Norwich, UK. ${ }^{212}$ Norfolk and Norwich University Hospital NHS Trust, Norwich, UK. ${ }^{213}$ Victorian Institute of Forensic Medicine, Southbank, VIC, Australia. ${ }^{214}$ Department of Biomedical Informatics, Harvard Medical School, Boston, MA, USA. ${ }^{215}$ Department of Chemistry, Centre for Molecular Science Informatics, University of Cambridge, Cambridge, UK. ${ }^{216}$ Ludwig Center at Harvard Medical School, Boston, MA, USA. ${ }^{27} \mathrm{Human}$ Genome Sequencing Center, Baylor College of Medicine, Houston, TX, USA. ${ }^{218}$ Peter MacCallum Cancer Centre, University of Melbourne,
Melbourne, VIC, Australia. ${ }^{219}$ Physics Division, Optimization and Systems Biology Lab, Massachusetts General Hospital, Boston, MA, USA. ${ }^{220}$ Department of Medicine, Baylor College of Medicine, Houston, TX, USA. ${ }^{22}$ University of Cologne, Cologne, Germany. ${ }^{222}$ International Genomics Consortium, Phoenix, AZ, USA. ${ }^{223}$ Genomics Research Program, Ontario Institute for Cancer Research, Toronto, ON, Canada. ${ }^{224}$ Barking Havering and Redbridge University Hospitals NHS Trust, Romford, UK. ${ }^{225}$ Children's Hospital at Westmead, University of Sydney, Sydney, NSW, Australia. ${ }^{226}$ Department of Medicine, Section of Endocrinology, University and Hospital Trust of Verona, Verona, Italy. ${ }^{227}$ Computational Biology Center, Memorial Sloan Kettering Cancer Center, New York, NY, USA. ${ }^{228}$ Department of Biology, ETH Zurich, Zürich, Switzerland. ${ }^{229}$ Department of Computer Science, ETH Zurich, Zurich, Switzerland. ${ }^{230} \mathrm{SIB}$ Swiss Institute of Bioinformatics, Lausanne, Switzerland. ${ }^{231}$ Weill Cornell Medical College, New York, NY, USA. ${ }^{232}$ Academic Department of Medical Genetics, University of Cambridge, Addenbrooke's Hospital, Cambridge, UK. ${ }^{233} \mathrm{MRC}$ Cancer Unit, University of Cambridge, Cambridge, UK. ${ }^{234}$ Departments of Pediatrics and Genetics, University of North Carolina at Chapel Hill, Chapel Hill, NC, USA. ${ }^{235}$ Seven Bridges Genomics, Charlestown, MA, USA. ${ }^{236}$ Annai Systems, Inc, Carlsbad, CA, USA. ${ }^{27}$ Department of Pathology, General Hospital of Treviso, Department of Medicine, University of Padua, Treviso, Italy. ${ }^{238}$ Department of Computational Biology, University of Lausanne, Lausanne, Switzerland. ${ }^{239}$ Department of Genetic Medicine and Development, University of Geneva Medical School, Geneva, CH, Switzerland. ${ }^{240}$ Swiss Institute of Bioinformatics, University of Geneva, Geneva, $\mathrm{CH}$, Switzerland. ${ }^{241}$ The Francis Crick Institute, London, UK. ${ }^{242}$ University of Leuven, Leuven, Belgium. ${ }^{243}$ Institute of Medical Genetics and Applied Genomics, University of Tübingen, Tübingen, Germany. ${ }^{244}$ Computational and Systems Biology, Genome Institute of Singapore, Singapore, Singapore. ${ }^{245}$ School of Computing, National University of Singapore, Singapore, Singapore. ${ }^{246} \mathrm{Big}$ Data Institute, Li Ka Shing Centre, University of Oxford, Oxford, UK. ${ }^{247}$ Biomedical Data Science Laboratory, Francis Crick Institute, London, UK. ${ }^{248}$ Bioinformatics Group, Department of Computer Science, University College London, London, UK. ${ }^{249}$ The Edward S. Rogers Sr. Department of Electrical and Computer Engineering, University of Toronto, Toronto, ON, Canada. ${ }^{250}$ Breast Cancer Translational Research Laboratory JC Heuson, Institut Jules Bordet, Brussels, Belgium. ${ }^{251}$ Department of Oncology, Laboratory for Translational Breast Cancer Research, KU Leuven, Leuven, Belgium. ${ }^{252}$ Institute for Research in Biomedicine (IRB Barcelona), The Barcelona Institute of Science and Technology, Barcelona, Spain. ${ }^{253}$ Research Program on Biomedical Informatics, Universitat Pompeu Fabra, Barcelona, Spain. ${ }^{254}$ Division of Medical Oncology, Princess Margaret Cancer Centre, Toronto, ON, Canada. ${ }^{255}$ Department of Physiology and Biophysics, Weill Cornell Medicine, New York, NY, USA. ${ }^{256}$ Institute for Computational Biomedicine, Weill Cornell Medicine, New York, NY, USA. ${ }^{257}$ Department of Pathology, UPMC Shadyside, Pittsburgh, PA, USA. ${ }^{258}$ Independent Consultant, Wellesley, USA. ${ }^{259}$ Department of Cell and Molecular Biology, Science for Life Laboratory, Uppsala University, Uppsala, Sweden. ${ }^{260}$ Department of Medicine and Department of Genetics, Washington University School of Medicine, St. Louis, St. Louis, MO, USA. ${ }^{261} \mathrm{Hefei}$ University of Technology, Anhui, China. ${ }^{262}$ Translational Cancer Research Unit, GZA Hospitals St.-Augustinus, Center for Oncological Research, Faculty of Medicine and Health Sciences, University of Antwerp, Antwerp, Belgium. ${ }^{263}$ Simon Fraser University, Burnaby, BC, Canada. ${ }^{264}$ University of Pennsylvania, Philadelphia, PA, USA. ${ }^{265}$ Faculty of Science and Technology, University of Vic-Central University of Catalonia (UVic-UCC), Vic, Spain. ${ }^{266}$ The Wellcome Trust, London, UK. ${ }^{267}$ The Hospital for Sick Children, Toronto, ON, Canada. ${ }^{268}$ Department of Pathology, Queen Elizabeth University Hospital, Glasgow, UK. ${ }^{269}$ Department of Genetics and Computational Biology, QIMR Berghofer Medical Research Institute, Brisbane, QLD, Australia. ${ }^{270}$ Department of Oncology, Centre for Cancer Genetic Epidemiology, University of Cambridge, Cambridge, UK. ${ }^{271}$ Department of Public Health and Primary Care, Centre for Cancer Genetic Epidemiology, University of Cambridge, Cambridge, UK. ${ }^{272}$ Prostate Cancer Canada, Toronto, ON, Canada. ${ }^{273}$ University of Cambridge, Cambridge, UK. ${ }^{274}$ Department of Laboratory Medicine, Translational Cancer Research, Lund University Cancer Center at Medicon Village, Lund University, Lund, Sweden. ${ }^{275}$ Heidelberg University, Heidelberg, Germany. ${ }^{276} \mathrm{New}$ BIH Digital Health Center, Berlin Institute of Health (BIH) and CharitéUniversitätsmedizin Berlin, Berlin, Germany. ${ }^{277} \mathrm{CIBER}$ Epidemiología y Salud Pública (CIBERESP), Madrid, Spain. ${ }^{278}$ Research Group on Statistics, Econometrics and Health (GRECS), UdG, Barcelona, Spain. ${ }^{279}$ Quantitative Genomics Laboratories (qGenomics), Barcelona, Spain. ${ }^{280}$ Icelandic Cancer Registry, Icelandic Cancer Society, Reykjavik, Iceland. ${ }^{281}$ State Key Laboratory of Cancer Biology, and Xijing Hospital of Digestive Diseases, Fourth Military Medical University, Shaanxi, China. ${ }^{282}$ Department of Medicine (DIMED), Surgical Pathology Unit, University of Padua, Padua, Italy. ${ }^{283}$ Rigshospitalet, Copenhagen, Denmark. ${ }^{284}$ Center for Cancer Genomics, National Cancer Institute, National Institutes of Health, Bethesda, MD, USA. ${ }^{285}$ Department of Biochemistry and Molecular Medicine, University of Montreal, Montreal, QC, Canada. ${ }^{286}$ Australian Institute of Tropical Health and Medicine, James Cook University, Douglas, QLD, Australia. ${ }^{287}$ Department of Neuro-Oncology, Istituto Neurologico Besta, Milano, Italy. ${ }^{288}$ Bioplatforms Australia, North Ryde, NSW, Australia. ${ }^{289}$ Department of Pathology (Research), University College London Cancer Institute, London, UK. ${ }^{290}$ Department of Surgical Oncology, Princess Margaret Cancer Centre, Toronto, ON, Canada. ${ }^{29}$ Department of Medical Oncology, Josephine Nefkens Institute and Cancer Genomics Centre, Erasmus Medical Center, Rotterdam, CN, The Netherlands. ${ }^{292}$ The University of Queensland Thoracic Research Centre, The Prince Charles Hospital, Brisbane, QLD, Australia. ${ }^{293} \mathrm{CIBIO} / \mathrm{InBIO}$ - Research Center in Biodiversity and Genetic Resources, Universidade do Porto, Vairão, Portugal. ${ }^{294} \mathrm{HCA}$ Laboratories, London, UK. ${ }^{295}$ University of Liverpool, Liverpool, UK. ${ }^{296}$ The Azrieli Faculty of Medicine, Bar-Ilan University, Safed, Israel. ${ }^{297}$ Department of Neurosurgery, University of Florida, Gainesville, FL, USA. ${ }^{298}$ Department of Pathology, Graduate School of Medicine, University of Tokyo, Tokyo, Japan. ${ }^{299}$ University of 


\section{Article}

Milano Bicocca, Monza, Italy. ${ }^{300} \mathrm{BGI}$-Shenzhen, Shenzhen, China. ${ }^{301}$ Department of Pathology, Oslo University Hospital Ulleval, Oslo, Norway. ${ }^{302} \mathrm{Center}$ for Biomedical Informatics, Harvard Medical School, Boston, MA, USA. ${ }^{303}$ Department Biochemistry and Molecular Biomedicine, University of Barcelona, Barcelona, Spain. ${ }^{304} \mathrm{Office}$ of Cancer Genomics, National Cancer Institute, National Institutes of Health, Bethesda, MD, USA. ${ }^{305}$ Cancer Epigenomics, German Cancer Research Center (DKFZ), Heidelberg, Germany. ${ }^{306}$ Department of Cancer Biology, The University of Texas MD Anderson Cancer Center, Houston, TX, USA. ${ }^{307}$ Department of Surgical Oncology, The University of Texas MD Anderson Cancer Center, Houston, TX, USA. ${ }^{308}$ Department of Computer Science, Yale University, New Haven, CT, USA. ${ }^{309}$ Department of Molecular Biophysics and Biochemistry, Yale University, New Haven, CT, USA. ${ }^{310}$ Program in Computational Biology and Bioinformatics, Yale University, New Haven, CT, USA. ${ }^{311}$ Center for Cancer Research, Massachusetts General Hospital, Boston, MA, USA. ${ }^{312}$ Department of Pathology, Massachusetts General Hospital, Boston, MA, USA. ${ }^{313}$ Department of Pathology, Memorial Sloan Kettering Cancer Center, New York, NY, USA. ${ }^{31}$ Division of Gastroenterology and Hepatology, Mayo Clinic, Rochester, MN, USA. ${ }^{315}$ University of Sydney, Sydney, NSW, Australia. ${ }^{316}$ University of Oxford, Oxford, UK. ${ }^{37}$ Department of Surgery, Academic Urology Group, University of Cambridge, Cambridge, UK. ${ }^{318}$ Department of Medicine II, University of Würzburg, Wuerzburg, Germany. ${ }^{319}$ Sylvester Comprehensive Cancer Center, University of Miami, Miami, FL, USA. ${ }^{320}$ Institut Hospital del Mar d'Investigacions Mèdiques (IMIM), Barcelona, Spain. ${ }^{32} \mathrm{G}$ Genome Integrity and Structural Biology Laboratory, National Institute of Environmental Health Sciences (NIEHS), Durham, NC, USA. ${ }^{322}$ St. Thomas's Hospital, London, UK. ${ }^{323}$ Osaka International Cancer Center, Osaka, Japan. ${ }^{324}$ Department of Pathology, Skåne University Hospital, Lund University, Lund, Sweden. ${ }^{325}$ Department of Medical Oncology, Beatson West of Scotland Cancer Centre, Glasgow, UK. ${ }^{326}$ National Human Genome Research Institute, National Institutes of Health, Bethesda, MD, USA. ${ }^{327}$ Centre for Cancer Research, Victorian Comprehensive Cancer Centre, University of Melbourne, Melbourne, VIC, Australia. ${ }^{328}$ Department of Medicine, Section of Hematology/Oncology, University of Chicago, Chicago, IL, USA. ${ }^{329}$ German Center for Infection Research (DZIF), Partner Site HamburgBorstel-Lübeck-Riems, Hamburg, Germany. ${ }^{330}$ Bioinformatics Research Centre (BiRC), Aarhus University, Aarhus, Denmark. ${ }^{331}$ Department of Biotechnology, Ministry of Science and Technology, Government of India, New Delhi, Delhi, India. ${ }^{332}$ National Cancer Centre Singapore, Singapore, Singapore. ${ }^{333}$ Brandeis University, Waltham, MA, USA. ${ }^{334}$ Department of Urologic Sciences, University of British Columbia, Vancouver, BC, Canada. ${ }^{335}$ Department of Internal Medicine, Stanford University, Stanford, CA, USA. ${ }^{336}$ The University of Texas Health Science Center at Houston, Houston, TX, USA. ${ }^{337}$ Imperial College NHS Trust, Imperial College, London, INY, UK. ${ }^{338}$ Senckenberg Institute of Pathology, University of Frankfurt Medical School, Frankfurt, Germany. ${ }^{339}$ Department of Medicine, Division of Biomedical Informatics, UC San Diego School of Medicine, San Diego, CA, USA. ${ }^{340}$ Center for Precision Health, School of Biomedical Informatics, The University of Texas Health Science Center, Houston, TX, USA. ${ }^{341} \mathrm{Oxford}$ Nanopore Technologies, New York, NY, USA. ${ }^{342}$ Institute of Medical Science, University of Tokyo, Tokyo, Japan. ${ }^{343}$ Howard Hughes Medical Institute, University of California Santa Cruz, Santa Cruz, CA, USA. ${ }^{344}$ Wakayama Medical University, Wakayama, Japan. ${ }^{345}$ Department of Internal Medicine, Division of Medical Oncology, Lineberger Comprehensive Cancer Center, University of North Carolina at Chapel Hill, Chapel Hill, NC, USA. ${ }^{346}$ University of Tennessee Health Science Center for Cancer Research, Memphis, TN, USA. ${ }^{347}$ Department of Histopathology, Salford Royal NHS Foundation Trust, Salford, UK. ${ }^{348}$ Faculty of Biology, Medicine and Health, University of Manchester, Manchester, UK. ${ }^{349} \mathrm{BIOPIC}$, ICG and College of Life Sciences, Peking University, Beijing, China. ${ }^{350}$ Peking-Tsinghua Center for Life Sciences, Peking University, Beijing, China. ${ }^{351} \mathrm{Children's}$ Hospital of Philadelphia, Philadelphia, PA, USA. ${ }^{352}$ Department of Bioinformatics and Computational Biology and Department of Systems Biology, The University of Texas MD Anderson Cancer Center, Houston, TX, USA. ${ }^{353}$ Karolinska Institute, Stockholm, Sweden. ${ }^{354}$ The Donnelly Centre, University of Toronto, Toronto, ON, Canada. ${ }^{355}$ Department of Medical Genetics, College of Medicine, Hallym University, Chuncheon, South Korea. ${ }^{356}$ Department of Experimental and Health Sciences, Institute of Evolutionary Biology (UPF-CSIC), Universitat Pompeu Fabra, Barcelona, Spain. ${ }^{357} \mathrm{Health}$ Data Science Unit, University Clinics, Heidelberg, Germany. ${ }^{358}$ Massachusetts General Hospital Center for Cancer Research, Charlestown, MA, USA. ${ }^{359}$ Hokkaido University, Sapporo, Japan. ${ }^{360}$ Department of Pathology and Clinical Laboratory, National Cancer Center Hospital, Tokyo, Japan. ${ }^{361}$ Department of Genetics, University of North Carolina at Chapel Hill, Chapel Hill, NC, USA. ${ }^{362}$ Computational Biology, Leibniz Institute on Aging - Fritz Lipmann Institute (FLI), Jena, Germany. ${ }^{363}$ University of Melbourne Centre for Cancer Research, Melbourne, VIC, Australia. ${ }^{364}$ University of Nebraska Medical Center, Omaha, NE, USA. ${ }^{365}$ Syntekabio Inc, Daejeon, South Korea. ${ }^{366}$ Department of Pathology, Academic Medical Center, Amsterdam, AZ, The Netherlands. ${ }^{367}$ China National GeneBank-Shenzhen, Shenzhen, China. ${ }^{368}$ Division of Molecular Genetics, German Cancer Research Center (DKFZ), Heidelberg, Germany. ${ }^{369}$ Division of Life Science and Applied Genomics Center, Hong Kong University of Science and Technology, Clear Water Bay, Hong Kong, China. ${ }^{370}$ Icahn School of Medicine at Mount Sinai, New York, NY, USA. ${ }^{371}$ Geneplus-Shenzhen, Shenzhen, China. ${ }^{372}$ School of Computer Science and Technology, Xi'an Jiaotong University, Xi'an, China. ${ }^{373} \mathrm{AbbVie}$, North Chicago, IL, USA. ${ }^{374}$ Institute of Pathology, Charité - University Medicine Berlin, Berlin, Germany. ${ }^{375}$ Centre for Translational and Applied Genomics, British Columbia Cancer Agency, Vancouver, BC, Canada. ${ }^{376}$ Edinburgh Royal Infirmary, Edinburgh, UK. ${ }^{37}$ Berlin Institute for Medical Systems Biology, Max Delbrück Center for Molecular Medicine, Berlin, Germany. ${ }^{378}$ Department of Pediatric Immunology, Hematology and Oncology, University Hospital, Heidelberg, Germany. ${ }^{379}$ German Cancer Research Center (DKFZ), Heidelberg, Germany. ${ }^{380}$ Heidelberg Institute for Stem Cell Technology and Experimental Medicine (HI-STEM), Heidelberg, Germany.
${ }^{38}$ Institute for Computational Biomedicine, Weill Cornell Medical College, New York, NY, USA. ${ }^{382}$ New York Genome Center, New York, NY, USA. ${ }^{383}$ Department of Urology, James Buchanan Brady Urological Institute, Johns Hopkins University School of Medicine, Baltimore, MD, USA. ${ }^{384}$ Department of Preventive Medicine, Graduate School of Medicine, The University of Tokyo, Tokyo, Japan. ${ }^{385}$ Department of Molecular and Cellular Biology, Baylor College of Medicine, Houston, TX, USA. ${ }^{386}$ Department of Pathology and Immunology, Baylor College of Medicine, Houston, TX, USA. ${ }^{37}$ Michael E. DeBakey Veterans Affairs Medical Center, Houston, TX, USA. ${ }^{388}$ Technical University of Denmark, Lyngby, Denmark. ${ }^{389}$ Department of Pathology, College of Medicine, Hanyang University, Seoul, South Korea. ${ }^{390}$ Academic Unit of Surgery, School of Medicine, College of Medical, Veterinary and Life Sciences, University of Glasgow, Glasgow Royal Infirmary, Glasgow, UK. ${ }^{391}$ Department of Pathology, Asan Medical Center, College of Medicine, Ulsan University, Songpa-gu, Seoul, South Korea. ${ }^{392}$ Science Writer, Garrett Park, MD, USA. ${ }^{393}$ International Cancer Genome Consortium (ICGC)/ICGC Accelerating Research in Genomic Oncology (ARGO) Secretariat, Ontario Institute for Cancer Research, Toronto, ON, Canada. ${ }^{394}$ University of Ljubljana, Ljubljana, Slovenia. ${ }^{395}$ Department of Public Health Sciences, University of Chicago, Chicago, IL, USA. ${ }^{396}$ Research Institute, NorthShore University HealthSystem, Evanston, IL, USA. ${ }^{397}$ Department for Biomedical Research, University of Bern, Bern, Switzerland. ${ }^{398}$ Centre of Genomics and Policy, McGill University and Génome Québec Innovation Centre, Montreal, QC, Canada. ${ }^{399}$ Carolina Center for Genome Sciences, University of North Carolina at Chapel Hill, Chapel Hill, NC, USA. ${ }^{400} \mathrm{Hopp}$ Children's Cancer Center (KiTZ), Heidelberg, Germany. ${ }^{401}$ Pediatric Glioma Research Group, German Cancer Research Center (DKFZ), Heidelberg, Germany. ${ }^{402}$ Cancer Research UK, London, UK. ${ }^{403}$ Indivumed GmbH, Hamburg, Germany. ${ }^{404}$ Genome Integration Data Center, Syntekabio, Inc, Daejeon, South Korea. ${ }^{405}$ University Hospital Zurich, Zurich, Switzerland. ${ }^{406} \mathrm{Clinical}$ Bioinformatics, Swiss Institute of Bioinformatics, Geneva, Switzerland. ${ }^{407}$ Institute for Pathology and Molecular Pathology, University Hospital Zurich, Zurich, Switzerland. ${ }^{408}$ Institute of Molecular Life Sciences, University of Zurich, Zurich, Switzerland. ${ }^{409}$ MRC Human Genetics Unit, MRC IGMM, University of Edinburgh, Edinburgh, UK. ${ }^{410}$ Women's Cancer Program at the Samuel Oschin Comprehensive Cancer Institute, Cedars-Sinai Medical Center, Los Angeles, CA, USA. ${ }^{41}$ Department of Biology, Bioinformatics Group, Division of Molecular Biology, Faculty of Science, University of Zagreb, Zagreb, Croatia. ${ }^{412}$ Department for Internal Medicine II, University Hospital Schleswig-Holstein, Kiel, Germany. ${ }^{413}$ Genetics and Molecular Pathology, SA Pathology, Adelaide, SA, Australia. ${ }^{414}$ Department of Gastric Surgery, National Cancer Center Hospital, Tokyo, Japan. ${ }^{415}$ Department of Bioinformatics, Division of Cancer Genomics, National Cancer Center Research Institute, Tokyo, Japan. ${ }^{416}$ A.A. Kharkevich Institute of Information Transmission Problems, Moscow, Russia. ${ }^{417}$ Oncology and Immunology, Dmitry Rogachev National Research Center of Pediatric Hematology, Moscow, Russia. ${ }^{418}$ Skolkovo Institute of Science and Technology, Moscow, Russia. ${ }^{419}$ Department of Surgery, The George Washington University, School of Medicine and Health Science, Washington, DC, USA. ${ }^{420}$ Endocrine Oncology Branch, Center for Cancer Research, National Cancer Institute, National Institutes of Health, Bethesda, MD, USA. ${ }^{421}$ Melanoma Institute Australia, Macquarie University, Sydney, NSW, Australia. ${ }^{422}$ MIT Computer Science and Artificial Intelligence Laboratory, Massachusetts Institute of Technology, Cambridge, MA, USA. ${ }^{423}$ Tissue Pathology and Diagnostic Oncology, Royal Prince Alfred Hospital, Sydney, NSW, Australia. ${ }^{424}$ Cholangiocarcinoma Screening and Care Program and Liver Fluke and Cholangiocarcinoma Research Centre, Faculty of Medicine, Khon Kaen University, Khon Kaen, Thailand. ${ }^{425}$ Controlled Department and Institution, New York, NY, USA. ${ }^{426}$ Englander Institute for Precision Medicine, Weill Cornell Medicine, New York, NY, USA. ${ }^{427} \mathrm{National}$ Cancer Center, Gyeonggi, South Korea. ${ }^{428}$ Department of Biochemistry, College of Medicine, Ewha Womans University, Seoul, South Korea. ${ }^{429} \mathrm{Health}$ Sciences Department of Biomedical Informatics, University of California San Diego, La Jolla, CA, USA. ${ }^{430}$ Research Core Center, National Cancer Centre Korea, Goyang-si, South Korea. ${ }^{431}$ Department of Health Sciences and Technology, Sungkyunkwan University School of Medicine, Seoul, South Korea. ${ }^{432}$ Samsung Genome Institute, Seoul, South Korea. ${ }^{433}$ Breast Oncology Program, Dana-Farber/ Brigham and Women's Cancer Center, Boston, MA, USA. ${ }^{434}$ Department of Surgery, Memorial Sloan Kettering Cancer Center, New York, NY, USA. ${ }^{435}$ Division of Breast Surgery, Brigham and Women's Hospital, Boston, MA, USA. ${ }^{436}$ Integrative Bioinformatics Support Group, National Institute of Environmental Health Sciences (NIEHS), Durham, NC, USA. ${ }^{437}$ Department of Clinical Science, University of Bergen, Bergen, Norway. ${ }^{438}$ Center For Medical Innovation, Seoul National University Hospital, Seoul, South Korea. ${ }^{439}$ Department of Internal Medicine, Seoul National University Hospital, Seoul, South Korea. ${ }^{440}$ Institute of Computer Science, Polish Academy of Sciences, Warsawa, Poland. ${ }^{41}$ Functional and Structural Genomics, German Cancer Research Center (DKFZ), Heidelberg, Germany. ${ }^{442}$ Laboratory of Translational Genomics, Division of Cancer Epidemiology and Genetics, National Cancer Institute, , National Institutes of Health, Bethesda, MD, USA. ${ }^{43}$ Institute for Medical Informatics Statistics and Epidemiology, University of Leipzig, Leipzig, Germany. ${ }^{44}$ Morgan Welch Inflammatory Breast Cancer Research Program and Clinic, The University of Texas MD Anderson Cancer Center, Houston, TX, USA. ${ }^{445}$ Department of Hematology and Oncology, Georg-AugustsUniversity of Göttingen, Göttingen, Germany. ${ }^{446}$ Institute of Cell Biology (Cancer Research), University of Duisburg-Essen, Essen, Germany. ${ }^{447}$ King's College London and Guy's and St. Thomas' NHS Foundation Trust, London, UK. ${ }^{448}$ Center for Epigenetics, Van Andel Research Institute, Grand Rapids, MI, USA. ${ }^{449}$ The University of Queensland Centre for Clinical Research, Royal Brisbane and Women's Hospital, Herston, QLD, Australia. ${ }^{450}$ Department of Pediatric Oncology and Hematology, University of Cologne, Cologne, Germany. ${ }^{45}$ University of Düsseldorf, Düsseldorf, Germany. ${ }^{452}$ Department of Pathology, Institut Jules Bordet, Brussels, Belgium. ${ }^{453}$ Institute of Biomedicine, Sahlgrenska Academy at University of Gothenburg, Gothenburg, Sweden. ${ }^{454}$ Children's Medical Research Institute, Sydney, NSW, 
Australia. ${ }^{455}$ ILSbio, LLC Biobank, Chestertown, MD, USA. ${ }^{456}$ Division of Genetics and Genomics, Boston Children's Hospital, Harvard Medical School, Boston, MA, USA. ${ }^{457}$ Institute for Bioengineering and Biopharmaceutical Research (IBBR), Hanyang University, Seoul, South Korea. ${ }^{458}$ Department of Statistics, University of California Santa Cruz, Santa Cruz, CA, USA. ${ }^{459}$ National Genotyping Center, Institute of Biomedical Sciences, Academia Sinica, Taipei, Taiwan. ${ }^{460}$ Department of Vertebrate Genomics/Otto Warburg Laboratory Gene Regulation and Systems Biology of Cancer, Max Planck Institute for Molecular Genetics, Berlin, Germany. ${ }^{461}$ McGill University and Genome Quebec Innovation Centre, Montreal, QC, Canada. ${ }^{462}$ biobyte solutions $\mathrm{GmbH}$, Heidelberg, Germany. ${ }^{463}$ Gynecologic Oncology, NYU Laura and Isaac Perlmutter Cancer Center, New York University, New York, NY, USA. ${ }^{464}$ Division of Oncology, Stem Cell Biology Section, Washington University School of Medicine, St. Louis, MO, USA. ${ }^{465}$ Department of Systems Biology, The University of Texas MD Anderson Cancer Center, Houston, TX, USA. ${ }^{466}$ Harvard University, Cambridge, MA, USA. ${ }^{467}$ Urologic Oncology Branch, Center for Cancer Research, National Cancer Institute, National Institutes of Health, Bethesda, MD, USA. ${ }^{468}$ University of Oslo, Oslo, Norway. ${ }^{469}$ University of Toronto, Toronto, ON, Canada. ${ }^{470}$ Peking University, Beijing, China. ${ }^{471}$ School of Life Sciences, Peking University, Beijing, China. ${ }^{472}$ Leidos Biomedical Research, Inc, McLean, VA, USA. ${ }^{473}$ Hematology, Hospital Clinic, Institut d'Investigacions Biomèdiques August Pi i Sunyer (IDIBAPS), University of Barcelona, Barcelona, Spain. ${ }^{474}$ Second Military Medical University, Shanghai, China. ${ }^{475}$ Chinese Cancer Genome Consortium, Shenzhen, China. ${ }^{476}$ Department of Medical Oncology, Beijing Hospital, Beijing, China. ${ }^{477}$ Laboratory of Molecular Oncology, Key Laboratory of Carcinogenesis and Translational Research (Ministry of Education), Peking University Cancer Hospital and Institute, Beijing, China. ${ }^{478}$ School of Medicine/School of Mathematics and Statistics, University of St. Andrews, St, Andrews, Fife, UK. ${ }^{479}$ Institute for Systems Biology, Seattle, WA, USA. ${ }^{480}$ Department of Biochemistry and Molecular Biology, Faculty of Medicine, University Institute of Oncology-IUOPA, Oviedo, Spain. ${ }^{481}$ Institut Bergonié, Bordeaux, France. ${ }^{482}$ Cancer Unit, MRC University of Cambridge, Cambridge, UK. ${ }^{483}$ Department of Pathology and Laboratory Medicine, Center for Personalized Medicine, Children's Hospital Los Angeles, Los Angeles, CA, USA. ${ }^{484} \mathrm{~J}$ John Curtin School of Medical Research, Canberra, ACT, Australia. ${ }^{485} \mathrm{MVZ}$ Department of Oncology, PraxisClinic am Johannisplatz, Leipzig, Germany. ${ }^{486}$ Department of Information Technology, Ghent University, Ghent, Belgium. ${ }^{487}$ Department of Plant Biotechnology and Bioinformatics, Ghent University, Ghent, Belgium. ${ }^{488}$ Institute for Genomic Medicine, Nationwide Children's Hospital, Columbus, OH, USA. ${ }^{489}$ Computational Biology Program, School of Medicine, Oregon Health and Science University, Portland, OR, USA. ${ }^{490}$ Department of Surgery, Duke University, Durham, NC, USA. ${ }^{491}$ Institució Catalana de Recerca i Estudis Avançats (ICREA), Barcelona, Spain. ${ }^{492}$ Institut Català de Paleontologia Miquel Crusafont, Universitat Autònoma de Barcelona, Barcelona, Spain. ${ }^{493}$ University of Glasgow, Glasgow, UK. ${ }^{494}$ Institut $\mathrm{d}^{\prime}$ Investigacions Biomèdiques August Pi i Sunyer (IDIBAPS), Barcelona, Spain. ${ }^{495}$ Division of Oncology, Washington University School of Medicine, St. Louis, MO, USA. ${ }^{496}$ Department of Surgery and Cancer, Imperial College, London, INY, UK. ${ }^{497}$ Applications Department, Oxford Nanopore Technologies, Oxford, UK. ${ }^{498}$ Department of Obstetrics, Gynecology and Reproductive Services, University of California San Francisco, San Francisco, CA, USA. ${ }^{499}$ Department of Biochemistry and Molecular Medicine, University California at Davis, Sacramento, CA, USA. ${ }^{500}$ STTARR Innovation Facility, Princess Margaret Cancer Centre, Toronto, ON, Canada. ${ }^{501}$ Discipline of Surgery, Western Sydney University, Penrith, NSW, Australia. ${ }^{502}$ Yale School of Medicine, Yale University, New Haven, CT, USA. ${ }^{503}$ Department of Genetics, Lineberger Comprehensive Cancer Center, University of North Carolina at Chapel Hill, Chapel Hill, NC, USA. ${ }^{504}$ Departments of Neurology and Neurosurgery, Henry Ford Hospital, Detroit, MI, USA. ${ }^{505}$ Precision Oncology, OHSU Knight Cancer Institute, Oregon Health and Science University, Portland, OR, USA. ${ }^{506}$ Institute of Pathology, University Medical Center Hamburg-Eppendorf, Hamburg, Germany. ${ }^{507}$ Department of Health Sciences, Faculty of Medical Sciences, Kyushu University, Fukuoka, Japan. ${ }^{508}$ Heidelberg Academy of Sciences and Humanities, Heidelberg, Germany. ${ }^{509}$ Department of Clinical Pathology, University of Melbourne, Melbourne, VIC, Australia. ${ }^{510}$ Department of Pathology, Roswell Park Cancer Institute, Buffalo, NY, USA. ${ }^{51}$ Department of Computer Science, University of Helsinki, Helsinki, Finland. ${ }^{512}$ Institute of Biotechnology, University of Helsinki, Helsinki, Finland. ${ }^{513}$ Organismal and Evolutionary Biology Research Programme, University of Helsinki, Helsinki, Finland. ${ }^{514}$ Department of Obstetrics and Gynecology, Division of Gynecologic Oncology, Washington University School of Medicine, St. Louis, MO, USA. ${ }^{515}$ Penrose St. Francis Health Services, Colorado Springs, CO, USA. ${ }^{516}$ Institute of Pathology, Ulm University and University Hospital of Ulm, Ulm, Germany. ${ }^{517}$ National Cancer Center, Tokyo, Japan. ${ }^{518}$ Genome Institute of Singapore, Singapore, Singapore. ${ }^{519} 32$ Program in Computational Biology and Bioinformatics, Yale University, New Haven, CT, USA. ${ }^{520}$ German Cancer Aid, Bonn, Germany. ${ }^{521}$ Programme in Cancer and Stem Cell Biology, Centre for Computational Biology, Duke-NUS Medical School, Singapore, Singapore. ${ }^{522}$ The Chinese University of Hong Kong, Shatin, NT, Hong Kong, China. ${ }^{523}$ Fourth Military Medical University, Shaanxi, China. ${ }^{524}$ The University of Cambridge School of Clinical Medicine, Cambridge, UK. ${ }^{525}$ St. Jude Children's Research Hospital, Memphis, TN, USA. ${ }^{526}$ University Health Network, Princess Margaret Cancer Centre, Toronto, ON, Canada. ${ }^{527}$ Center for Biomolecular Science and Engineering, University of California Santa Cruz, Santa Cruz, CA, USA. ${ }^{528}$ Department of Medicine, University of Chicago, Chicago, IL, USA. ${ }^{529}$ Department of Neurology, Mayo Clinic, Rochester, MN, USA.

${ }^{530}$ Cambridge Oesophagogastric Centre, Cambridge University Hospitals NHS Foundation Trust, Cambridge, UK. ${ }^{531}$ Department of Computer Science, Carleton College, Northfield, MN, USA. ${ }^{532}$ Institute of Cancer Sciences, College of Medical Veterinary and Life Sciences, University of Glasgow, Glasgow, UK. ${ }^{533}$ Department of Epidemiology, University of Alabama at Birmingham, Birmingham, AL, USA. ${ }^{534}$ HudsonAlpha Institute for Biotechnology, Huntsville,
AL, USA. ${ }^{535} \mathrm{O}$ 'Neal Comprehensive Cancer Center, University of Alabama at Birmingham, Birmingham, AL, USA. ${ }^{536}$ Department of Pathology, Keio University School of Medicine, Tokyo, Japan. ${ }^{537}$ Department of Hepatobiliary and Pancreatic Oncology, National Cancer Center Hospital, Tokyo, Japan. ${ }^{538}$ Sage Bionetworks, Seattle, WA, USA. ${ }^{539}$ Lymphoma Genomic Translational Research Laboratory, National Cancer Centre, Singapore, Singapore. ${ }^{540}$ Department of Clinical Pathology, Robert-Bosch-Hospital, Stuttgart, Germany. ${ }^{541}$ Department of Cell and Systems Biology, University of Toronto, Toronto, ON, Canada. ${ }^{542}$ Department of Biosciences and Nutrition, Karolinska Institutet, Stockholm, Sweden. ${ }^{543}$ Center for Liver Cancer, Research Institute and Hospital, National Cancer Center, Gyeonggi, South Korea. ${ }^{54}$ Division of Hematology-Oncology, Samsung Medical Center, Sungkyunkwan University School of Medicine, Seoul, South Korea. ${ }^{545}$ Samsung Advanced Institute for Health Sciences and Technology, Sungkyunkwan University School of Medicine, Seoul, South Korea. ${ }^{546}$ Cheonan Industry-Academic Collaboration Foundation, Sangmyung University, Cheonan, South Korea. ${ }^{547}$ NYU Langone Medical Center, New York, NY, USA. ${ }^{548}$ Department of Hematology and Medical Oncology, Cleveland Clinic, Cleveland, OH, USA. ${ }^{549}$ Department of Radiation Oncology, University of California San Francisco, San Francisco, CA, USA. ${ }^{550}$ Department of Health Sciences Research, Mayo Clinic, Rochester, MN, USA. ${ }^{551}$ Helen F. Graham Cancer Center at Christiana Care Health Systems, Newark, DE, USA. ${ }^{552}$ Heidelberg University Hospital, Heidelberg, Germany. ${ }^{553}$ CSRA Incorporated, Fairfax, VA, USA. ${ }^{554}$ Research Department of Pathology, University College London Cancer Institute, London, UK. ${ }^{555}$ Department of Research Oncology, Guy's Hospital, King's Health Partners AHSC, King's College London School of Medicine, London, UK. ${ }^{556}$ Faculty of Medicine and Health Sciences, Macquarie University, Sydney, NSW, Australia. ${ }^{557}$ University Hospital of Minjoz, INSERM UMR 1098, Besançon, France. ${ }^{558}$ Spanish National Cancer Research Centre, Madrid, Spain. ${ }^{559}$ Center of Digestive Diseases and Liver Transplantation, Fundeni Clinical Institute, Bucharest, Romania. ${ }^{560}$ Cureline, Inc, South San Francisco, CA, USA. ${ }^{561}$ St. Luke's Cancer Centre, Royal Surrey County Hospital NHS Foundation Trust, Guildford, UK. ${ }^{562}$ Cambridge Breast Unit, Addenbrooke's Hospital, Cambridge University Hospital NHS Foundation Trust and NIHR Cambridge Biomedical Research Centre, Cambridge, UK. ${ }^{563}$ East of Scotland Breast Service, Ninewells Hospital, Aberdeen, UK. ${ }^{564}$ Department of Genetics, Microbiology and Statistics, University of Barcelona, IRSJD, IBUB, Barcelona, Spain. ${ }^{565}$ Department of Obstetrics and Gynecology, Medical College of Wisconsin, Milwaukee, WI, USA. ${ }^{56} \mathrm{Hematology}$ and Medical Oncology, Winship Cancer Institute of Emory University, Atlanta, GA, USA. ${ }^{567}$ Department of Computer Science, Princeton University, Princeton, NJ, USA. ${ }^{568}$ Vanderbilt Ingram Cancer Center, Vanderbilt University, Nashville, TN, USA. ${ }^{569}$ Ohio State University College of Medicine and Arthur G. James Comprehensive Cancer Center, Columbus, OH, USA. ${ }^{570}$ Department of Surgery, Yokohama City University Graduate School of Medicine, Kanagawa, Japan. ${ }^{571}$ Division of Chromatin Networks, German Cancer Research Center (DKFZ) and BioQuant, Heidelberg, Germany. ${ }^{572}$ Research Computing Center, University of North Carolina at Chapel Hill, Chapel Hill, NC, USA. ${ }^{573}$ School of Molecular Biosciences and Center for Reproductive Biology, Washington State University, Pullman, WA, USA. ${ }^{574}$ Finsen Laboratory and Biotech Research and Innovation Centre (BRIC), University of Copenhagen, Copenhagen, Denmark. ${ }^{575}$ Department of Laboratory Medicine and Pathobiology, University of Toronto, Toronto, ON, Canada. ${ }^{576}$ Department of Pathology, Human Oncology and Pathogenesis Program, Memorial Sloan Kettering Cancer Center, New York, NY, USA. ${ }^{577}$ University Hospital Giessen, Pediatric Hematology and Oncology, Giessen, Germany. ${ }^{578}$ Oncologie Sénologie, ICM Institut Régional du Cancer, Montpellier, France. ${ }^{579}$ Institute of Clinical Molecular Biology, Christian-Albrechts-University, Kiel, Germany. ${ }^{580}$ Institute of Pathology, University of Wuerzburg, Wuerzburg, Germany. ${ }^{581}$ Department of Urology, North Bristol NHS Trust, Bristol, UK. ${ }^{582}$ SingHealth, Duke-NUS Institute of Precision Medicine, National Heart Centre Singapore, Singapore, Singapore. ${ }^{583}$ Department of Computer Science, University of Toronto, Toronto, ON, Canada. ${ }^{584}$ Bern Center for Precision Medicine, University Hospital of Bern, University of Bern, Bern, Switzerland. ${ }^{585}$ Englander Institute for Precision Medicine, Weill Cornell Medicine and New York Presbyterian Hospital, New York, NY, USA. ${ }^{586}$ Meyer Cancer Center, Weill Cornell Medicine, New York, NY, USA. ${ }^{587}$ Pathology and Laboratory, Weill Cornell Medical College, New York, NY, USA. ${ }^{588}$ Vall d'Hebron Institute of Oncology: VHIO, Barcelona, Spain. ${ }^{589} \mathrm{General}$ and Hepatobiliary-Biliary Surgery, Pancreas Institute, University and Hospital Trust of Verona, Verona, Italy. ${ }^{590}$ National Centre for Biological Sciences, Tata Institute of Fundamental Research, Bangalore, India. ${ }^{591}$ Indiana University, Bloomington, IN, USA. ${ }^{592}$ Department of Pathology, GZA-ZNA Hospitals, Antwerp, Belgium. ${ }^{593}$ Analytical Biological Services, Inc, Wilmington, DE, USA. ${ }^{594}$ Sydney Medical School, University of Sydney, Sydney, NSW, Australia. ${ }^{595} \mathrm{CBio}$ Center, Dana-Farber Cancer Institute, Harvard Medical School, Boston, MA, USA. ${ }^{596}$ Department of Cell Biology, Harvard Medical School, Boston, MA, USA. ${ }^{597}$ Advanced Centre for Treatment Research and Education in Cancer, Tata Memorial Centre, Navi Mumbai, Maharashtra, India. ${ }^{598}$ School of Environmental and Life Sciences, Faculty of Science, The University of Newcastle, Ourimbah, NSW, Australia. ${ }^{599}$ Department of Dermatology, University Hospital of Essen, Essen, Germany. ${ }^{600}$ Bioinformatics and Omics Data Analytics, German Cancer Research Center (DKFZ), Heidelberg, Germany. ${ }^{601}$ Department of Urology, Charité Universitätsmedizin Berlin, Berlin, Germany. ${ }^{602}$ Martini-Clinic, Prostate Cancer Center, University Medical Center Hamburg-Eppendorf, Hamburg, Germany. ${ }^{603}$ Department of General Internal Medicine, University of Kiel, Kiel, Germany. ${ }^{604}$ German Cancer Consortium (DKTK), Partner site Berlin, Berlin, Germany. ${ }^{605}$ Cancer Research Institute, Beth Israel Deaconess Medical Center, Boston, MA, USA. ${ }^{606}$ University of Pittsburgh, Pittsburgh, PA, USA. ${ }^{607}$ Department of Ophthalmology and Ocular Genomics Institute, Massachusetts Eye and Ear, Harvard Medical School, Boston, MA, USA. ${ }^{608}$ Center for Psychiatric Genetics, NorthShore University HealthSystem, Evanston, IL, USA. ${ }^{609}$ Van Andel Research Institute, Grand Rapids, MI, USA. ${ }^{610}$ Laboratory of Molecular 


\section{Article}

Medicine, Human Genome Center, Institute of Medical Science, University of Tokyo, Tokyo Japan. ${ }^{611}$ Japan Agency for Medical Research and Development, Tokyo, Japan. ${ }^{612}$ Korea University, Seoul, South Korea. ${ }^{613}$ Murtha Cancer Center, Walter Reed National Military Medical Center, Bethesda, MD, USA. ${ }^{614}$ Human Genetics, University of Kiel, Kiel, Germany. ${ }^{615}$ Department of Oncologic Pathology, Dana-Farber Cancer Institute, Harvard Medical School, Boston, MA, USA. ${ }^{616}$ Oregon Health and Science University, Portland, OR, USA ${ }^{617}$ Center for RNA Interference and Noncoding RNA, The University of Texas MD Anderson Cancer Center, Houston, TX, USA. ${ }^{618}$ Department of Experimental Therapeutics, The University of Texas MD Anderson Cancer Center, Houston, TX, USA. ${ }^{619}$ Department of Gynecologic Oncology and Reproductive Medicine, The University of Texas MD Anderson Cancer Center, Houston, TX, USA. ${ }^{620}$ University Hospitals Coventry and Warwickshire NHS Trust, Coventry, UK. ${ }^{621}$ Department of Radiation Oncology, Radboud University Nijmegen Medical Centre, Nijmegen, GA, The Netherlands. ${ }^{622}$ Institute for Genomics and Systems Biology, University of Chicago, Chicago, IL, USA. ${ }^{623}$ Clinic for Hematology and Oncology, St.-Antonius-Hospital, Eschweiler, Germany. ${ }^{624}$ Computational and Systems Biology Program, Memorial Sloan Kettering Cancer Center, New York, NY, USA. ${ }^{625}$ University of Iceland, Reykjavik, Iceland. ${ }^{626}$ Division of Computational Genomics and Systems Genetics, German Cancer Research Center (DKFZ), Heidelberg, Germany. ${ }^{627}$ Dundee Cancer Centre, Ninewells Hospital, Dundee, UK. ${ }^{628}$ Department for Internal Medicine III, University of Ulm and University Hospital of Ulm, Ulm, Germany. ${ }^{629}$ Institut Curie, INSERM Unit 830, Paris, France. ${ }^{630}$ Department of Gastroenterology and Hepatology, Yokohama City University Graduate School of Medicine, Kanagawa, Japan. ${ }^{631}$ Department of Laboratory Medicine, Radboud University Nijmegen Medical Centre, Nijmegen, GA, The Netherlands. ${ }^{632}$ Division of Cancer Genome Research, German Cancer Research Center (DKFZ), Heidelberg, Germany. ${ }^{633}$ Department of General Surgery, Singapore General Hospital, Singapore, Singapore. ${ }^{634}$ Cancer Science Institute of Singapore, National University of Singapore, Singapore, Singapore. ${ }^{635}$ Department of Medical and Clinical Genetics, Genome-Scale Biology Research Program, University of Helsinki, Helsinki, Finland. ${ }^{636}$ East Anglian Medical Genetics Service, Cambridge University Hospitals NHS Foundation Trust, Cambridge, UK. ${ }^{637}$ Irving Institute for Cancer Dynamics, Columbia University, New York, NY, USA. ${ }^{638}$ Institute of Molecular and Cell Biology, Singapore, Singapore. ${ }^{639}$ Laboratory of Cancer Epigenome, Division of Medical Science, National Cancer Centre Singapore, Singapore, Singapore. ${ }^{640}$ Universite Lyon, INCa-Synergie, Centre Léon Bérard, Lyon, France. ${ }^{641}$ Department of Urology, Mayo Clinic, Rochester, MN, USA. ${ }^{642}$ Royal National Orthopaedic Hospital - Stanmore, Stanmore, Middlesex, UK. ${ }^{643}$ Department of Biochemistry, Genetics and Immunology, University of Vigo Vigo, Spain. ${ }^{644}$ Giovanni Paolo II / I.R.C.C.S. Cancer Institute, Bari, BA, Italy. ${ }^{645}$ Neuroblastoma Genomics, German Cancer Research Center (DKFZ), Heidelberg, Germany. ${ }^{646}$ Fondazione Policlinico Universitario Gemelli IRCCS, Rome, Italy, Rome, Italy. ${ }^{647}$ University of Verona, Verona, Italy. ${ }^{648}$ Centre National de Génotypage, CEA - Institute de Génomique, Evry, France. ${ }^{649}$ CAPHRI Research School, Maastricht University, Maastricht, ER, The Netherlands. ${ }^{650}$ Department of Biopathology, Centre Léon Bérard, Lyon, France. ${ }^{651}$ Université Claude Bernard Lyon 1, Villeurbanne, France. ${ }^{652}$ Core Research for Evolutional Science and Technology (CREST), JST, Tokyo, Japan. ${ }^{653}$ Department of Biological Sciences, Laboratory for Medical Science Mathematics, Graduate School of Science, University of Tokyo, Yokohama, Japan. ${ }^{654}$ Department of Medical Science Mathematics, Medical Research Institute, Tokyo Medical and Dental University (TMDU), Tokyo, Japan. ${ }^{655}$ Cancer Ageing and Somatic Mutation Programme, Wellcome Sanger Institute, Hinxton, UK. ${ }^{656}$ University Hospitals Birmingham NHS Foundation Trust, Birmingham, UK. ${ }^{657}$ Centre for Cancer Research and Cell Biology, Queen's University, Belfast, UK. ${ }^{658}$ Breast Medical Oncology, The University of Texas MD Anderson Cancer Center, Houston, TX, USA. ${ }^{659}$ Department of Surgery, Johns Hopkins University School of Medicine, Baltimore, MD, USA. ${ }^{660}$ Department of Oncology-Pathology, Science for Life Laboratory, Karolinska Institute, Stockholm, Sweden. ${ }^{661}$ School of Cancer Sciences, Faculty of Medicine, University of Southampton, Southampton, UK. ${ }^{662}$ Department of Gene Technology, Tallinn University of Technology, Tallinn, Estonia. ${ }^{663}$ Genetics and Genome Biology Program, SickKids Research Institute, The Hospital for Sick Children, Toronto, ON, Canada. ${ }^{664}$ Departments of Neurosurgery and Hematology and Medical Oncology, Winship Cancer Institute and School of Medicine, Emory University, Atlanta, GA, USA. ${ }^{665}$ Department of Clinical and Molecular Medicine, Faculty of Medicine and Health Sciences, Norwegian University of Science and Technology, Trondheim, Norway. ${ }^{666}$ Argmix Consulting, North Vancouver, BC, Canada. ${ }^{667}$ Department of Information Technology, Ghent University,
Interuniversitair Micro-Electronica Centrum (IMEC), Ghent, Belgium. ${ }^{668}$ Nuffield Department of Surgical Sciences, John Radcliffe Hospital, University of Oxford, Oxford, UK. ${ }^{669}$ Institute of Mathematics and Computer Science, University of Latvia, Riga, LV, Latvia. ${ }^{670}$ Discipline of Pathology, Sydney Medical School, University of Sydney, Sydney, NSW, Australia. ${ }^{671}$ Department of Applied Mathematics and Theoretical Physics, Centre for Mathematical Sciences, University of Cambridge, Cambridge, UK. ${ }^{672}$ Department of Epidemiology and Biostatistics, Memorial Sloan Kettering Cancer Center, New York, NY, USA. ${ }^{673}$ Department of Statistics, Columbia University, New York, NY, USA. ${ }^{674}$ Department of Immunology, Genetics and Pathology, Science for Life Laboratory, Uppsala University, Uppsala, Sweden. ${ }^{675}$ School of Electronic and Information Engineering, Xi'an Jiaotong University, Xi'an, China. ${ }^{676}$ Department of Histopathology, Cambridge University Hospitals NHS Foundation Trust, Cambridge, UK. ${ }^{677}$ Oxford NIHR Biomedical Research Centre, University of Oxford, Oxford, UK. ${ }^{678}$ Georgia Regents University Cancer Center, Augusta, GA, USA. ${ }^{679}$ Wythenshawe Hospital, Manchester, UK. ${ }^{680}$ Department of Genetics, Washington University School of Medicine, St.Louis, MO, USA. ${ }^{681}$ Department of Biological Oceanography, Leibniz Institute of Baltic Sea Research, Rostock, Germany. ${ }^{682}$ Wellcome Centre for Human Genetics, University of Oxford, Oxford, UK. ${ }^{683}$ Department of Molecular and Human Genetics, Baylor College of Medicine, Houston, TX, USA. ${ }^{684}$ Thoracic Oncology Laboratory, Mayo Clinic, Rochester, MN, USA. ${ }^{685}$ Institute for Genomic Medicine, Nationwide Children's Hospital, Columbus, OH, USA. ${ }^{686}$ Department of Obstetrics and Gynecology, Division of Gynecologic Oncology, Mayo Clinic, Rochester, MN, USA. ${ }^{687}$ International Institute for Molecular Oncology, Poznań, Poland. ${ }^{688}$ Poznan University of Medical Sciences, Poznań, Poland. ${ }^{689}$ Genomics and Proteomics Core Facility High Throughput Sequencing Unit, German Cancer Research Center (DKFZ), Heidelberg, Germany. ${ }^{690}$ NCCS-VARI Translational Research Laboratory, National Cancer Centre Singapore, Singapore, Singapore. ${ }^{691}$ Edison Family Center for Genome Sciences and Systems Biology, Washington University, St. Louis, MO, USA. ${ }^{692} \mathrm{MRC}-$ University of Glasgow Centre for Virus Research, Glasgow, UK. ${ }^{693}$ Department of Medical Informatics and Clinical Epidemiology, Division of Bioinformatics and Computational Biology, OHSU Knight Cancer Institute, Oregon Health and Science University, Portland, OR, USA. ${ }^{694}$ School of Electronic Information and Communications, Huazhong University of Science and Technology, Wuhan, China. ${ }^{695}$ Department of Applied Mathematics and Statistics, Johns Hopkins University, Baltimore, MD, USA. ${ }^{696}$ Department of Cancer Genome Informatics, Graduate School of Medicine, Osaka University, Osaka, Japan. ${ }^{697}$ Institute of Computer Science, Heidelberg University, Heidelberg, Germany ${ }^{698}$ School of Mathematics and Statistics, University of Sydney, Sydney, NSW, Australia. ${ }^{69}$ Ben May Department for Cancer Research, University of Chicago, Chicago, IL, USA. ${ }^{700}$ Department of Human Genetics, University of Chicago, Chicago, IL, USA. ${ }^{701}$ Tri-Institutional PhD Program in Computational Biology and Medicine, Weill Cornell Medicine, New York, NY, USA. ${ }^{702}$ The First Affiliated Hospital, Xi'an Jiaotong University, Xi'an, China. ${ }^{703}$ Department of Medicine and Therapeutics, The Chinese University of Hong Kong, Shatin, NT, Hong Kong, China. ${ }^{704}$ Department of Biostatistics, The University of Texas MD Anderson Cancer Center, Houston, TX, USA. ${ }^{705}$ Duke-NUS Medical School, Singapore, Singapore. ${ }^{706}$ Department of Surgery, Ruijin Hospital, Shanghai Jiaotong University School of Medicine, Shanghai, China. ${ }^{707}$ School of Computing Science, University of Glasgow, Glasgow, UK. ${ }^{708}$ Division of Orthopaedic Surgery, Oslo University Hospital, Oslo, Norway. ${ }^{709}$ Eastern Clinical School, Monash University, Melbourne, VIC, Australia. ${ }^{710}$ Epworth HealthCare, Richmond, VIC, Australia. ${ }^{71}$ Department of Biostatistics and Computational Biology, Dana-Farber Cancer Institute and Harvard Medical School, Boston, MA, USA. ${ }^{712}$ Department of Biomedical Informatics, College of Medicine, The Ohio State University, Columbus, OH, USA. ${ }^{713}$ The Ohio State University Comprehensive Cancer Center (OSUCCC - James), Columbus, $\mathrm{OH}$, USA. ${ }^{714}$ The University of Texas School of Biomedical Informatics (SBMI) at Houston, Houston, TX, USA. ${ }^{715}$ Department of Biostatistics, University of North Carolina at Chapel Hill, Chapel Hill, NC, USA. ${ }^{716}$ Department of Biochemistry and Molecular Genetics, Feinberg School of Medicine, Northwestern University, Chicago, IL, USA. ${ }^{717}$ Faculty of Medicine and Health, University of Sydney, Sydney, NSW, Australia. ${ }^{718}$ Department of Pathology, Erasmus Medical Center Rotterdam, Rotterdam, GD, The Netherlands. ${ }^{719}$ Division of Molecular Carcinogenesis, The Netherlands Cancer Institute, Amsterdam, CX, The Netherlands. ${ }^{720}$ Institute of Molecular Life Sciences and Swiss Institute of Bioinformatics, University of Zurich, Zurich, Switzerland. ${ }^{721}$ These authors jointly supervised this work: Peter J. Campbell, Gad Getz, Jan O. Korbel, Joshua M. Stuart, Lincoln D. Stein. *e-mail: pc8@sanger.ac.uk; gadgetz@broadinstitute.org; korbel@embl.de; jstuart@ucsc.edu; lincoln.stein@gmail.com 


\section{Methods}

\section{Samples}

We compiled an inventory of matched tumour-normal whole-cancer genomes in the ICGC Data Coordinating Centre. Most samples came from treatment-naive, primary cancers, although a small number of donors had multiple samples of primary, metastatic and/or recurrent tumours. Our inclusion criteria were: (1) matched tumour and normal specimen pair; (2) a minimal set of clinical fields; and (3) characterization of tumour and normal whole genomes using Illumina HiSeq paired-end sequencing reads.

We collected genome data from 2,834 donors, representing all ICGC and TCGA donors that met these criteria at the time of the final data freeze in autumn 2014 (Extended Data Table 1). After quality assurance (Supplementary Methods 2.5), data from 176 donors were excluded as unusable, 75 had minor issues that could affect some analyses (grey-listed donors) and 2,583 had data of optimal quality (white-listed donors) (Supplementary Table 1). Across the 2,658 white- and greylisted donors, whole-genome sequences were available from 2,605 primary tumours and 173 metastases or local recurrences. Matching normal samples were obtained from blood (2,064 donors), tissue adjacent to the primary tumour ( 87 donors) or from distant sites (507 donors). Whole-genome sequencing data were available for tumour and normal DNA for the entire cohort. The mean read coverage was $39 \times$ for normal samples, whereas tumours had a bimodal coverage distribution with modes at $38 \times$ and $60 \times$ (Supplementary Fig. 1 ). The majority of specimens (65.3\%) were sequenced using 101-bp pairedend reads. An additional $28 \%$ were sequenced with 100 -bp paired-end reads. Of the remaining specimens, $4.7 \%$ were sequenced with read lengths longer than $101 \mathrm{bp}$, and $1.9 \%$ with read lengths shorter than $100 \mathrm{bp}$. The distribution of read lengths by tumour cohort is shown in Supplementary Fig. 11. Median read length for whole-genome sequencing paired-end reads was $101 \mathrm{bp}$ ( mean $=106.2$, s.d. $=16.7$; minimum-maximum $=50-151$ ). RNA-sequencing data were collected and re-analysed centrally for 1,222 donors, including 1,178 primary tumours, 67 metastases or local recurrences and 153 matched normal tissue samples adjacent to the primary tumour.

Demographically, the cohort included 1,469 men (55\%) and 1,189 women ( $45 \%$ ), with a mean age of 56 years (range, $1-90$ years) (Supplementary Table 1). Using population ancestry-differentiated single nucleotide polymorphisms, the ancestry distribution was heavily weighted towards donors of European descent ( $77 \%$ of total) followed by East Asians (16\%), as expected for large contributions from European, North American and Australian projects (Supplementary Table 1).

We consolidated histopathology descriptions of the tumour samples, using the ICD-0-3 tumour site controlled vocabulary ${ }^{89}$. Overall, the PCAWG dataset comprises 38 distinct tumour types (Extended Data Table 1 and Supplementary Table 1). Although the most common tumour types are included in the dataset, their distribution does not match the relative population incidences, largely owing to differences among contributing ICGC/TCGA groups in the numbers of sequenced samples.

\section{Uniform processing and somatic variant calling}

To generate a consistent set of somatic mutation calls that could be used for cross-tumour analyses, we analysed all 6,835 samples using a uniform set of algorithms for alignment, variant calling and quality control (Extended Data Fig. 1, Supplementary Fig. 2, Supplementary Table 3 and Supplementary Methods 2). We used the BWA-MEM algorithm ${ }^{90}$ to align each tumour and normal sample to human reference build hs37d5 (as used in the 1000 Genomes Project ${ }^{91}$ ). Somatic mutations were identified in the aligned data using three established pipelines, which were run independently on each tumour-normal pair. Each of the three pipelines-labelled 'Sanger' ${ }^{\prime 22-95}$,'EMBL/DKFZ'96,97 and 'Broad' ${ }^{\text {'98-101 }}$ after the computational biology groups that created or assembled them-consisted of multiple software packages for calling somatic SNVs, small indels, CNAs and somatic SVs (with intrachromosomal SVs defined as those $>100 \mathrm{bp}$ ). Two additional variant algorithms ${ }^{102,103}$ were included to further improve accuracy across a broad range of clonal and subclonal mutations. We tested different merging strategies using validation data, and choses the optimal method for each variant type to generate a final consensus set of mutation calls (Supplementary Methods S2.4).

Somatic retrotransposition events, including Alu and LINE-1 insertions $^{72}$, L1-mediated transductions ${ }^{73}$ and pseudogene formation ${ }^{104}$, were called using a dedicated pipeline ${ }^{73}$. We removed these retrotransposition events from the somatic SV call-set. Mitochondrial DNA mutations were called using a published algorithm ${ }^{105}$. RNA-sequencing data were uniformly processed to quantify normalized gene-level expression, splicing variation and allele-specific expression, and to identify fusion transcripts, alternative promoter usage and sites of RNA editing ${ }^{8}$.

Integration, phasing and validation of germline variant call-sets Calls of common ( $\geq 1 \%$ frequency in PCAWG) and rare $(<1 \%)$ germline variants including single-nucleotide polymorphisms, indels, SVs and mobile-element insertions (MEIs) were generated using a populationscale genetic polymorphism-detection approach ${ }^{11,106}$. The uniform germline data-processing workflow comprised variant identification using six different variant-calling algorithms $\mathrm{s}^{96,107,108}$ and was orchestrated using the Butler workflow system ${ }^{109}$.

We performed call-set benchmarking, merging, variant genotyping and statistical haplotype-block phasing ${ }^{91}$ (Supplementary Methods 3.4). Using this strategy, we identified 80.1 million germline single-nucleotide polymorphisms, 5.9 million germline indels, 1.8 million multi-allelic short ( $<50 \mathrm{bp}$ ) germline variants, as well as germline SVs $\geq 50$ bp in size including 29,492 biallelic deletions and 27,254 MEIs (Supplementary Table 2). We statistically phased this germline variant set using haplotypes from the 1000 Genomes Project ${ }^{91}$ as a reference panel, yielding an N50-phased block length of $265 \mathrm{~kb}$ based on haploid chromosomes from donor-matched tumour genomes. Precision estimates for germline SNVs and indels were $>\mathbf{9 9 \%}$ for the phased merged call-set, and sensitivity estimates ranged from $92 \%$ to $98 \%$.

\section{Core alignment and variant calling by cloud computing}

The requirement to uniformly realign and call variants on nearly 5,800 whole genomes (tumour plus normal) presented considerable computational challenges, and raised ethical issues owing to the use of data from different jurisdictions (Extended Data Table 2). To process the data, we adopted a cloud-computing architecture ${ }^{26}$ in which the alignment and variant calling was spread across 13 data centres on 3 continents, representing a mixture of commercial, infrastructure-asa-service, academic cloud compute and traditional academic highperformance computer clusters (Supplementary Table 3). Together, the effort used 10 million CPU-core hours.

To generate reproducible variant calling across the 13 data centres, we built the core pipelines into Docker containers ${ }^{28}$, in which the workflow description, required code and all associated dependencies were packaged together in stand-alone packages. These heavily tested, extensively validated workflows are available for download (Box 1).

\section{Validation, benchmarking and merging of somatic variant calls} To evaluate the performance of each of the mutation-calling pipelines and determine an integration strategy, we performed a large-scale deep-sequencing validation experiment (Supplementary Notes 1 ). We selected a pilot set of 63 representative tumour-normal pairs, on which we ran the 3 core pipelines, together with a set of 10 additional somatic variant-calling pipelines contributed by members of the PCAWG SNV Calling Methods Working Group. Sufficient DNA remained for 50 of the 63 cases for validation, which was performed by hybridization of tumour and matched normal DNA to a custom RNA bait set, followed 
by deep sequencing, as previously described ${ }^{29}$. Although performed using the same sequencing chemistry as the original whole-genome sequencing analyses, the considerably greater depth achieved in the validation experiment enabled accurate assessment of sensitivity and precision of variant calls. Variant calls in repeat-masked regions were not tested, owing to the challenge of designing reliable validation probes in these areas.

The 3 core pipelines had individual estimates of sensitivity of $80-90 \%$ to detect a true somatic SNV called by any of the 13 pipelines; with $>95 \%$ of SNV calls made by each of the core pipelines being genuine somatic variants (Fig. 1a). For indels-a more-challenging class of variants to identify in short-read sequencing data-the 3 core algorithms had individual sensitivity estimates in the range of $40-50 \%$, with precision 70-95\% (Fig. 1b). Validation of SV calls is inherently more difficult, as methods based on PCR or hybridization to RNA baits often fail to isolate DNA that spans the breakpoint. To assess the accuracy of SV calls, we therefore used the property that an SV must either generate a copy-number change or be balanced, whereas artefactual calls will not respect this property. For individual SV-calling algorithms, we estimated precision to be in the range of $80-95 \%$ for samples in the 63-sample pilot dataset.

Next, we examined multiple methods for merging calls made by several algorithms into a single definitive call-set to be used for downstream analysis. The final consensus calls for SNVs were based on a simple approach that required two or more methods to agree on a call. For indels, because methods were less concordant, we used stacked logistic regression ${ }^{110,111}$ to integrate the calls. The merged SV set includes all calls made by two or more of the four primary SV-calling algorithms ${ }^{96,100,112,113}$. Consensus CNA calls were obtained by joining the outputs of six individual CNA-calling algorithms with SV consensus breakpoints to obtain base-pair resolution CNAs (Supplementary Methods 2.4.3). Consensus purity and ploidy were derived, and a multitier system was developed for consensus copy-number calls (Supplementary Methods 2.4.3, and described in detail elsewhere ${ }^{7}$ ).

Overall, the sensitivity and precision of the consensus somatic variant calls were $95 \%$ (90\% confidence interval, 88-98\%) and $95 \%(90 \%$ confidence interval, 71-99\%), respectively, for SNVs (Extended Data Fig. 2). For somatic indels, sensitivity and precision were $60 \%$ (90\% confidence interval, 34-72\%) and $91 \%$ (90\% confidence interval, 73-96\%), respectively. Regarding SVs, we estimate the sensitivity of the merging algorithm to be $90 \%$ for true calls generated by any one calling pipeline; precision was estimated to be $97.5 \%$. That is, $97.5 \%$ of SVs in the merged SV call-set had an associated copy-number change or balanced partner rearrangement. The improvement in calling accuracy from combining different pipelines was most noticeable in variants that had low variant allele fractions, which are likely to originate from subclonal populations of the tumour (Fig. 1c, d). There remains much work to be done to improve indel calling software; we still lack sensitivity for calling even fully clonal complex indels from short-read sequencing data.

\section{Reporting summary}

Further information on research design is available in the Nature Research Reporting Summary linked to this paper.

\section{Data availability}

The PCAWG-generated alignments, somatic variant calls, annotations and derived datasets are available for general research use for browsing and download at http://dcc.icgc.org/pcawg/ (Box 1 and Supplementary Table 4). In accordance with the data access policies of the ICGC and TCGA projects, most molecular, clinical and specimen data are in an open tier which does not require access approval. To access potentially identifying information, such as germline alleles and underlying read data, researchers will need to apply to the TCGA Data Access Committee (DAC) via dbGaP (https://dbgap.ncbi.nlm.nih.gov/aa/wga. cgi?page=login) for access to the TCGA portion of the dataset, and to the ICGC Data Access Compliance Office (DACO; http://icgc.org/daco) for the ICGC portion. In addition, to access somatic single nucleotide variants derived from TCGA donors, researchers will also need to obtain dbGaP authorization.

Beyond the core sequence data and variant call-sets, the analyses in this paper used a number of datasets that were derived from the variant calls (Supplementary Table 4). The individual datasets are available at Synapse (https://www.synapse.org/), and are denoted with synXXXXX accession numbers; all these datasets are also mirrored at https://dcc. icgc.org, with full links, filenames, accession numbers and descriptions detailed in Supplementary Table 4. The datasets encompass: clinical data from each patient including demographics, tumour stage and vital status (syn10389158); harmonized tumour histopathology annotations using a standardised hierarchical ontology (syn1038916); inferred purity and ploidy values for each tumour sample (syn8272483); driver mutations for each patient from their cancer genome spanning all classes of variant, and coding versus non-coding drivers (syn11639581); mutational signatures inferred from PCAWG donors (syn11804065), including APOBEC mutagenesis (syn7437313); and transcriptional data from RNA sequencing, including gene expression levels (syn5553985, syn5553991, syn8105922) and gene fusions (syn10003873, syn7221157).

\section{Code availability}

Computational pipelines for calling somatic mutations are available to the public at https://dockstore.org/organizations/PCAWG/collections/ PCAWG. A range of data-visualization and -exploration tools are also available for the PCAWG data (Box 1).

89. NCI SEER. ICD-O-3 Coding Materials (2018).

90. Li, H. \& Durbin, R. Fast and accurate long-read alignment with Burrows-Wheeler transform. Bioinformatics 26, 589-595 (2010).

91. 1000 Genomes Project Consortium. A global reference for human genetic variation. Nature 526, 68-74 (2015)

92. Raine, K. M. et al. ascatNgs: identifying somatically acquired copy-number alterations from whole-genome sequencing data. Curr. Protoc. Bioinformatics 56, 15.9.1-15.9.17 (2016).

93. Jones, D. et al. cgpCaVEManWrapper: simple execution of CaVEMan in order to detect somatic single nucleotide variants in NGS data. Curr. Protoc. Bioinformatics 56, 15.10.1-15.10.18 (2016).

94. Raine, K. M. et al. cgpPindel: identifying somatically acquired insertion and deletion events from paired end sequencing. Curr. Protoc. Bioinformatics 52, 15.7.1-15.7.12 (2015).

95. Ye, K., Schulz, M. H., Long, Q., Apweiler, R. \& Ning, Z. Pindel: a pattern growth approach to detect break points of large deletions and medium sized insertions from paired-end short reads. Bioinformatics 25, 2865-2871 (2009).

96. Rausch, T. et al. DELLY: structural variant discovery by integrated paired-end and splitread analysis. Bioinformatics 28, i333-i339 (2012).

97. Rimmer, A. et al. Integrating mapping-, assembly- and haplotype-based approaches for calling variants in clinical sequencing applications. Nat. Genet. 46, 912-918 (2014).

98. Cibulskis, K. et al. Sensitive detection of somatic point mutations in impure and heterogeneous cancer samples. Nat. Biotechnol. 31, 213-219 (2013).

99. Carter, S. L. et al. Absolute quantification of somatic DNA alterations in human cancer. Nat. Biotechnol. 30, 413-421 (2012)

100. Drier, Y. et al. Somatic rearrangements across cancer reveal classes of samples with distinct patterns of DNA breakage and rearrangement-induced hypermutability. Genome Res. 23, 228-235 (2013).

101. Ramos, A. H. et al. Oncotator: cancer variant annotation tool. Hum. Mutat. 36, E2423-E2429 (2015).

102. Moncunill, V. et al. Comprehensive characterization of complex structural variations in cancer by directly comparing genome sequence reads. Nat. Biotechnol. 32, 1106-1112 (2014).

103. Fan, Y. et al. MuSE: accounting for tumor heterogeneity using a sample-specific error model improves sensitivity and specificity in mutation calling from sequencing data. Genome Biol. 17, 178 (2016).

104. Cooke, S. L. et al. Processed pseudogenes acquired somatically during cancer development. Nat. Commun. 5, 3644 (2014).

105. Ju, Y. S. et al. Origins and functional consequences of somatic mitochondrial DNA mutations in human cancer. eLife 3, e02935 (2014).

106. Sudmant, P. H. et al. An integrated map of structural variation in 2,504 human genomes. Nature 526, 75-81 (2015).

107. Garrison, E. \& Marth, G. Haplotype-based variant detection from short-read sequencing. Preprint at https://arxiv.org/abs/1207.3907 (2012).

108. DePristo, M. A. et al. A framework for variation discovery and genotyping using nextgeneration DNA sequencing data. Nat. Genet. 43, 491-498 (2011). 
109. Yakneen, S., Waszak, S. M., Gertz, M. \& Korbel, J. O. \& PCAWG Consortium. Butler enables rapid cloud-based analysis of thousands of human genomes. Nat. Biotechnol. https://doi. org/10.1038/s41587-019-0360-3 (2020).

110. Kim, S. Y., Jacob, L. \& Speed, T. P. Combining calls from multiple somatic mutationcallers. BMC Bioinformatics 15, 154 (2014).

111. Breiman, L. Stacked regressions. Mach. Learn. 24, 49-64 (1996).

112. Campbell, P. J. et al. Identification of somatically acquired rearrangements in cancer using genome-wide massively parallel paired-end sequencing. Nat. Genet. 40, 722-729 (2008).

113. Wala, J. A. et al. SvABA: genome-wide detection of structural variants and indels by local assembly. Genome Res. 28, 581-591 (2018).

Acknowledgements We thank research participants who donated samples and data, the physicians and clinical staff who contributed to sample annotation and collection, and the numerous funding agencies that contributed to the collection and analysis of this dataset.

Author contributions Writing committee leads: Peter J. Campbell, Gad Getz, Jan O. Korbel, Joshua M. Stuart, Jennifer L. Jennings, Lincoln D. Stein. Head of project management: Jennife L. Jennings. Sample collection: major contributions from Marc D. Perry, Hardeep K. NahalBose; led by B. F. Francis Ouellette. Histopathology harmonization: major contribution from Constance H. Li; further contributions from Esther Rheinbay, G. Petur Nielsen, Dennis C. Sgroi, Chin-Lee Wu, William C. Faquin, Vikram Deshpande, Paul C. Boutros, Alexander J. Lazar, Katherine A. Hoadley; led by Lincoln D. Stein, David N. Louis. Uniform processing, somatic, germline variant calling: major contribution from L. Jonathan Dursi; further contributions from Christina K. Yung, Matthew H. Bailey, Gordon Saksena, Keiran M. Raine, Ivo Buchhalter, Kortine Kleinheinz, Matthias Schlesner, Junjun Zhang, Wenyi Wang, David A. Wheeler; led by Li Ding, Jared T. Simpson. Core alignment, variant calling by cloud computing: major contributions from Christina K. Yung, Brian D. O'Connor, Sergei Yakneen, Junjun Zhang. further contributions from Kyle Ellrott, Kortine Kleinheinz, Naoki Miyoshi, Keiran M. Raine, Adam P. Butler, Romina Royo, Gordon Saksena, Matthias Schlesner, Solomon I. Shorser, Miguel Vazquez. Integration, phasing validation of germline variant callsets: major contributions from Tobias Rausch, Grace Tiao, Sebastian M. Waszak, Bernardo Rodriguez-Martin, Suyash Shringarpure, Dai-Ying Wu; further contributions from Sergei Yakneen, German M. Demidov, Olivier Delaneau, Shuto Hayashi, Seiya Imoto, Nina Habermann, Ayellet V. Segre, Erik Garrison, Andy Cafferkey, Eva G. Alvarez, José María Heredia-Genestar, Francesc Muyas, Oliver Drechsel, Alicia L. Bruzos, Javie Temes, Jorge Zamora, L. Jonathan Dursi, Adrian Baez-Ortega, Hyung-Lae Kim, Matthew H. Bailey, R. Jay Mashl, Kai Ye, Ivo Buchhalter, Anthony DiBiase, Kuan-lin Huang, Ivica Letunic, Michael D. McLellan, Steven J. Newhouse, Matthias Schlesner, Tal Shmaya, Sushant Kumar, David C. Wedge, Mark H. Wright, Venkata D. Yellapantula, Mark Gerstein, Ekta Khurana, Tomas Marques-Bonet, Arcadi Navarro, Carlos D. Bustamante, Jared T. Simpson, Li Ding, Reine Siebert Hidewaki Nakagawa, Douglas F. Easton; led by Stephan Ossowski, Jose M. C. Tubio, Gad Getz, Francisco M. De La Vega, Xavier Estivill, Jan O. Korbel. Validation, benchmarking, merging of somatic variant calls: major contribution from L. Jonathan Dursi; further contributions from David A. Wheeler, Christina K. Yung; led by Li Ding, Jared T. Simpson. Data and code availability: major contribution from Junjun Zhang; further contributions from Christina K. Yung, Sergei Yakneen, Denis Yuen, George L. Mihaiescu, Larsson Omberg; led by Vincent Ferretti. Pan-cancer burden of somatic mutations: major contribution from Junjun Zhang; led by Peter J. Campbell. Panorama of driver mutations in human cancer: led by Radhakrishnan Sabarinathan, Oriol Pich, Abel Gonzalez-Perez. PCAWG tumours with no apparent driver mutations: major contribution from Esther Rheinbay; further contributions from Amaro Taylor-Weiner, Radhakrishnan Sabarinathan; led by Peter J. Campbell, Gad Getz. Patterns, oncogenicity of kataegis, chromoplexy: major contributions from Matthew W. Fittall, Jonas Demeulemeester, Maxime Tarabichi; further contributions from Nicola D. Roberts, Pete J. Campbell, Jan O. Korbel; led by Peter Van Loo. Patterns, oncogenicity of chromothripsis: major contributions from Maxime Tarabichi, Jonas Demeulemeester, Matthew W. Fittall. further contributions from Isidro Cortes-Ciriano, Lara Urban, Peter J. Park, Peter J. Campbell, Jan O. Korbel; led by Peter Van Loo. Timing-clustered mutational processes during tumour evolution: major contributions from Jonas Demeulemeester, Maxime Tarabichi, Matthew W. Fittall; further contributions from Jan O. Korbel, Peter J. Campbell; led by Peter Van Loo. Germline effects on somatic mutation: major contributions from Sebastian M. Waszak, Bin Zhu, Bernardo Rodriguez-Martin, Esa Pitkanen, Tobias Rausch; further contributions from Yilong Li, Natalie Saini, Leszek J. Klimczak, Joachim Weischenfeldt, Nikos Sidiropoulos, Ludmil B. Alexandrov, Francesc Muyas, Raquel Rabionet, Georgia Escaramis, Adrian Baez-Ortega, Mattia Bosio, Aliaksei Z. Holik, Hana Susak, Eva G. Alvarez, Alicia L. Bruzos, Javier Temes, Aparna Prasad, Nina Habermann, Serap Erkek, Lara Urban, Claudia Calabrese, Benjamin Raeder, Eoghan Harrington, Simon Mayes, Daniel Turner, Sissel Juul, Steven A. Roberts, Lei Song, Roelof Koster, Lisa Mirabello, Xing Hua, Tomas J. Tanskanen, Marta Tojo, David C. Wedge, Jorge Zamora, Jieming Chen, Lauri A. Aaltonen, Gunnar Ratsch, Roland F. Schwarz, Atul J. Butte, Alvis Brazma, Peter J. Campbell, Stephen J. Chanock, Nilanjan Chatterjee, Oliver Stegle, Olivier Harismendy; led by G. Steven Bova, Dmitry A. Gordenin, Jose M. C. Tubio, Douglas F. Easton, Xavier Estivill, Jan O. Korbel. Replicative immortality: major contribution from David Haan; further contributions from Lina Sieverling, Lars Feuerbach; led by Lincoln D. Stein, Joshua M. Stuart. Ethical considerations of genomic cloud computing: led by Don Chalmers, Yann Joly, Bartha Knoppers, Fruzsina Molnar-Gabor, Jan O. Korbel, Mark Phillips, Adrian Thorogood, David Townend. Online resources for data access, visualization, exploration and analysis: majo contributions from Mary Goldman, Junjun Zhang, Nuno A. Fonseca; further contributions from Qian Xiang, Brian Craft, Elena Pineiro-Yanez, Alfonso Munoz, Robert Petryszak, Anja Fullgrabe Fatima Al-Shahrour, Maria Keays, David Haussler, John Weinstein, Wolfgang Huber, Alfonso Valencia, Irene Papatheodorou, Jingchun Zhu; led by Brian D. O'Connor, Lincoln D. Stein, Alvis Brazma, Vincent Ferretti, Miguel Vazquez. The 63-sample pilot-analysis validation process: major contribution from L. Jonathan Dursi; further contributions from Christina K. Yung Matthew H. Bailey, Gordon Saksena, Keiran M. Raine, Ivo Buchhalter, Kortine Kleinheinz Matthias Schlesner, Yu Fan, David Torrents, Matthias Bieg, Paul C. Boutros, Ken Chen, Zechen Chong, Kristian Cibulskis, Oliver Drechsel, Roland Eils, Robert S. Fulton, Josep Gelpi, Mark Gerstein, Santiago Gonzalez, Gad Getz, Ivo G. Gut, Faraz Hach, Michael Heinold, Taobo Hu, Vincent Huang, Barbara Hutter, Hyung-Lae Kim, Natalie Jager, Jongsun Jung, Sushant Kumar,
Yogesh Kumar, Christopher Lalansingh, Ignaty Leshchiner, Ivica Letunic, Dimitri Livitz, Eric Z. Ma, Yosef E. Maruvka, R. Jay Mashl, Michael D. McLellan, Ana Milovanovic, Morten Muhlig Nielsen, Brian D. O'Connor, Stephan Ossowski, Nagarajan Paramasivam, Jakob Skou Pedersen, Marc D. Perry, Montserrat Puiggros, Romina Royo, Esther Rheinbay, S. Cenk Sahinalp, Iman Sarrafi, Chip Stewart, Miranda D. Stobbe, Grace Tiao, Jeremiah A. Wala, Jiayin Wang, Weny Wang, Sebastian M. Waszak, Joachim Weischenfeldt, Michael Wendl, Johannes Werner, Zhenggang Wu, Hong Xue, Sergei Yakneen, Takafumi N. Yamaguchi, Kai Ye, Venkata Yellapantula, Junjun Zhang, David A. Wheeler; led by Li Ding, Jared T. Simpson. Processing of validation data: major contributions from Christina K. Yung, Brian D. O'Connor, Sergei Yakneen, Junjun Zhang; further contributions from Kyle Ellrott, Kortine Kleinheinz, Naoki Miyoshi, Keiran M. Raine, Romina Royo, Gordon Saksena, Matthias Schlesner, Solomon I. Shorser, Miguel Vazquez, Joachim Weischenfeldt, Denis Yuen, Adam P. Butler, Brandi N. Davis-Dusenbery, Roland Eils, Vincent Ferretti, Robert L. Grossman, Olivier Harismendy, Youngwook Kim, Hidewaki Nakagawa, Steven J. Newhouse, David Torrents; led by Lincoln D. Stein. Wholegenome sequencing somatic variant calling: major contribution from Junjun Zhang; further contributions from Christina K. Yung, Solomon I. Shorser. Whole-genome alignment: Keiran M. Raine, Junjun Zhang, Brian D. O'Connor. DKFZ pipeline: Kortine Kleinheinz, Tobias Rausch, Jan O. Korbel, Ivo Buchhalter, Michael C. Heinold, Barbara Hutter, Natalie Jager, Nagarajan Paramasivam, Matthias Schlesner. EMBL pipeline: Joachim Weischenfeldt. Sanger pipeline: Keiran M. Raine, Jonathan Hinton, David R. Jones, Andrew Menzies, Lucy Stebbings, Adam P. Butler. Broad pipeline: Gordon Saksena, Dimitri Livitz, Esther Rheinbay, Julian M. Hess, Ignaty eshchiner, Chip Stewart, Grace Tiao, Jeremiah A. Wala, Amaro Taylor-Weiner, Mara Rosenberg, Andrew J. Dunford, Manaswi Gupta, Marcin Imielinski, Matthew Meyerson, Rameen Beroukhim, Gad Getz. MuSE Pipeline: Yu Fan, Wenyi Wang. Consensus somatic SNV/indel annotation: Andrew Menzies, Matthias Schlesner, Juri Reimand, Priyanka Dhingra, Ekta Khurana. Somatic SNV, indel merging: major contribution from L. Jonathan Dursi; further contributions from Christina K. Yung, Matthew H. Bailey, Gordon Saksena, Keiran M. Raine, Ivo Buchhalter, Kortine Kleinheinz, Matthias Schlesner, Yu Fan, David Torrents, Matthias Bieg, Paul C. Boutros, Ken Chen, Zechen Chong, Kristian Cibulskis, Oliver Drechsel, Roland Eils, Robert S. Fulton, Josep L. Gelpi, Mark Gerstein, Santiago Gonzalez, Gad Getz, Ivo G. Gut, Faraz Hach, Michael Heinold, Taobo Hu, Vincent Huang, Barbara Hutter, Hyung-Lae Kim, Natalie Jager, Jongsun Jung, Sushant Kumar, Yogesh Kumar, Christopher Lalansingh, Ignaty Leshchiner, Ivica Letunic, Dimitri Livitz, Eric Z. Ma, Yosef E. Maruvka, R. Jay Mashl, Michael D. McLellan, Ana Milovanovic, Morten Muhlig Nielsen, Brian D. O'Connor, Stephan Ossowski, Nagarajan Paramasivam, Jakob Skou Pedersen, Marc D. Perry, Montserrat Puiggros, Romina Royo, Esther Rheinbay, S. Cenk Sahinalp, Iman Sarrafi, Chip Stewart, Miranda D. Stobbe, Grace Tiao, Jeremiah A. Wala, Jiayin Wang, Wenyi Wang, Sebastian M. Waszak, Joachim Weischenfeldt, Michael Wendl, Johannes Werner, Zhenggang Wu, Hong Xue, Sergei Yakneen, Takafumi N. Yamaguchi, Kai Ye, Venkata Yellapantula, Junjun Zhang, David A. Wheeler; major contributions from Li Ding, Jared T. Simpson. Somatic SV merging: Joachim Weischenfeldt, Francesco Favero, Yilong Li. Somatic CNA merging: Stefan Dentro, Jeff Wintersinger, Ignaty Leshchiner. Oxidative artefact filtration: Dimitri Livitz, Ignaty Leshchiner, Chip Stewart, Esther Rheinbay, Gordon Saksena, Gad Getz. Strand bias filtration: Matthias Bieg, Ivo Buchhalter, Johannes Werner, Matthias Schlesner. miniBAM generation: Jeremiah A. Wala, Gordon Saksena, Rameen Beroukhim, Gad Getz. Germline variant identification from whole-genome sequencing: major contributions from Tobias Rausch, Grace Tiao, Sebastian M. Waszak, Bernardo RodriguezMartin, Suyash Shringarpure, Dai-Ying Wu; further contributions from Sergei Yakneen, German M. Demidov, Olivier Delaneau, Shuto Hayashi, Seiya Imoto, Nina Habermann, Ayellet V. Segre, Erik Garrison, Andy Cafferkey, Eva G. Alvarez, Alicia L. Bruzos, Jorge Zamora, José María Heredia-Genestar, Francesc Muyas, Oliver Drechsel, L. Jonathan Dursi, Adrian Baez-Ortega Hyung-Lae Kim, Matthew H. Bailey, R. Jay Mashl, Kai Ye, Ivo Buchhalter, Vasilisa Rudneva, Ji Wan Park, Eun Pyo Hong, Seong Gu Heo, Anthony DiBiase, Kuan-lin Huang, Ivica Letunic, Michael D. McLellan, Steven J. Newhouse, Matthias Schlesner, Tal Shmaya, Sushant Kumar, David C. Wedge, Mark H. Wright, Venkata D. Yellapantula, Mark Gerstein, Ekta Khurana, Tomas Marques-Bonet, Arcadi Navarro, Carlos D. Bustamante, Jared T. Simpson, Li Ding, Reiner Siebert, Hidewaki Nakagawa, Douglas F. Easton; led by Stephan Ossowski, Jose M. C. Tubio, Gad Getz, Francisco M. De La Vega, Xavier Estivill, Jan O. Korbel. RNA-sequencing analysis: major contributions from Nuno A. Fonseca, Andre Kahles, Kjong-Van Lehmann, Lara Urban Cameron M. Soulette, Yuichi Shiraishi, Fenglin Liu, Yao He, Deniz Demircioglu, Natalie R. Davidson, Claudia Calabrese, Junjun Zhang, Marc D. Perry, Qian Xiang; further contributions from Liliana Greger, Siliang Li, Dongbing Liu, Stefan G. Stark, Fan Zhang, Samirkumar B. Amin Peter Bailey, Aurelien Chateigner, Isidro Cortes-Ciriano, Brian Craft, Serap Erkek, Milana Frenkel-Morgenstern, Mary Goldman, Katherine A. Hoadley, Yong Hou, Matthew R. Huska, Ekta Khurana, Helena Kilpinen, Jan O. Korbel, Fabien C. Lamaze, Chang Li, Xiaobo Li, Xinyue Li, Xingmin Liu, Maximillian G. Marin, Julia Markowski, Tannistha Nandi, Morten Muhlig Nielsen, Akinyemi I. Ojesina, Qiang Pan-Hammarstrom, Peter J. Park, Chandra Sekhar Pedamallu, Jakob Skou Pedersen, Reiner Siebert, Hong Su, Patrick Tan, Bin Tean Teh, Jian Wang, Sebastian M. Waszak, Heng Xiong, Sergei Yakneen, Chen Ye, Christina Yung, Xiuqing Zhang, Liangtao Zheng, Jingchun Zhu, Shida Zhu, Philip Awadalla, Chad J. Creighton, Matthew Meyerson, B. F. Francis Ouellette, Kui Wu, Huanming Yang; led by Jonathan Goke, Roland F. Schwarz, Oliver Stegle, Zemin Zhang, Alvis Brazma, Gunnar Ratsch, Angela N. Brooks. Clustering of tumour genomes based on telomere maintenance-related features: major contribution from David Haan; led by Lincoln D. Stein, Joshua M. Stuart. Clustered mutational processes in PCAWG major contributions from Jonas Demeulemeester, Maxime Tarabichi, Matthew W. Fittall; led by Peter J. Campbell, Jan O. Korbel, Peter Van Loo. Tumours without detected driver mutations: Esther Rheinbay, Amaro Taylor-Weiner, Radhakrishnan Sabarinathan, Peter J. Campbell, Gad Getz. Panorama of driver mutations in human cancer: major contributions from Radhakrishnan Sabarinathan, Oriol Pich; further contributions from Inigo Martincorena, Carlota Rubio-Perez, Malene Juul, Jeremiah A. Wala, Steven Schumacher, Ofer Shapira, Nikos Sidiropoulos, Sebastian M. Waszak, David Tamborero, Loris Mularoni, Esther Rheinbay, Henrik Hornshoj, Jordi Deu-Pons, Ferran Muinos, Johanna Bertl, Qianyun Guo, Chad J. Creighton, Joachim Weischenfeldt, Jan O. Korbel, Gad Getz, Peter J. Campbell, Jakob Skou Pedersen, Rameen Beroukhim; led by Abel Gonzalez-Perez. Pilot benchmarking, variant consensus development and validation: major contribution from L. Jonathan Dursi; further contributions from Christina K. Yung, Matthew H. Bailey, Gordon Saksena, Keiran M. Raine, Ivo Buchhalter, Kortine Kleinheinz, Matthias Schlesner, Yu Fan, David Torrents, Matthias Bieg, Paul C. Boutros, Ken Chen, Zechen Chong, Kristian Cibulskis, Oliver Drechsel, Roland Eils, Robert S. Fulton, Josep 


\section{Article}

Gelpi, Mark Gerstein, Santiago Gonzalez, Gad Getz, Ivo G. Gut, Faraz Hach, Michael Heinold, Taobo Hu, Vincent Huang, Barbara Hutter, Hyung-Lae Kim, Natalie Jager, Jongsun Jung, Sushant Kumar, Yogesh Kumar, Christopher Lalansingh, Ignaty Leshchiner, Ivica Letunic, Dimitri Livitz, Eric Z. Ma, Yosef E. Maruvka, R. Jay Mashl, Michael D. McLellan, Ana Milovanovic, Morten Muhlig Nielsen, Brian D. O'Connor, Stephan Ossowski, Nagarajan Paramasivam, Jakob Skou Pedersen, Marc D. Perry, Montserrat Puiggros, Romina Royo, Esther Rheinbay, S. Cenk Sahinalp, Iman Sarrafi, Chip Stewart, Miranda D. Stobbe, Grace Tiao, Jeremiah A. Wala, Jiayin Wang, Wenyi Wang, Sebastian M. Waszak, Joachim Weischenfeldt, Michael Wendl, Johannes Werner, Zhenggang Wu, Hong Xue, Sergei Yakneen, Takafumi N. Yamaguchi, Kai Ye, Venkata Yellapantula, Junjun Zhang, David A. Wheeler; led by Li Ding, Jared T. Simpson. Production somatic variant calling on the PCAWG compute cloud: major contributions from Christina K. Yung, Brian D. O'Connor, Sergei Yakneen, Junjun Zhang; further contributions from Kyle Ellrott, Kortine Kleinheinz, Naoki Miyoshi, Keiran M. Raine, Romina Royo, Gordon Saksena, Matthias Schlesner, Solomon I. Shorser, Miguel Vazquez, Joachim Weischenfeldt, Denis Yuen, Adam P. Butler, Brandi N. Davis-Dusenbery, Roland Eils, Vincent Ferretti, Robert L. Grossman, Olivie Harismendy, Youngwook Kim, Hidewaki Nakagawa, Steven J Newhouse, David Torrents; led by Lincoln D. Stein. PCAWG data portals: major contributions from Mary Goldman, Junjun Zhang Nuno A. Fonseca, Isidro Cortes-Ciriano; further contributions from Qian Xiang, Brian Craft, Elena Pineiro-Yanez, Brian D O'Connor, Wojciech Bazant, Elisabet Barrera, Alfonso Munoz, Robert Petryszak, Anja Fullgrabe, Fatima Al-Shahrour, Maria Keays, David Haussler, John Weinstein, Wolfgang Huber, Alfonso Valencia, Irene Papatheodorou, Jingchun Zhu; led by Vincent Ferretti, Miguel Vazquez.

Competing interests Gad Getz receives research funds from IBM and Pharmacyclics and is an inventor on patent applications related to MuTect, ABSOLUTE, MutSig, MSMuTect, MSMutSig and POLYSOLVER. Hikmat Al-Ahmadie is consultant for AstraZeneca and Bristol-Myers Squibb. Samuel Aparicio is a founder and shareholder of Contextual Genomics. Pratiti Bandopadhayay receives grant funding from Novartis for an unrelated project. Rameen Beroukhim owns equity in Ampressa Therapeutics. Andrew Biankin receives grant funding from Celgene, AstraZeneca and is a consultant for or on advisory boards of AstraZeneca, Celgene, Elstar Therapeutics, Clovis Oncology and Roche. Ewan Birney is a consultant for Oxford Nanopore, Dovetail and GSK. Marcus Bosenberg is a consultant for Eli Lilly. Atul Butte is a cofounder of and consultant for Personalis, NuMedii, a consultant for Samsung, Geisinger Health, Mango Tree Corporation, Regenstrief Institute and in the recent past a consultant for 10x Genomics and Helix, a shareholder in Personalis, a minor shareholder in Apple, Twitter, Facebook, Google, Microsoft, Sarepta, 10x Genomics, Amazon, Biogen, CVS, Illumina, Snap and Sutro and has received honoraria and travel reimbursement for invited talks from Genentech, Roche, Pfizer, Optum AbbVie and many academic institutions and health systems. Carlos Caldas has served on the Scientific Advisory Board of Illumina. Lorraine Chantrill acted on an advisory board for AMGEN Australia in the past 2 years. Andrew D. Cherniack receives research funding from Bayer. Helen Davies is an inventor on a number of patent applications that encompass the use of mutational signatures. Francisco De La Vega was employed at Annai Systems during part of the project. Ronny Drapkin serves on the scientific advisory board of Repare Therapeutics and Siamab Therapeutics. Rosalind Eeles has received an honorarium for the GU-ASCO meeting in San Francisco in January 2016 as a speaker, a honorarium and support from Janssen for the RMH FR meeting in November 2017 as a speaker (title: genetics and prostate cancer), a honorarium for an University of Chicago invited talk in May 2018 as speaker and an educational honorarium paid by Bayer \& Ipsen to attend GU Connect 'Treatment sequencing for mCRPC patients with the changing landscape of $\mathrm{mHSPC}^{\prime}$ at a venue at ESMO, Barcelona, on 28 September 2019. Paul Flicek is a member of the scientific advisory boards of Fabric Genomics and Eagle Genomics. Ronald Ghossein is a consultant for Veracyte. Dominik Glodzik is an inventor on a number of patent applications that encompass the use of mutational signatures. Eoghan Harrington is a full-time employee of Oxford Nanopore Technologies and is a stock holder. Yann Joly is responsible for the Data Access Compliance Office (DACO) of ICGC 2009-2018. Sissel Juul is a full-time employee of Oxford Nanopore Technologies and is a stock holder. Vincent Khoo has received personal fees and non-financial support from Accuray, Astellas, Bayer, Boston Scientific and Janssen. Stian Knappskog is a coprincipal investigator on a clinical trial that receives research funding from AstraZeneca and Pfizer. Ignaty Leshchiner is a consultant for PACT Pharma. Carlos López-Otín has ownership interest (including stock and patents) in DREAMgenics. Matthew Meyerson is a scientific advisory board chair of, and consultant for, OrigiMed, has obtained research funding from Bayer and Ono Pharma and receives patent royalties from LabCorp. Serena Nik-Zainal is an inventor on a number of patent applications that encompass the use of mutational signatures. Nathan Pennell has done consulting work with Merck, Astrazeneca, Eli Lilly and Bristol-Myers Squibb. Xose S. Puente has ownership interest (including stock and patents in DREAMgenics. Benjamin J. Raphael is a consultant for and has ownership interest (including stock and patents) in Medley Genomics. Jorge Reis-Filho is a consultant for Goldman Sachs and REPARE Therapeutics, member of the scientific advisory board of Volition RX and Paige.Al and an ad hoc member of the scientific advisory board of Ventana Medical Systems, Roche Tissue Diagnostics, InVicro, Roche, Genentech and Novartis. Lewis R. Roberts has received grant support from ARIAD Pharmaceuticals, Bayer, BTG International, Exact Sciences, Gilead Sciences, Glycotest, RedHill Biopharma, Target PharmaSolutions and Wako Diagnostics and has provided advisory services to Bayer, Exact Sciences, Gilead Sciences, GRAIL, QED Therapeutics and TAVEC Pharmaceuticals. Richard A. Scolyer has received fees for professional services from Merck Sharp \& Dohme, GlaxoSmithKline Australia, Bristol-Myers Squibb, Dermpedia, Novartis Pharmaceuticals Australia, Myriad, NeraCare GmbH and Amgen. Tal Shmaya is employed at Annai Systems. Reiner Siebert has received speaker honoraria from Roche and AstraZeneca. Sabina Signoretti is a consultant for Bristol-Myers Squibb, AstraZeneca, Merck, AACR and $\mathrm{NCl}$ and has received funding from Bristol-Myers Squibb, AstraZeneca, Exelixis and royalties from Biogenex. Jared Simpson has received research funding and travel support from Oxford Nanopore Technologies. Anil K. Sood is a consultant for Merck and Kiyatec, has received research funding from M-Trap and is a shareholder in BioPath.

Simon Tavaré is on the scientific advisory board of Ipsen and a consultant for Kallyope. John F. Thompson has received honoraria and travel support for attending advisory board meetings of GlaxoSmithKline and Provectus and has received honoraria for participation in advisory boards for MSD Australia and BMS Australia. Daniel Turner is a full-time employee of Oxford Nanopore Technologies and is a stock holder. Naveen Vasudev has received speaker honoraria and/or consultancy fees from Bristol-Myers Squibb, Pfizer, EUSA pharma, MSD and Novartis. Jeremiah A. Wala is a consultant for Nference. Daniel J. Weisenberger is a consultant for Zymo Research. Dai-Ying Wu is employed at Annai Systems. Cheng-Zhong Zhang is a cofounder and equity holder of Pillar Biosciences, a for-profit company that specializes in the development of targeted sequencing assays. The other authors declare no competing interests.

Additional information

Supplementary information is available for this paper at https://doi.org/10.1038/s41586-020 1969-6.

Correspondence and requests for materials should be addressed to P.J.C., G.G., J.O.K., J.M.S or L.D.S.

Peer review information Nature thanks Arul Chinnaiyan, Ben Lehner, Nicolas Robine and the other, anonymous, reviewer(s) for their contribution to the peer review of this work. Reprints and permissions information is available at http://www.nature.com/reprints. 


\section{Central data collation}
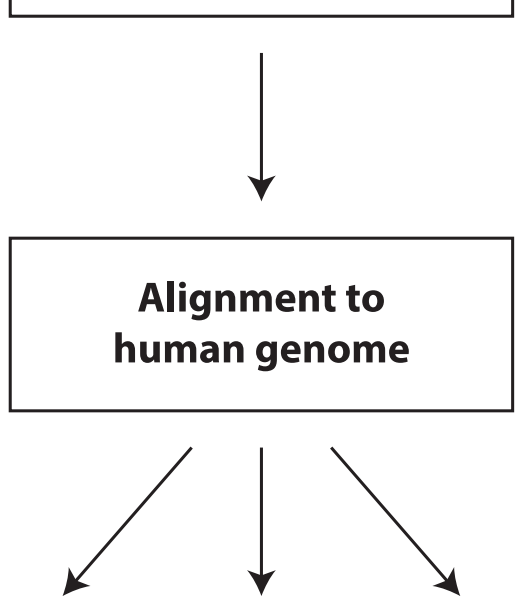

Sanger Inst
pipeline

Whole genome sequencing data

Clinical \& histological data

Genome build hs37d5

Algorithm: BWA-Mem

\section{Broad Inst} pipeline
Call somatic subs, indels, SVs, CNAs Run on cloud computing network Docker images and workflow Available as open source packages

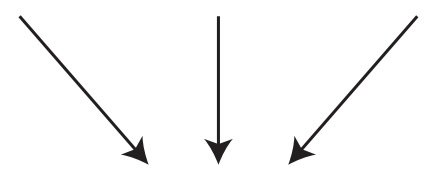

\section{Variant call-set merging}

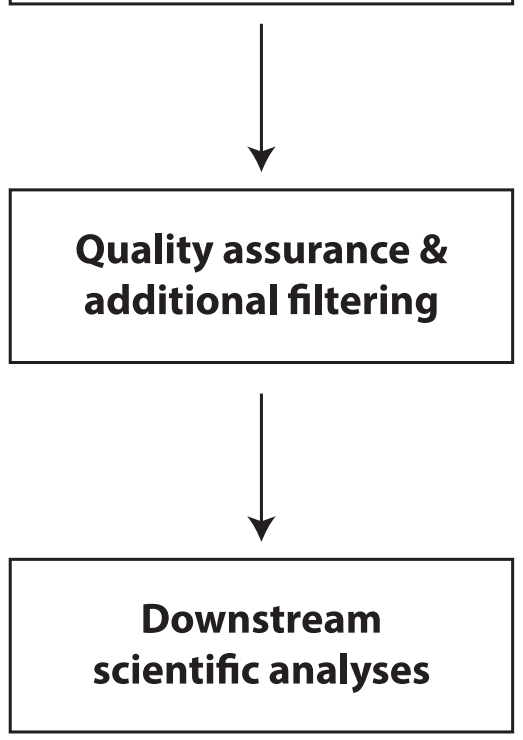

Extended Data Fig. 1 Flow-chart showing key steps in the analysis of PCAWG genomes. After alignment to the genome, somatic mutations were identified by three pipelines, with subsequent merging into a consensus variant set used for downstream scientific analyses. Subs, substitutions; DKFZ/EMBL, the German Cancer Research Centre (DKFZ) and Europen Molecular Biology Laboratory (EMBL).
Merging informed by validation data Indel merging by logistic regression Subs and SVs used 2 of 4 callers

\section{Remove artefacts from oxidative damage and other effects}

16 scientific Working Groups Thematic analyses 
A

Substitutions
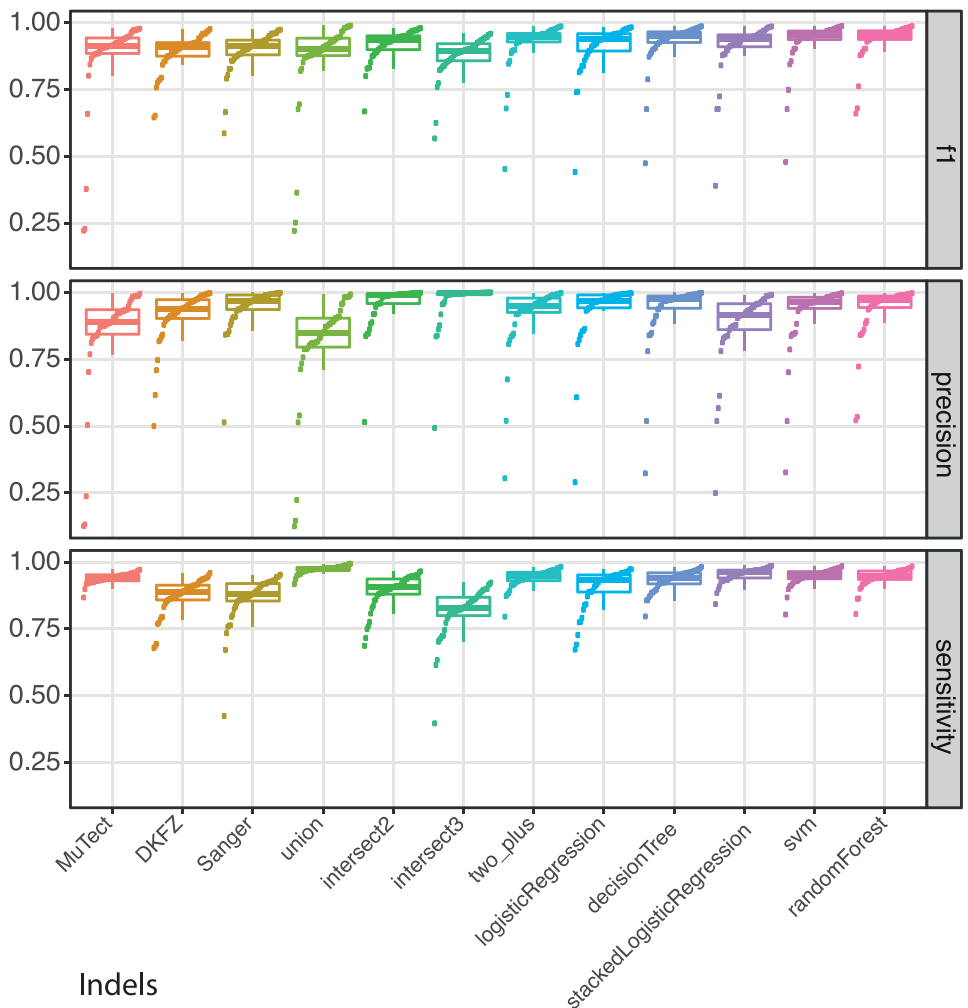

B
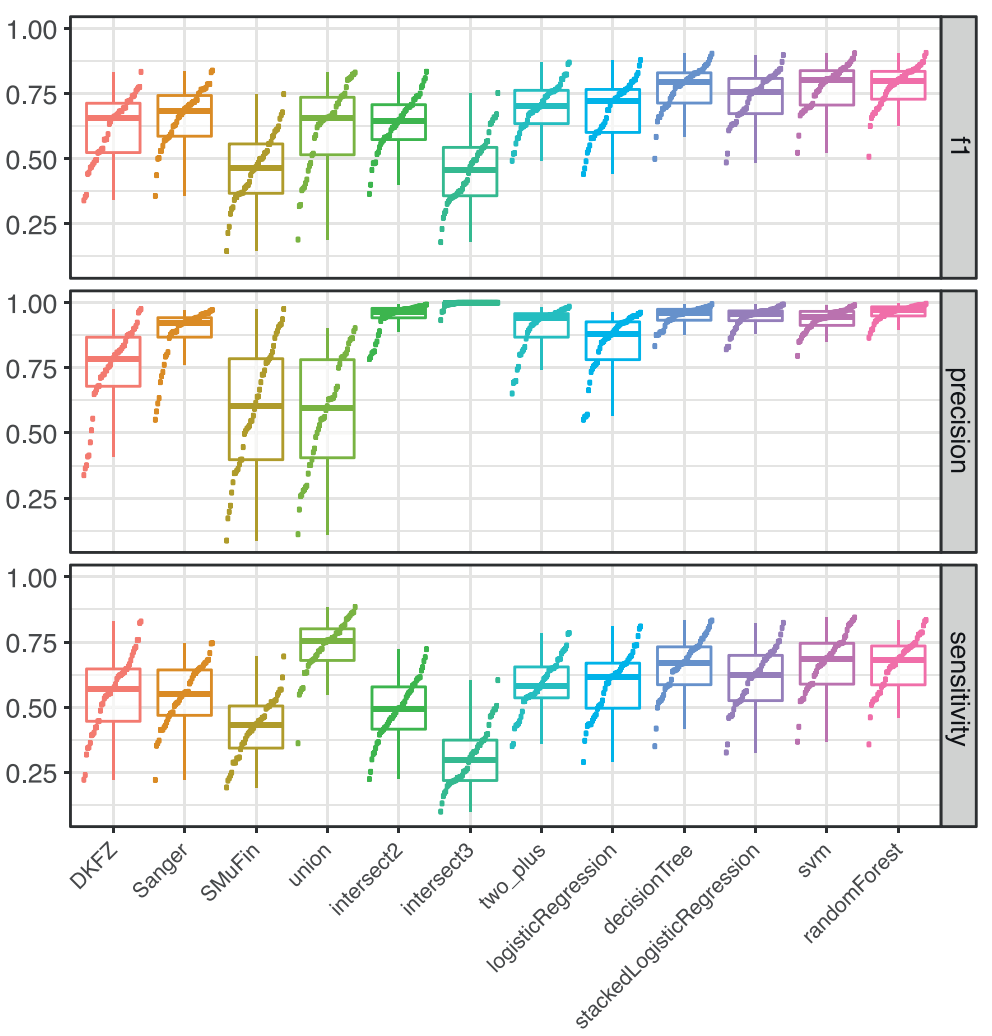

Extended Data Fig. 2 | Distribution of accuracy estimates across algorithms and samples from validation data. a, $F_{1}$ accuracy, precision and sensitivity estimates for somatic SNVs across the core algorithms and different approaches to merging the call-sets. The box plots demarcate the interquartile range and median of estimates across the $n=50$ samples in the validation dataset.b, $F_{1}$ accuracy, precision and sensitivity estimates for somatic indels ( $n=50$ samples). SVM, support vector machine; union, calls made by all variantcalling algorithms; intersect 2 , calls made by any combination of two variantcalling algorithms; intersect 3 , calls made by any three variant-calling algorithms. 

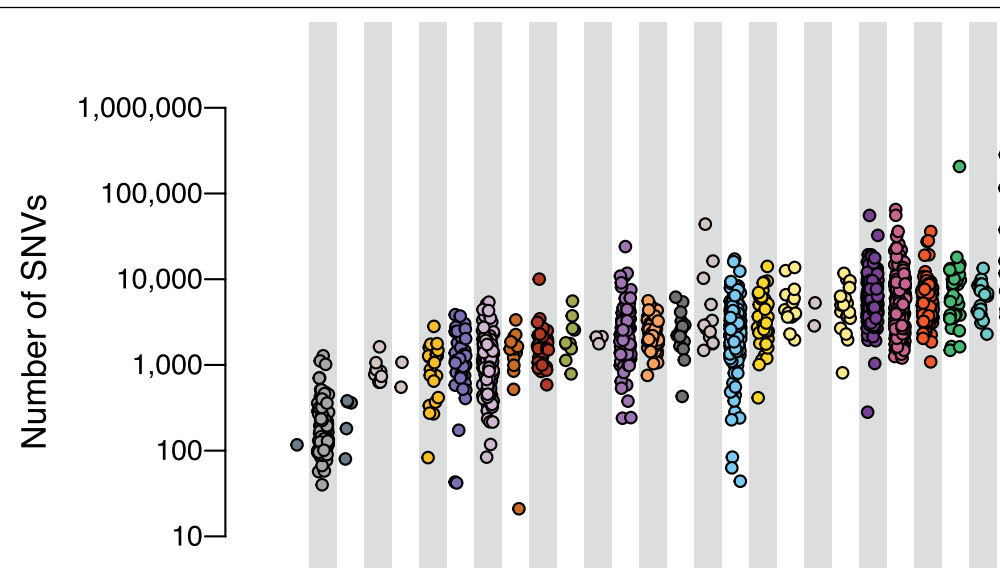

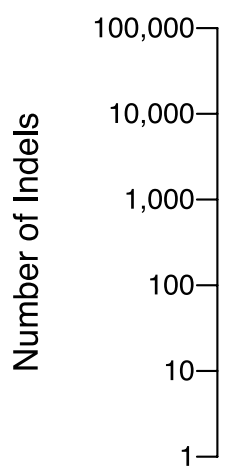

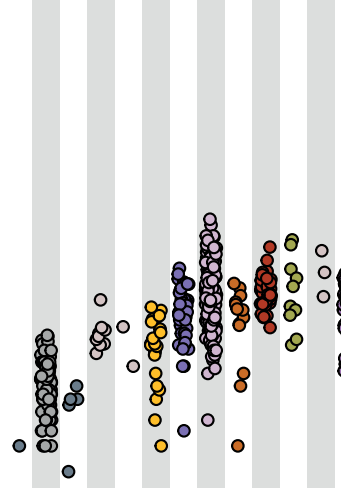

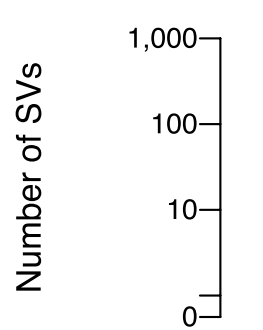

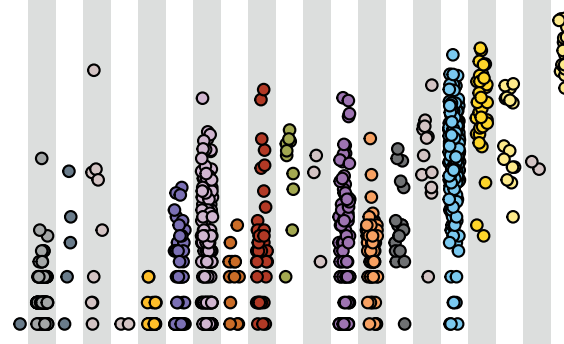

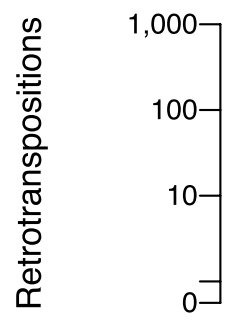
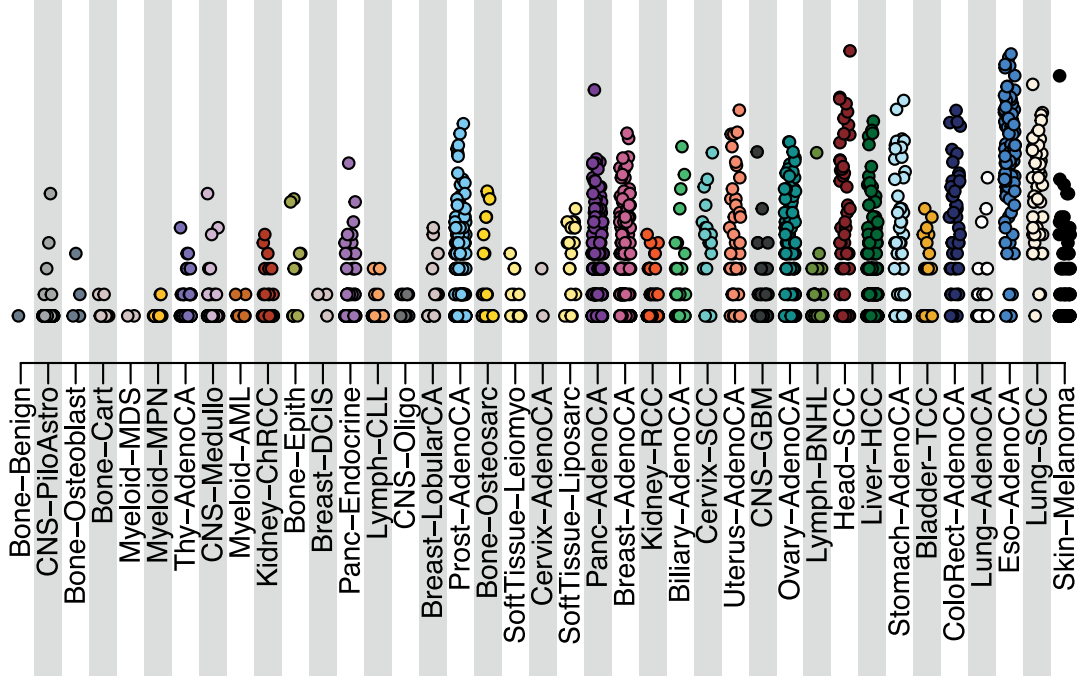

Extended Data Fig. 3 | Distribution of numbers of somatic mutations of different classes across tumour types. The $y$ axis is on a log scale. The 2,583 donors with the highest quality metrics (white-listed donors) are plotted. SNVs indicate substitutions; indels are taken as insertions or deletions $<100 \mathrm{bp}$ in size; retrotranspositions are the combined counts of somatic retrotransposon insertions, transductions and somatic pseudogene insertions. 
A

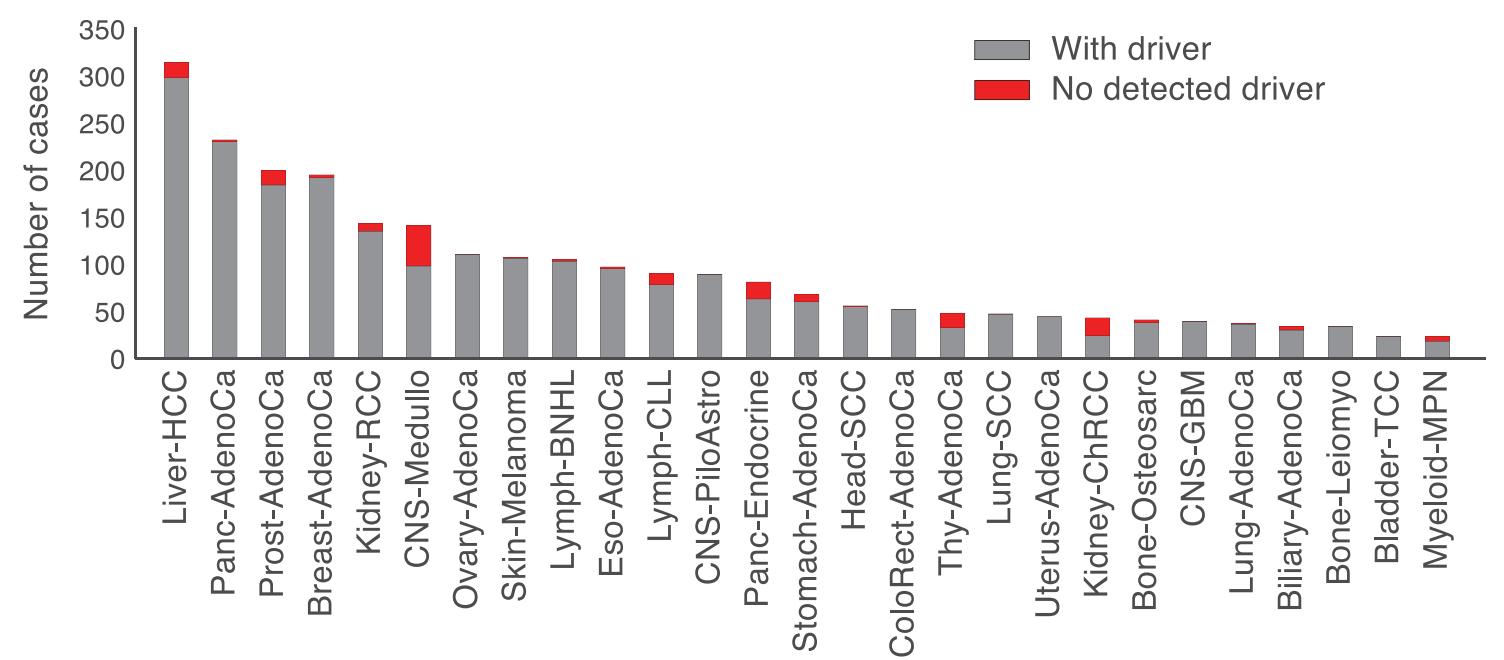

B
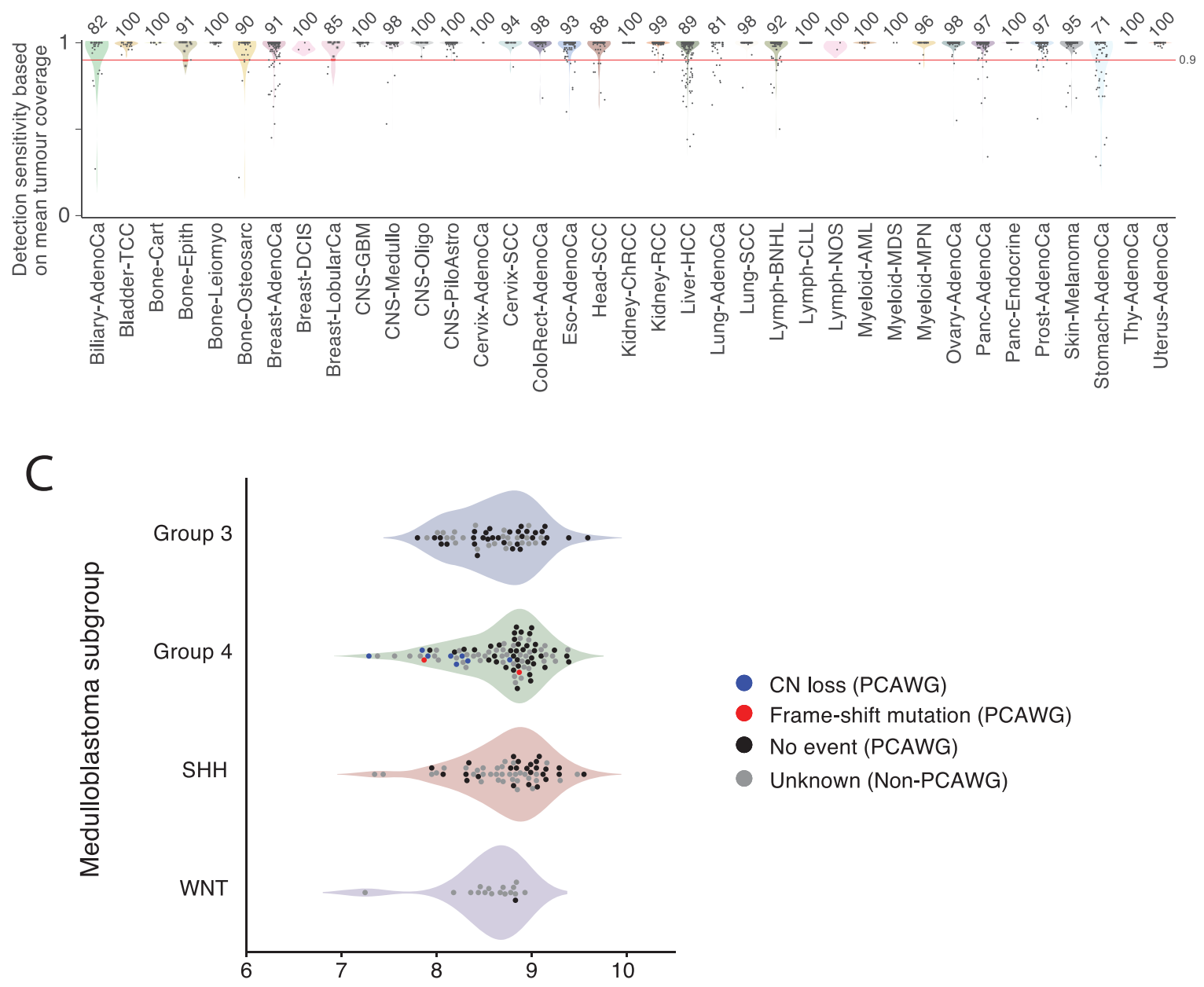

Average SETD2 expression signal (log2)

Extended Data Fig. 4 | Patients with no detected driver mutations in PCAWG. a, Number (red) of patients without detected driver mutations distributed across the different tumour types studied. b, Estimated sensitivity for detecting somatic point mutations genome-wide across tumour types (total sample size: $n=2,583$ patients). Each point represents the estimate for a single patient, layered on violin plots that show the estimated density distribution of sensitivity values for that tumour type (the width proportional is to density). c, SETD2 expression levels across different medulloblastoma subtypes. Points represent individual patients, coloured by whether the gene exhibited focal copy number $(\mathrm{CN})$ loss or a truncating point mutation, or was the wild-type gene. The coloured areas are violin plots showing the estimated density distribution of expression values for that medulloblastoma subtype. 
A Chromoplexy in a thyroid adenocarcinoma

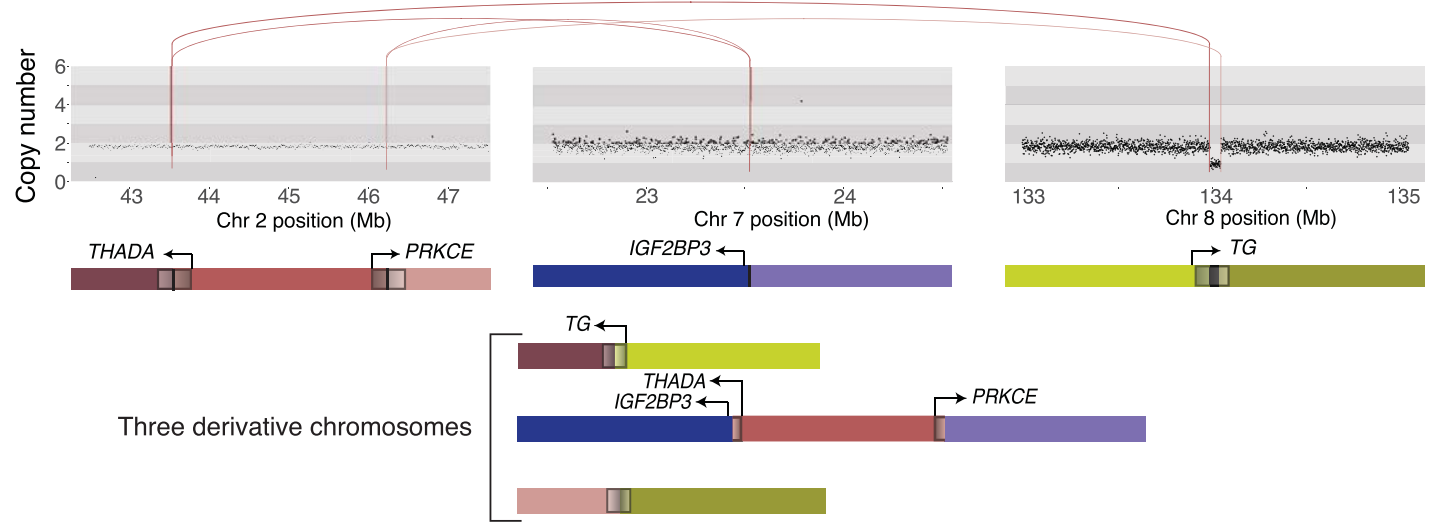

\section{B Kataegis in a pancreatic adenocarcinoma}

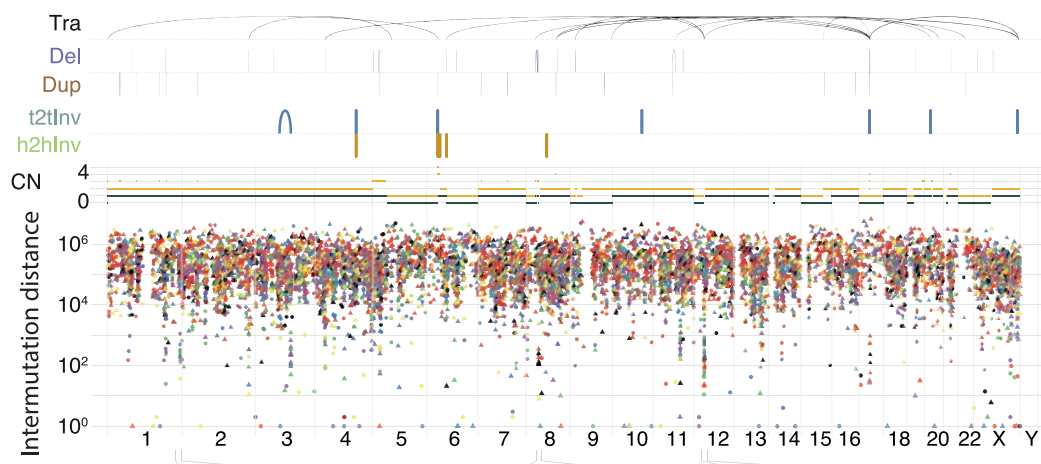

$$
\begin{aligned}
& \text { - } \mathrm{C}>\mathrm{A} \\
& \text { - } \mathrm{C}>\mathrm{G} \\
& \text { - } C>T \\
& \text { - } \mathrm{T}>\mathrm{A} \\
& \text { - } T>C \\
& \text { - } T>G \\
& \text { + strand }
\end{aligned}
$$

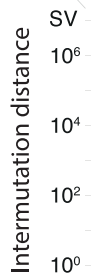
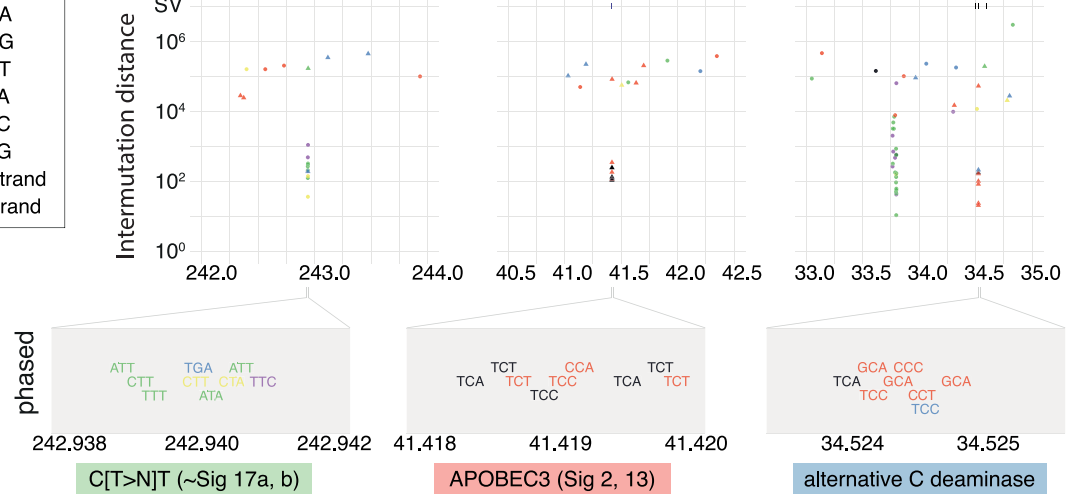

APOBEC3 (Sig 2, 13)

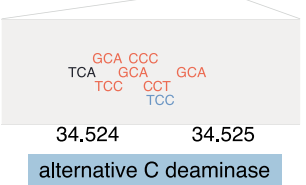

\section{Chromothripsis in a melanoma}

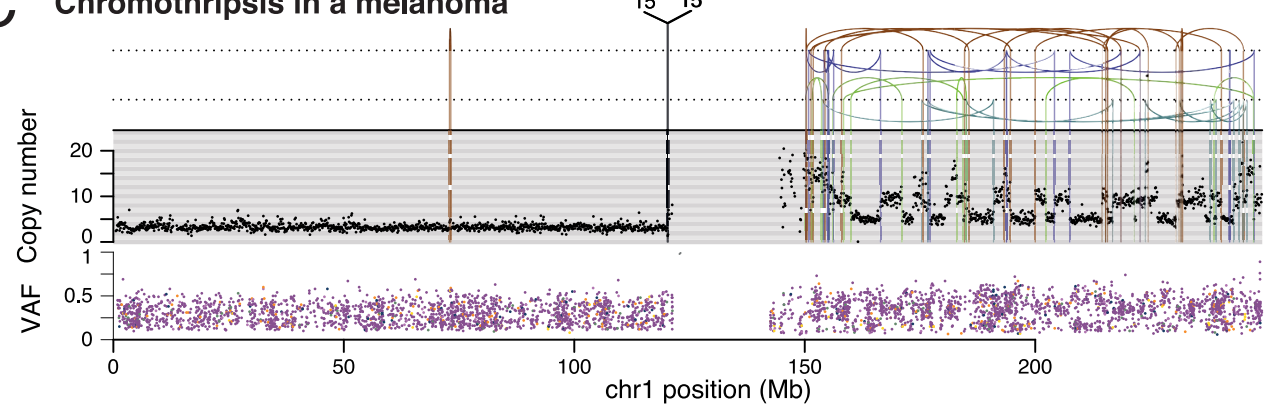

Extended Data Fig. 5 | See next page for caption. 


\section{Article}

Extended Data Fig. 5 | Examples of clustered mutational processes.

a, Chromoplexy example in a thyroid adenocarcinoma. Genes at the

breakpoints are schematically depicted in their normal genomic context and again in the reconstructed derivative chromosomes below. b. Distinct kataegis signatures in the genome of a pancreatic adenocarcinoma sample. SVs and their classification are shown above the main rainfall plot, as well as the total and minor allele copy number. Tra, translocation; del, deletion; dup, duplication; t2tInv, tail-to-tail inversion; h2hInv, head-to-head inversion. Magnifications of the three foci on chromosomes 1, 8 and 12, respectively, highlight distinct manifestations of kataegis. Left, a novel process similar to signature 17 with $\mathrm{T}>\mathrm{N}$ mutations at $\mathrm{CT}$ or TT dinucleotides. Middle, the prototypical APOBEC3A/B type with $\mathrm{C}>\mathrm{T}$ (signature 2 ) and/or $\mathrm{C}>\mathrm{G} / \mathrm{A}$

(signature 13) substitutions at TpC. Right, an alternative cytidine deaminase(s) with a preference for substitutions at $\mathrm{C} / \mathrm{GpC}$. Most of the SNVs in each of these foci can be phased to the same allele and no evidence of anti-phasing is observed.c, Example of a chromothripsis event in a melanoma. The black points (top) represent copy-number estimates from individual genomic bins, with SVs shown as coloured arcs (translocation in black, deletion in purple, duplication in brown, tail-to-tail inversion in cyan, head-to-head inversion in green) that mostly demarcate copy-number changes. The mate chromosomes are displayed above translocations. Bottom, the variant allele fractions of somatic mutations distributed along the relevant chromosomal region. 

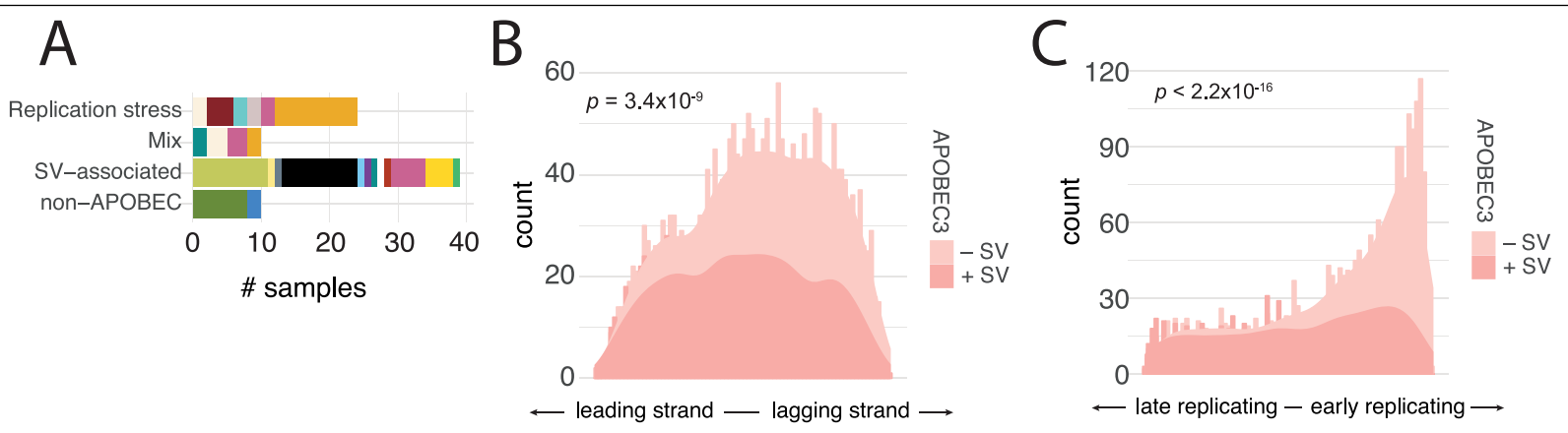

D
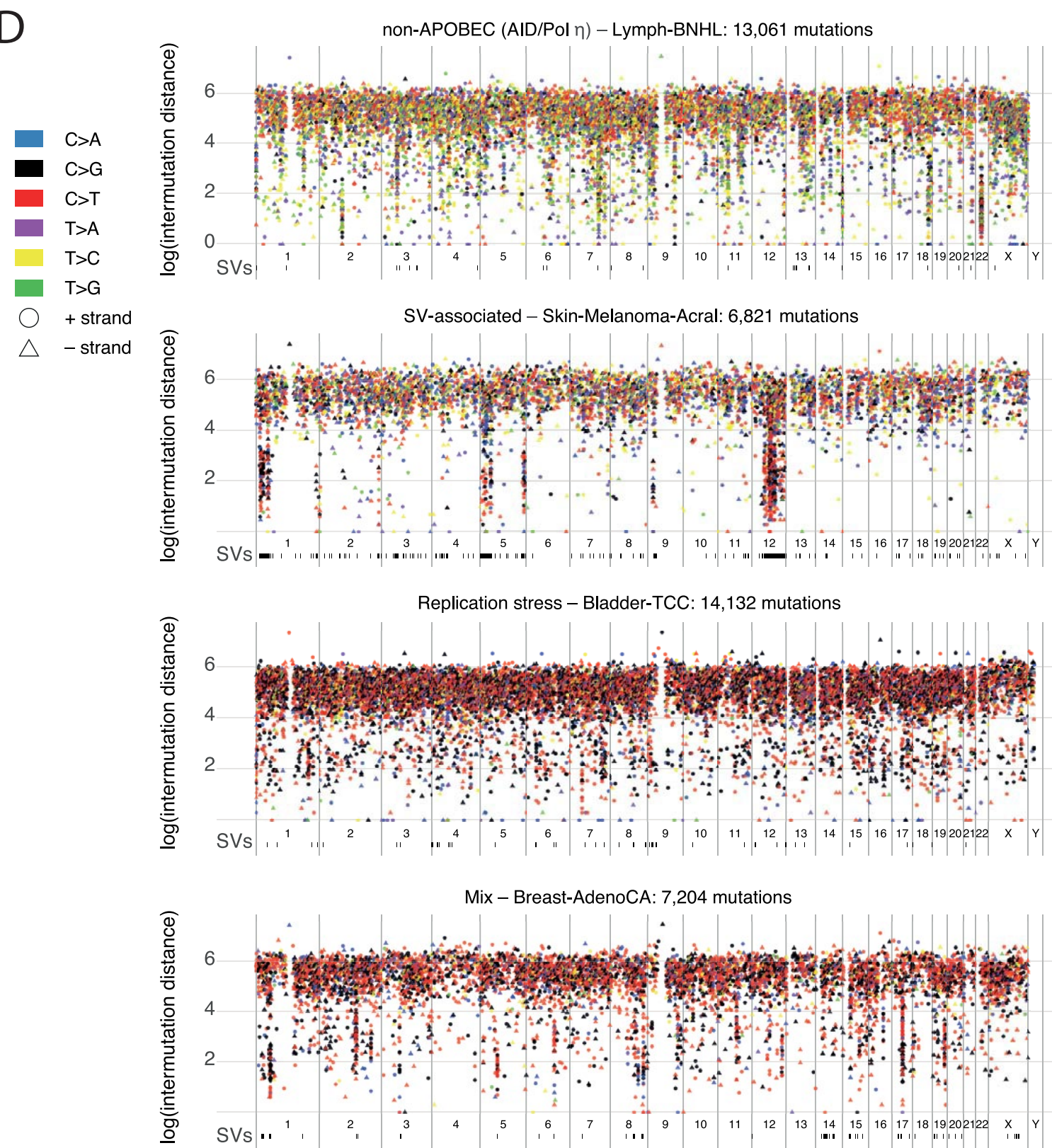

Extended Data Fig. 6 | Patterns of intense kataegis. a, Distribution of the tumour types (colour-coded as in Extended Data Fig. 3) of the samples in the top $5 \%$ of kataegis intensity in each of the four identified genome-wide patterns: non-APOBEC, replication stress, rearrangement-associated and the combination of the last two. b, c, Distribution of leading/lagging strand (b) and replication timing bias (c) for rearrangement-(in)dependent APOBEC kataegis, based on $n=2,583$ tumours. $P$ values were derived using a two-sided MannWhittney $U$-test. d, Example rainfall plots for each of the four identified kataegis patterns. 

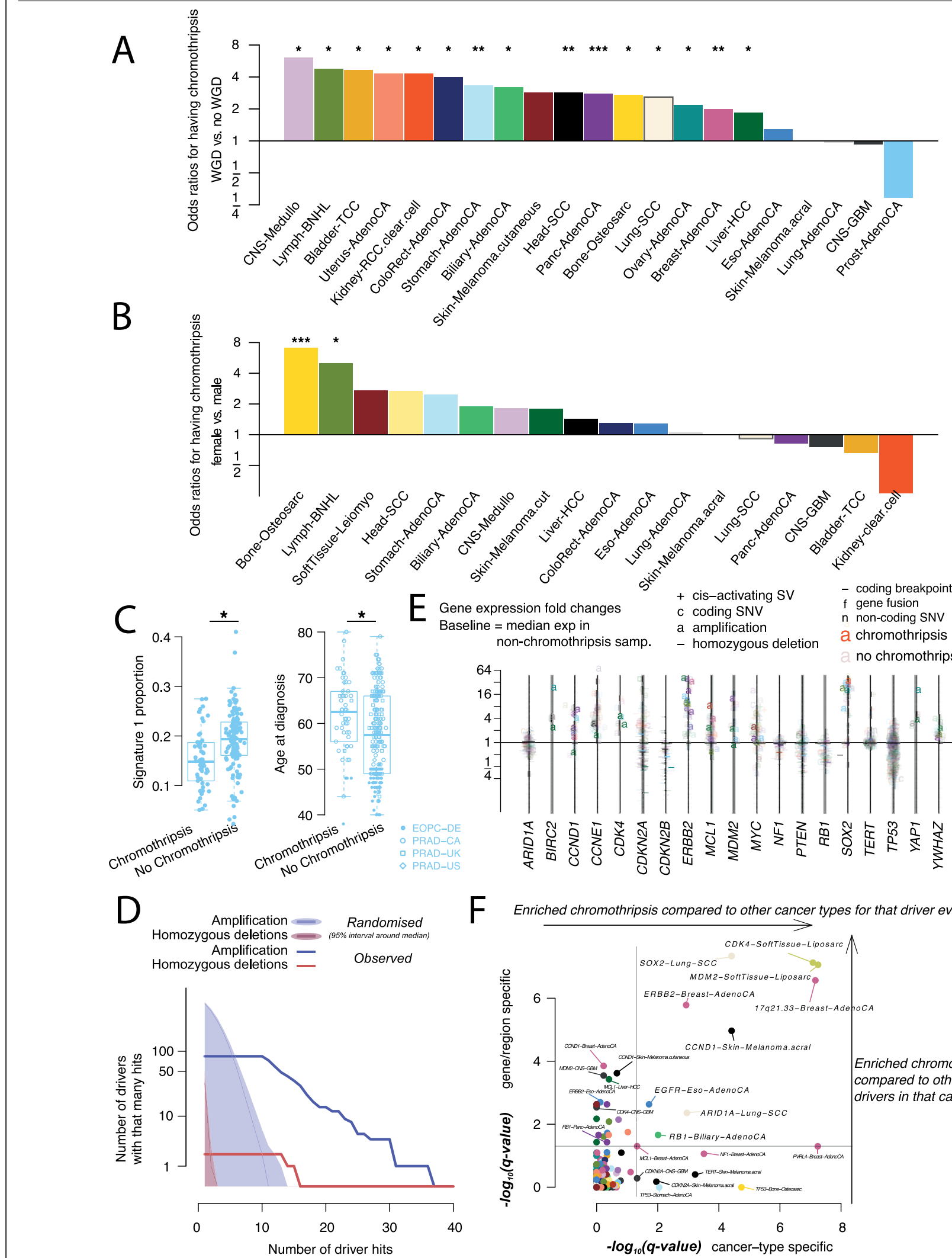

E Enriched chromothripsis compared to other cancer types for that driver event

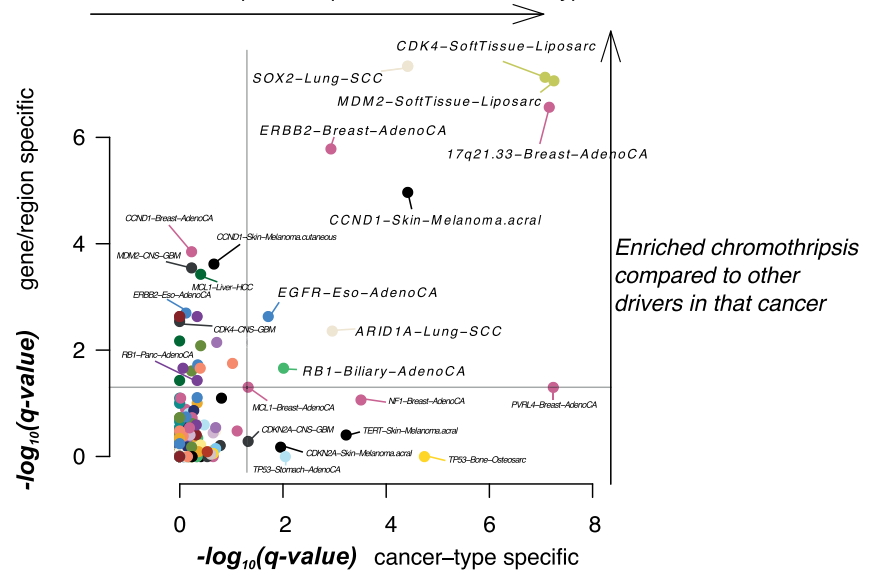

Extended Data Fig. 7 |See next page for caption. 
Extended Data Fig. 7 | Association of chromothripsis with covariates and

driver events. a, Odds ratios per cancer type of containing chromothripsis in whole-genome duplicated versus diploid samples ( $n=2,583$ patients).

${ }^{* * *} q<0.001 ;{ }^{* *} q<0.01 ;{ }^{*} q<0.05$. Two-sided hypothesis testing was performed using Fisher-Boschloo tests, corrected for multiple-hypothesis testing. b, Same as a for female versus male. c, Proportion of mutations explained by single-base substitution signature 1 and age at diagnosis in prostate cancer samples ( $n=210$ patients) with or without chromothripsis $(q<0.05)$. The earlyonset prostate cancer project drives the signal and was sequenced at lower depth. For the box-and-whisker plots, the box denotes the interquartile range, with the median marked as a horizontal line. The whiskers extend as far as the range or $1.5 \times$ the interquartile range, whichever is less. Two-sided hypothesis testing was performed using Mann-Whitney $U$-tests. d, Counts of cooccurrence of chromothripsis with amplification (blue) and homozygous deletions (red) in driver regions: observed (thick line) versus randomized (shaded area and thin line). The cumulative number of drivers that were hit is plotted as a function of the number of times those drivers were hit.e, For each sample in which chromothripsis coincided with a driver event in those genes, we show the fold change in gene expression compared to the median expression of the gene in non-chromothripsis samples of the same cancer type, coloured by cancer type and shaped by the type of driver event. We show with added transparency the fold changes calculated the same way for samples with driver mutations hitting the same driver genes, but that had no evidence of chromothripsis. Analysis is based on $n=1,222$ patients with RNA-sequencing data.f, Enrichment of co-occurrence of chromothripsis with driver events. The $x$ axis shows the association of chromothripsis with a driver in a given cancer type compared with its rate of association with that driver in all other cancer types. The $y$ axis shows the association of chromothripsis with a driver in a given cancer type compared with its rate of association with all other drivers in that type. Exact binomial tests are used and $P$ values are corrected for multiple testing according to the Benjamini-Hochberg method. 
A
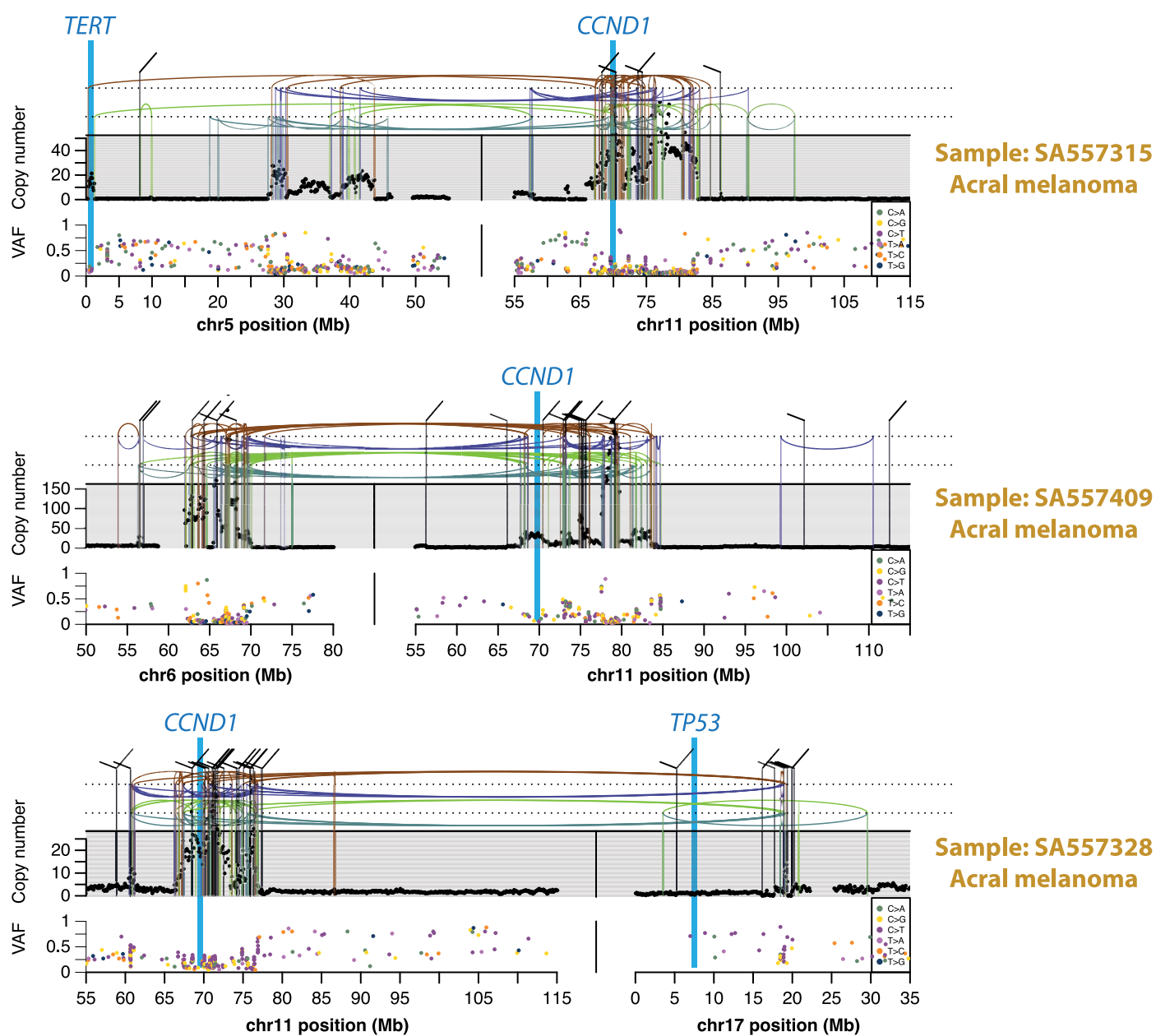

Sample: SA557328

Acral melanoma

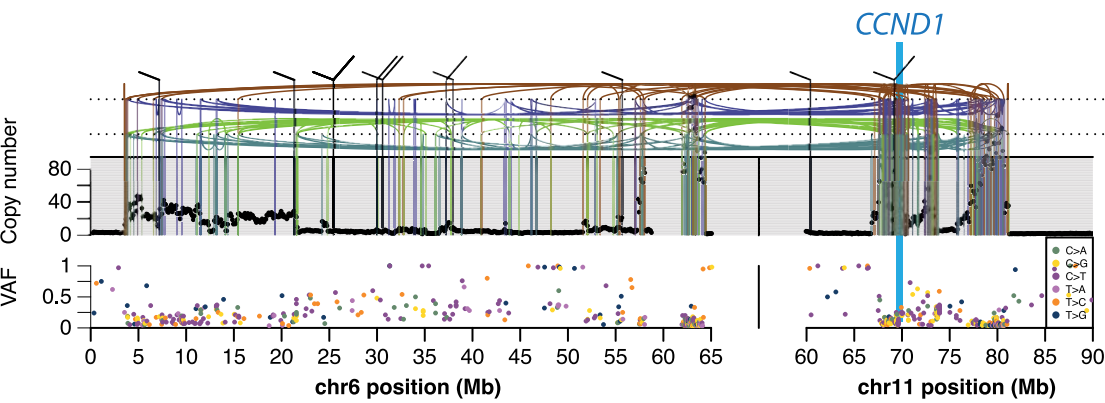

Sample: SA557413

Acral melanoma

B

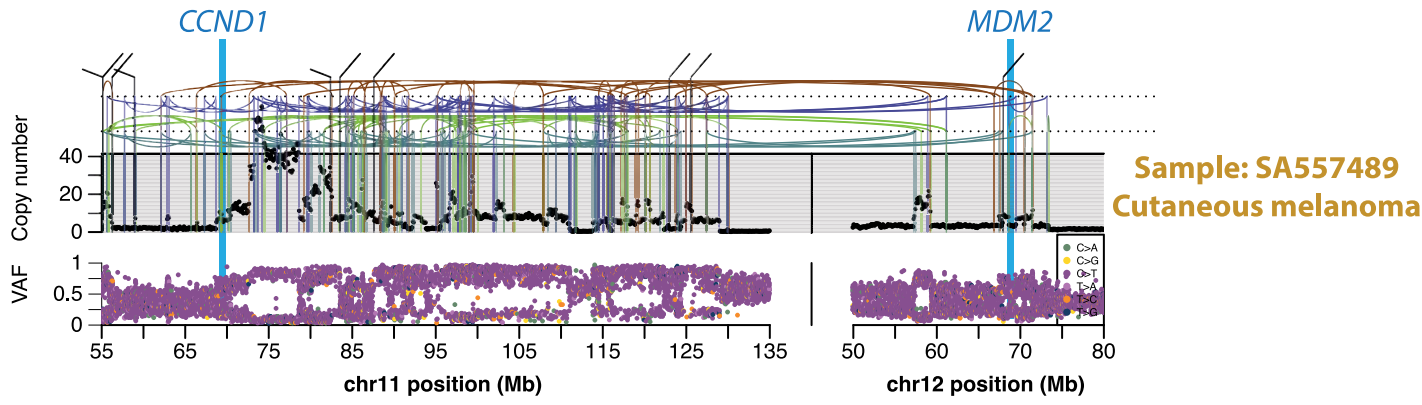

Extended Data Fig. 8 |See next page for caption. 
Extended Data Fig. $8 \mid$ Further examples of chromothripsis-induced amplification targeting multiple cancer-associated genes simultaneously in melanoma. a, Examples of amplifications that occurred early in the development of melanoma. The black points (top) represent copy-number estimates from individual genomic bins, with SVs shown as coloured arcs (translocation in black, deletion in purple, duplication in brown, tail-to-tail inversion in cyan and head-to-head inversion in green) that mostly demarcate copy-number changes. Bottom, the variant allele fractions of SNVs distributed along the relevant chromosomal region. The paucity of somatic mutations at high variant allele fractions in the most-heavily amplified regions indicates that these amplifications began very early in tumour evolution, before the lineage had had opportunity to acquire many SNVs. b, Example of an amplification that occurred late in melanoma development. The large numbers of somatic mutations at high variant allele fractions in the most-heavily amplified regions indicate that these amplifications began late in tumour evolution, after the lineage had already acquired many SNVs. 

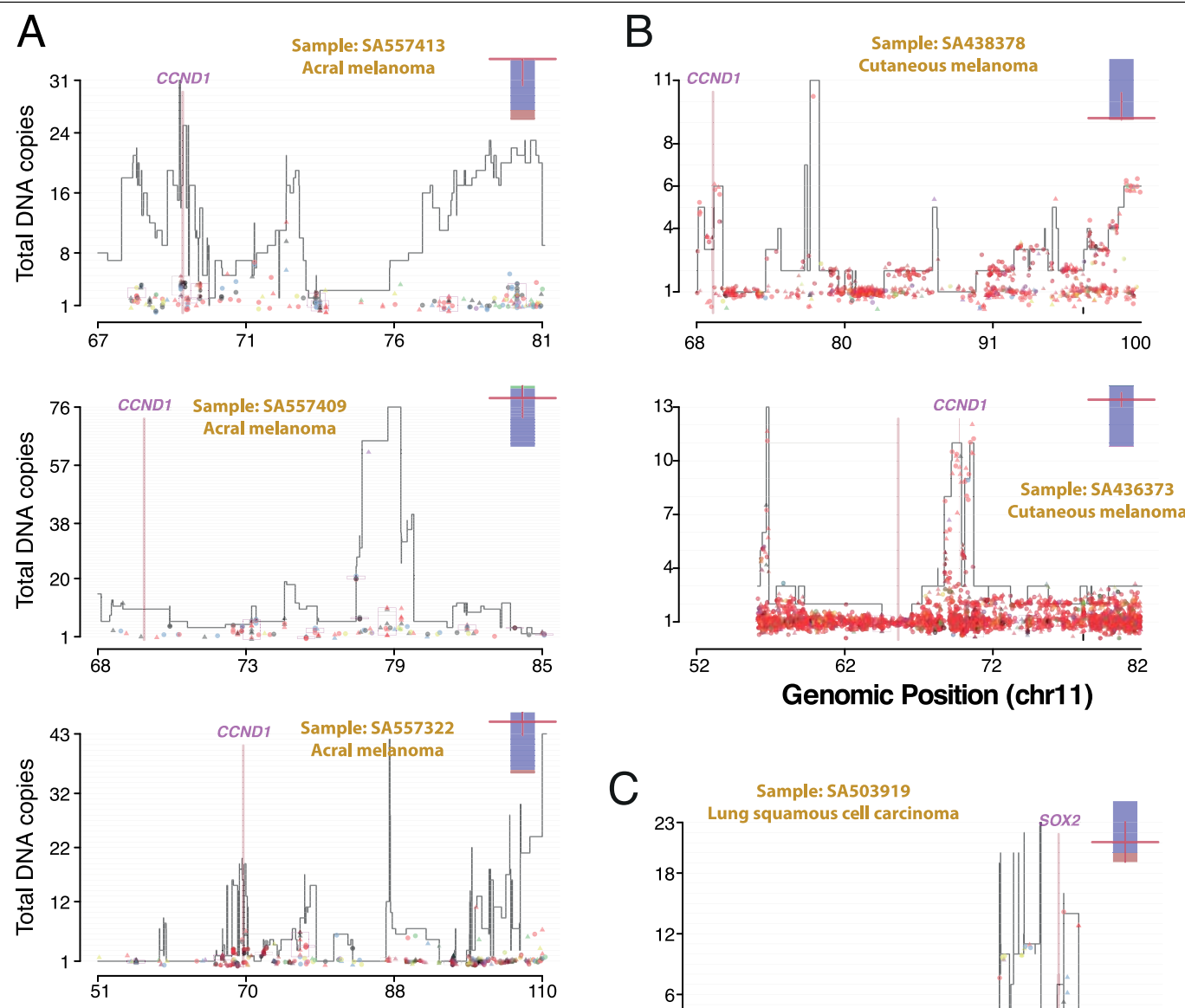

C
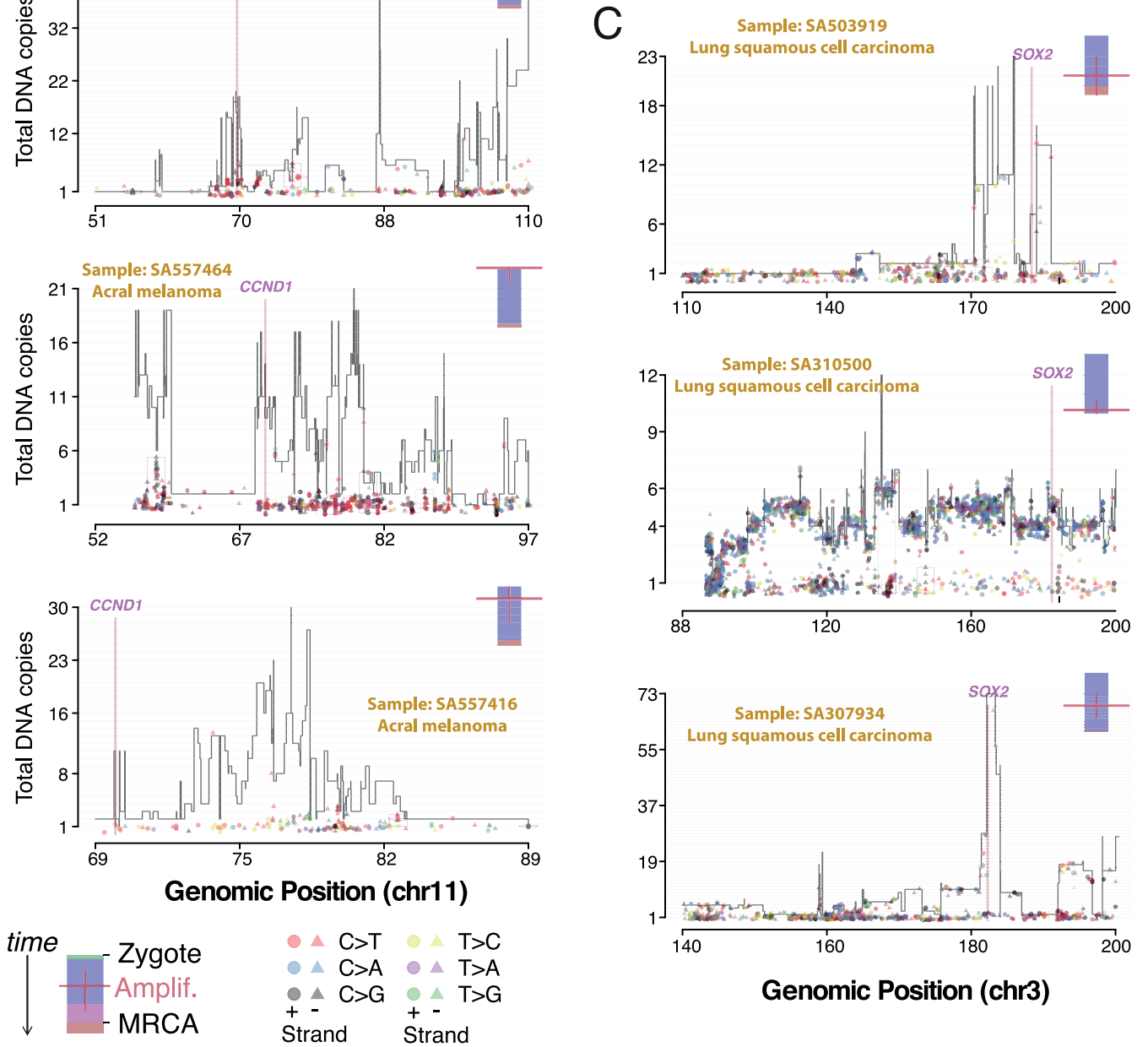

Extended Data Fig. 9|See next page for caption. 
Extended Data Fig. 9 | Timing the amplifications after chromothripsis in molecular time for 10 representative cases. a, Copy-number plot of

chromothriptic regions categorized as 'liposarc-like' in five acral melanomas with $C C N D 1$ amplification. Segments indicate the copy number of the major allele. Points represent SNV multiplicities, that is, the estimated number of copies carrying each SNV, coloured by base change and shaped by strand. Small vertical arrows link SNVs to their corresponding copy-number segment. Kataegis foci are shown within black boxes and show typical strand specificities (all triangles or all circles), similar multiplicities and base changes of signatures 2 and 13 (red and black, respectively). A coloured bar (top right) represents the molecular timing of the amplification (red bar; high is early, low is late) and is coloured by the fraction of total SNVs assigned to the following timing categories: clonal [early], clonal mutations that occurred before duplications involving the relevant chromosome (including whole-genome duplications); clonal [late], clonal mutations that occurred after such duplications; and clonal [NA], mutations that occurred when no duplication was observed. b, Same as a in two cutaneous melanomas, one shows early amplification, the other late amplification. $\mathbf{c}$, Same as a, b, for three lung squamous cell carcinomas and late amplification of SOX2. 
A

Somatic $\mathrm{CpG}$ mutagenesis

(European ancestry; \% Signature 1)

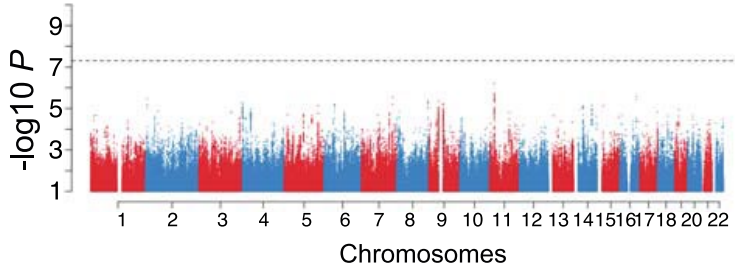

$C$

Somatic APOBEC3B-like mutagenesis (European ancestry)

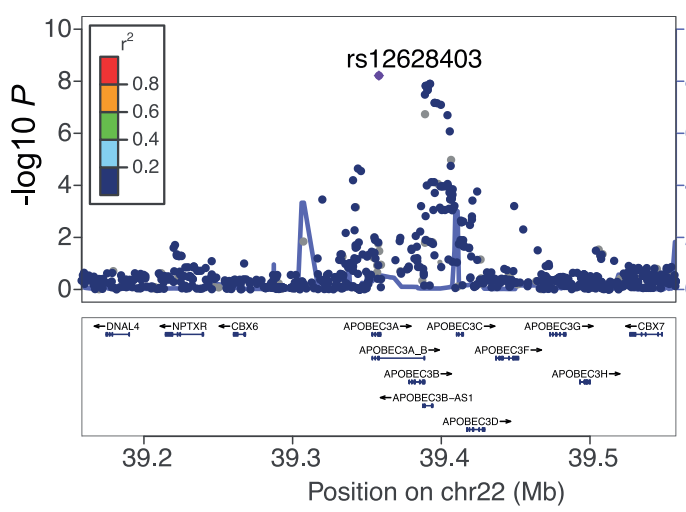

E Somatic APOBEC3B-like mutagenesis (European ancestry; conditional on rs12628403)

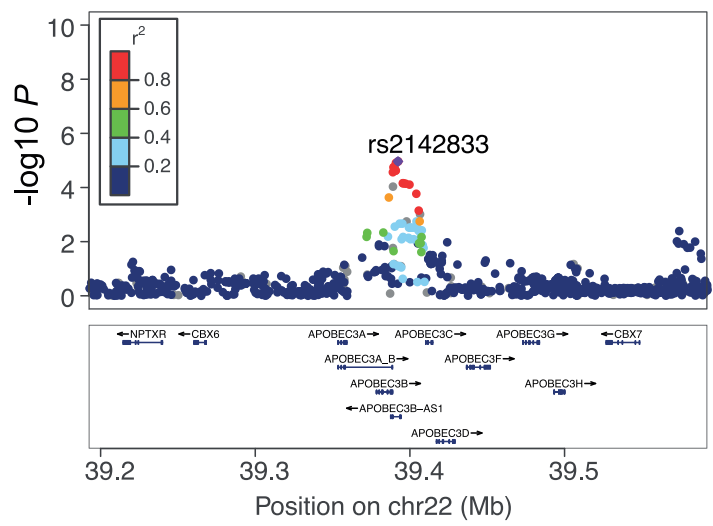

$G$

Pan-cancer association between rs2142833 and APOBEC3[A-G] expression

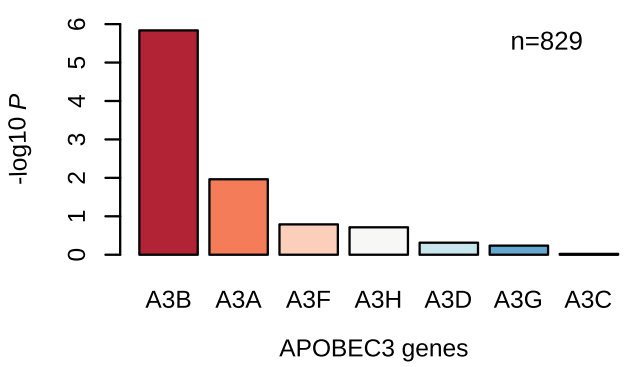

Somatic $\mathrm{CpG}$ mutagenesis

(European ancestry; \% Np[C>T]pG min. estimate)

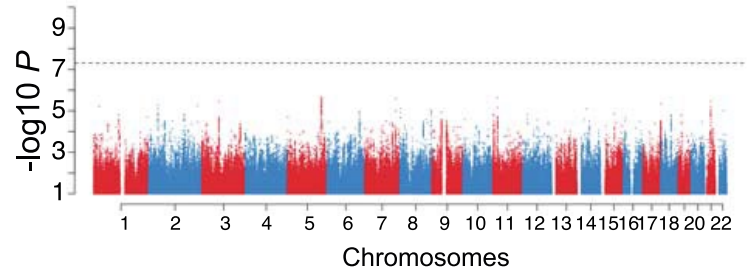

Somatic APOBEC3B-like mutagenesis

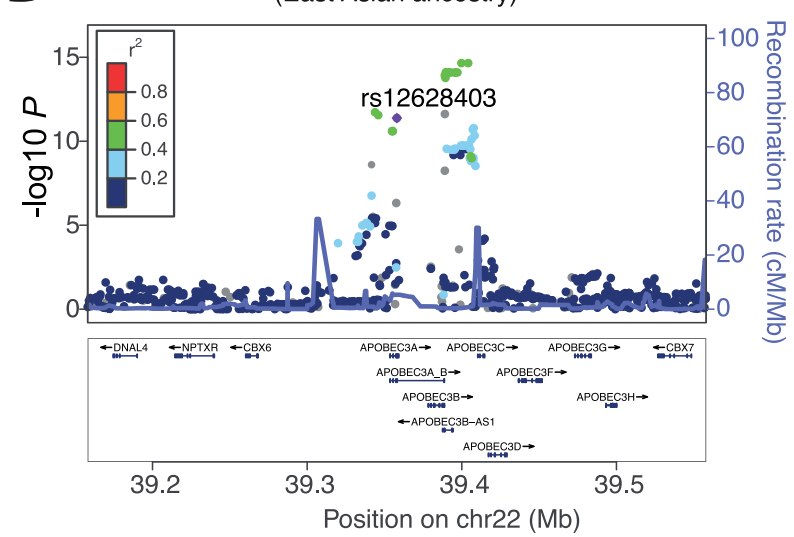

F Somatic APOBEC3B-like mutagenesis (East Asian ancestry; conditional on rs12628403)

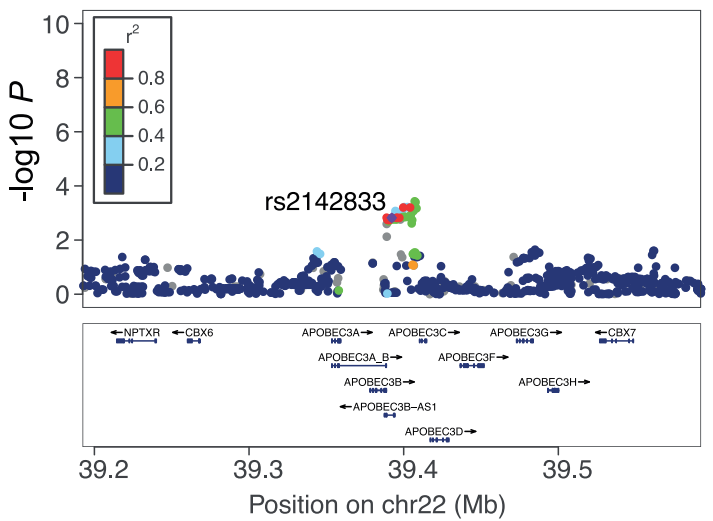

$\mathrm{H}$

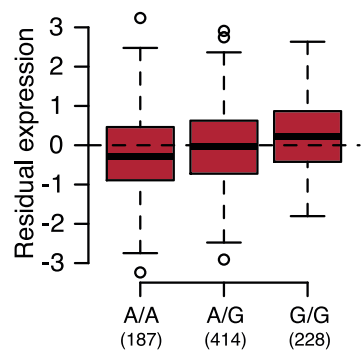

Extended Data Fig. 10 |See next page for caption. 
Extended Data Fig. $10 \mid$ Association between common germline variants and endogenous mutational processes. Genome-wide association of somatic $\mathrm{CpG}$ mutagenesis in individuals of European ancestry ( $n=1,201$ patients) based on mutational signature analysis (a) and NpCpG motif analysis (b). Two-sided hypothesis testing was performed using PLINK v.1.9. To mitigate multiplehypothesis testing, the significance threshold was set to genome-wide significance $\left(P<5 \times 10^{-8}\right)$. c, d, Locuszoom plot for somatic APOBEC3B-like mutagenesis association results, linkage disequilibrium and recombination rates around the genome-wide significant 22q13.1 locus in individuals with European (c) and East Asian (d) ancestry $(n=1,201$ and 318 patients, respectively). Locuszoom plot for somatic APOBEC3B-like mutagenesis association results around the 22q13.1 locus in individuals with European (e) and East Asian (f) ancestry after conditioning on rs12628403. g, h, Association between rs2142833 and expression of $A P O B E C 3$ genes in PCAWG tumour samples (adjusted for sex, age at diagnosis, histology and population structure in linear-regression models with two-sided hypothesis testing not corrected for multiple tests). For the box-and-whisker plot, the box denotes the interquartile range, with the median marked as a horizontal line. The whiskers extend as far as the range or $1.5 \times$ the interquartile range, whichever is less. Outliers are shown as points. 


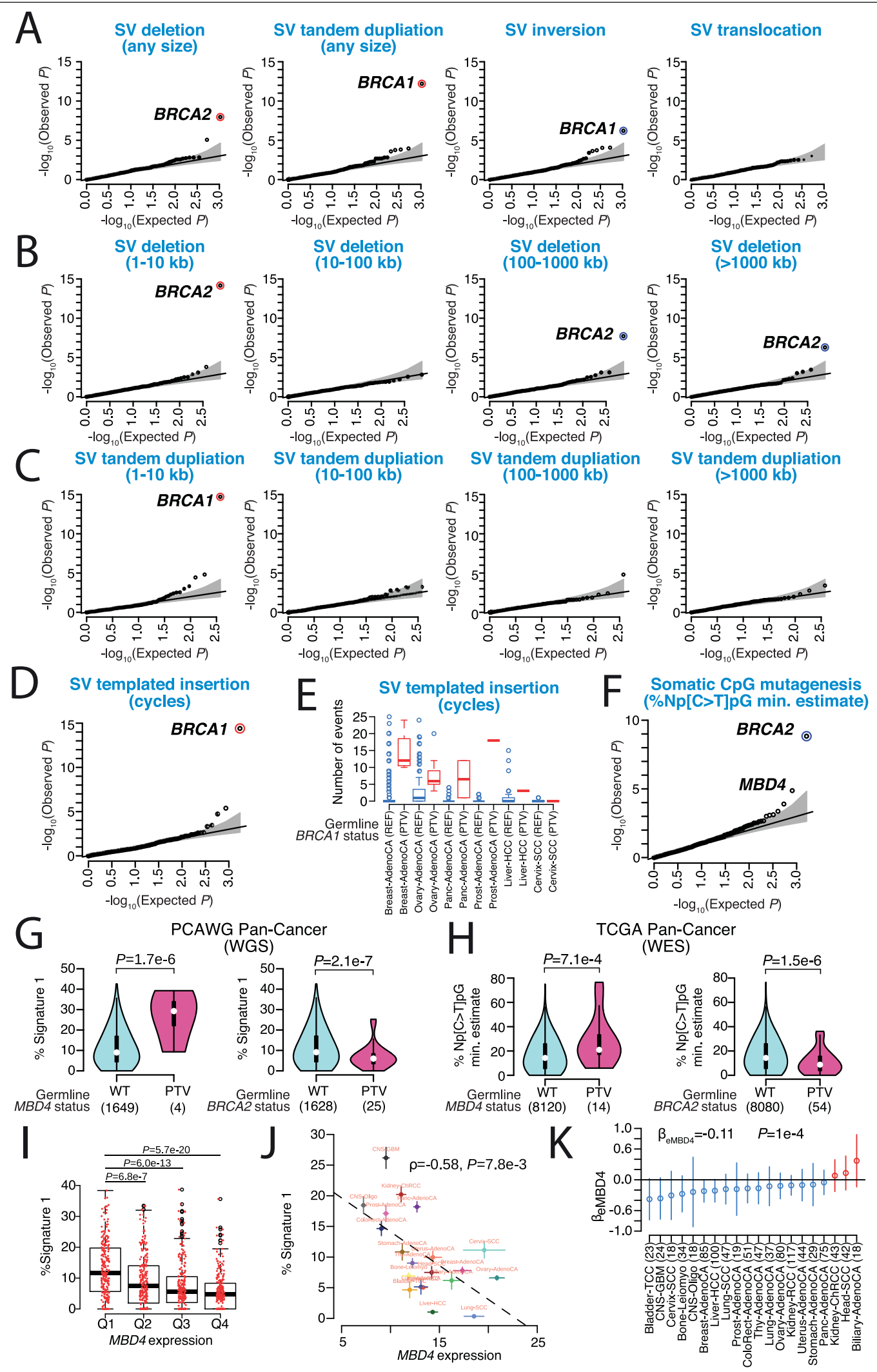

Extended Data Fig. 11 | See next page for caption. 
Extended Data Fig. 11 | Association between rare germline PTVs in proteincoding genes and somatic mutational phenotypes. $\mathbf{a}-\mathbf{d}, \mathbf{f}$, Data are based on two-sided rare-variant association testing across $n=2,583$ patients, with a stringent $P$ value threshold of $P<2.5 \times 10^{-6}$ used to mitigate multiplehypothesis testing (significant genes marked with coloured circles). Blue/red circles mark genes that decrease/increase somatic mutation rates. The black line represents the identity line that would be followed if the observed $P$ values followed the null expectation, with the shaded area showing the $95 \%$ confidence intervals. a, $Q Q$ plots for the proportion of somatic SV deletions, tandem duplications, inversions and translocation in cancer genomes.b, $Q Q$ plots for the proportion of somatic SV deletions in cancer genomes stratified by four size groups (1-10 kb, $10-100 \mathrm{~kb}, 100-1,000 \mathrm{~kb}$ and $>1,000 \mathrm{~kb}) . \mathrm{c}, Q Q$ plots for the proportion of somatic SV tandem duplications in cancer genomes stratified by four size groups (1-10 kb,10-100 kb, 100-1,000 kb and $>1,000 \mathrm{~kb})$. d, $Q Q$ plot for the presence or absence of somatic SV templated insertion (cycles) in cancer genomes. $\mathbf{e}$, Number of SV-templated insertion cycles in PCAWG tumours with germline $B R C A 1$ PTVs. Only histological samples with at least one germline $B R C A 1 \mathrm{PTV}$ carrier are shown ( $n=1,095$ patients combined). The box denotes the interquartile range, with the median marked as a horizontal line. The whiskers extend as far as the range or $1.5 \times$ the interquartile range, whichever is less. Outliers are shown as points. $f, Q Q$ plot for somatic $\mathrm{CpG}$ mutagenesis in cancer genomes based on NpCpG motif analysis. $\mathbf{g}$, Violin plots show estimated densities of the proportion of somatic $\mathrm{CpG}$ mutations in PCAWG donors with germline MBD4 and BRCA2PTVs. The box denotes the interquartile range, with the median marked as a white point. The whiskers extend as far as the range or $1.5 \times$ the interquartile range, whichever is less. Twosided hypothesis testing, not corrected for multiple testing, was performed using linear regression models. h, Replication of germline MBD4 and BRCA2 PTV associations with somatic CpG mutagenesis in TCGA whole-exome sequencing donors. Violin plots show the estimated density of the proportion of somatic CpG mutations in TCGA exomes with germline MBD4 and BRCA2 PTVs. The box denotes the interquartile range, with the median marked as a white point. The whiskers extend as far as the range or $1.5 \times$ the interquartile range, whichever is less. Two-sided hypothesis testing, not corrected for multiple testing, was performed using linear-regression models. i, Correlation between $M B D 4$ expression and somatic $\mathrm{CpG}$ mutagenesis in primary solid PCAWG tumours. Hypothesis testing was two-sided and not corrected for multiple testing, using linear-regression models. The box denotes the interquartile range, with the median marked as a horizontal line. The whiskers extend as far as the range or $1.5 \times$ the interquartile range, whichever is less. j, Data are mean \pm s.e.m. across $n=20$ tumour types. The dashed black line shows the fitted line to the data, estimated using linear-regression models. Hypothesis testing was two-sided and not corrected for multiple testing, using Spearman's rank correlations. k, MBD4 effect sizes (open circles) with $95 \%$ confidence intervals (error bars) for individual cancer types were estimated using linear-regression analysis after (if available) accounting for sex, age at diagnosis (young/old) and ICGC project. Hypothesis testing was two-sided and not corrected for multiple testing. 

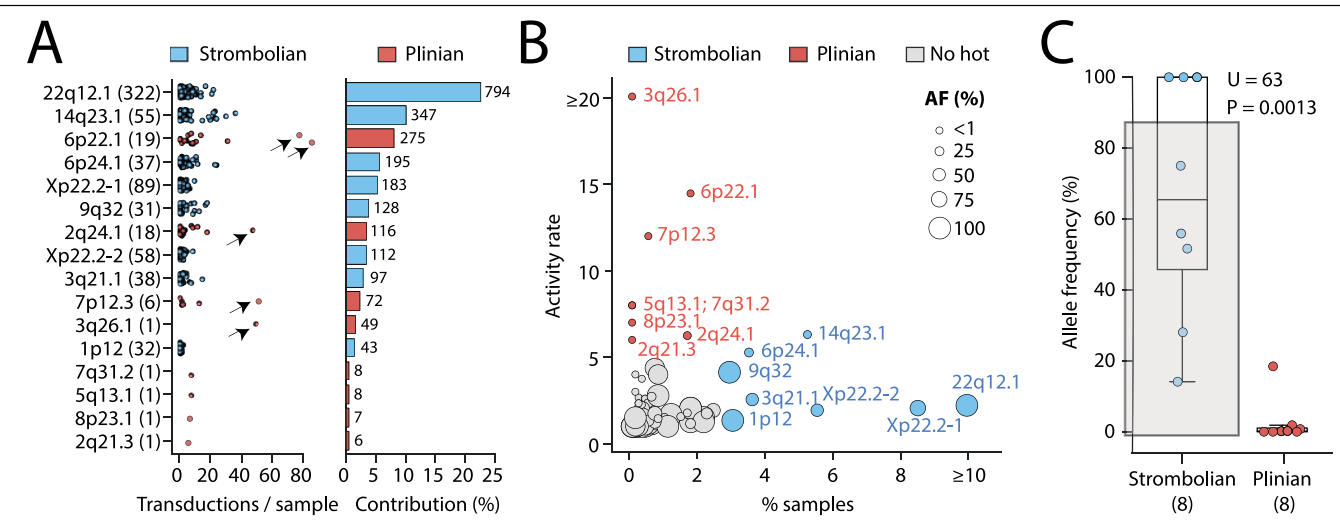

D
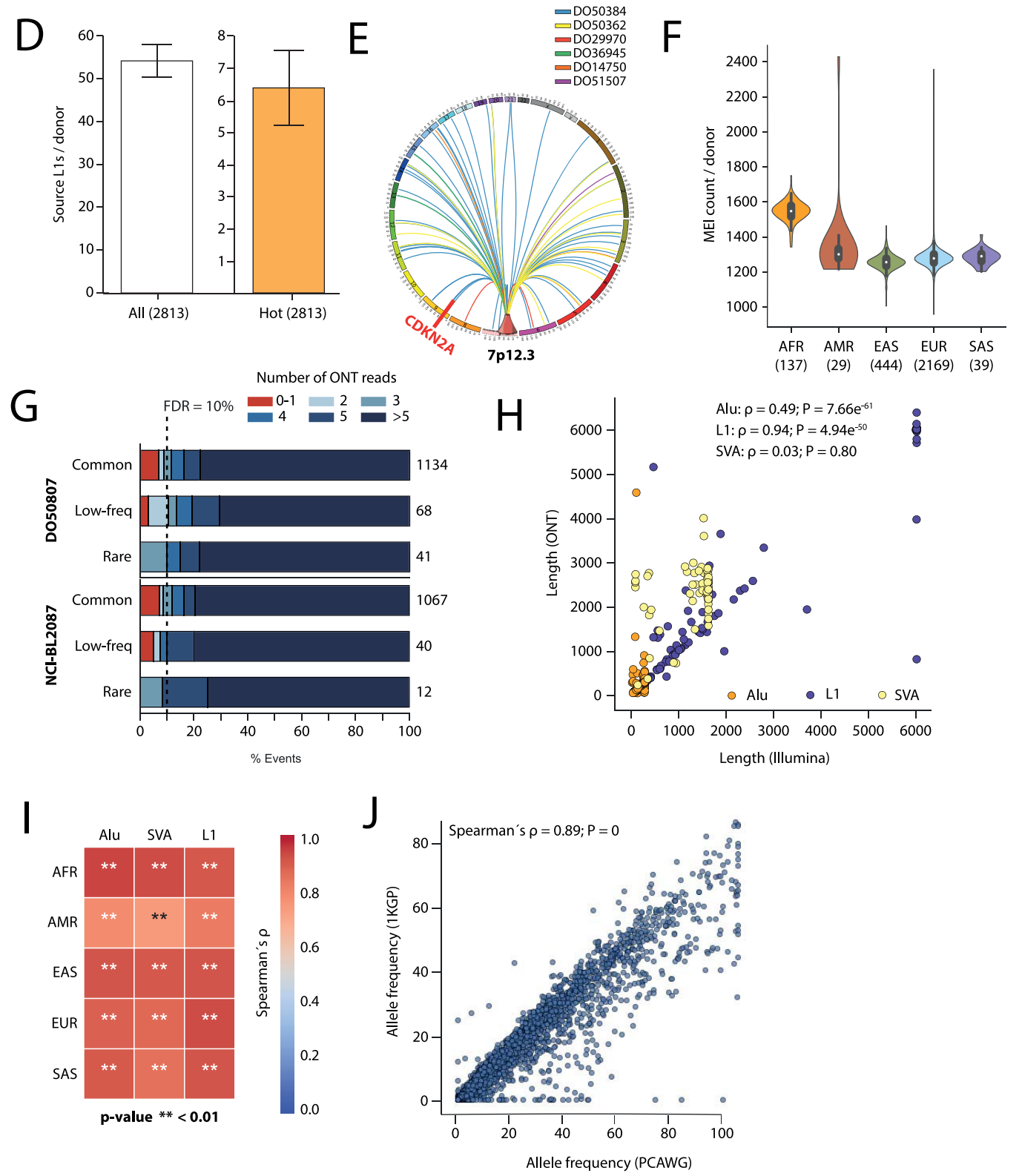

Extended Data Fig. 12 |See next page for caption. 
Extended Data Fig. 12 | Germline MEI call set. a, Left, dots show the number of transductions promoted by each hot element in individual samples. Arrows highlight retrotransposition burst. Right, the contribution of each hot locus is represented. The total number of transductions mediated by each source element is shown on the right. b, Source L1 activity rate (that is, measured as the average number of transductions mediated by an element) versus the percentage of samples with retrotransposition activity in which the germline element is active. For visualization purposes, extreme points observed for a source $L 1$ with an activity rate of 49 and for a L1 active in $31 \%$ of the samples are shown at $\geq 20$ and $\geq 10$, respectively. c, Contrasting allele frequencies for Strombolian and Plinian source loci (sample sizes shown under each axis label). The box denotes the interquartile range, with the median marked as a white point. The whiskers extend as far as the range or $1.5 \times$ the interquartile range, whichever is less. Hypothesis testing was performed using two-sided MannWhitney $U$-tests without correction for multiple tests. d, Numbers of active and hot source $L 1$ elements per donor. Data are mean \pm s.d. number of elements per donor. e, The novel Plinian source element on $7 \mathrm{p} 12.3$ mediates 72 transductions among only 6 cancer samples. This generates a transduction that induces the deletion of the tumour-suppressor gene $C D K N 2 A$.f, Violin plots show the estimated number of distinct germ line MEI alleles per PCAWG donor. The box denotes the interquartile range, with the median marked as a white point. The whiskers extend as far as the range or $1.5 \times$ the interquartile range, whichever is less. Donors are grouped according to their genetic ancestry: AFR, African;
AMR, admixed American; EAS, East Asian; EUR, European; SAS, South Asian. Sample sizes are shown under each axis label. g, For each type of MEI (L1, Blu and SVA) identified both in PCAWG and in the 1000 Genomes Project (1KGP), the correlations between allele frequency estimates per ancestry derived from both projects are displayed in a blue ( 0 ) to red (1) coloured gradient. $n=2,583$ PCAWG patients. Two-sided hypothesis testing was performed using Spearman's rank correlations without correction for multiple tests. h, Example correlation between MEI allele frequencies derived from PCAWG and the 1000 Genomes Project for individuals with European ancestry $(n=1,201$ patients in PCAWG). Two-sided hypothesis testing was performed using Spearman's rank correlations without correction for multiple tests. i, Evaluation of TraFiC-mem false-discovery rate on a liver hepatocellular carcinoma sample (DO50807) and a cell line (NCI-BL2087) sequenced using single-molecule sequencing with MinION (Oxford Nanopore). For each allele frequency bin (common, $>5 \%$; low frequency, $1-5 \%$; rare, $<1 \%$ ), the percentage of events supported by $N$ long reads is represented ( $N$ ranges from $0-1$ to more than 5 ). MEl supported by at least two Nanopore reads were considered to be true positives (blue palette) and were classified as false positives (red) otherwise. The total number of germline MEtIs per allele frequency bin is shown on the rights, Correlation between predicted MEI lengths from Illumine and Nanopore data. Two-sided hypothesis testing was performed using Spearman's rank correlations without correction for multiple testing. 


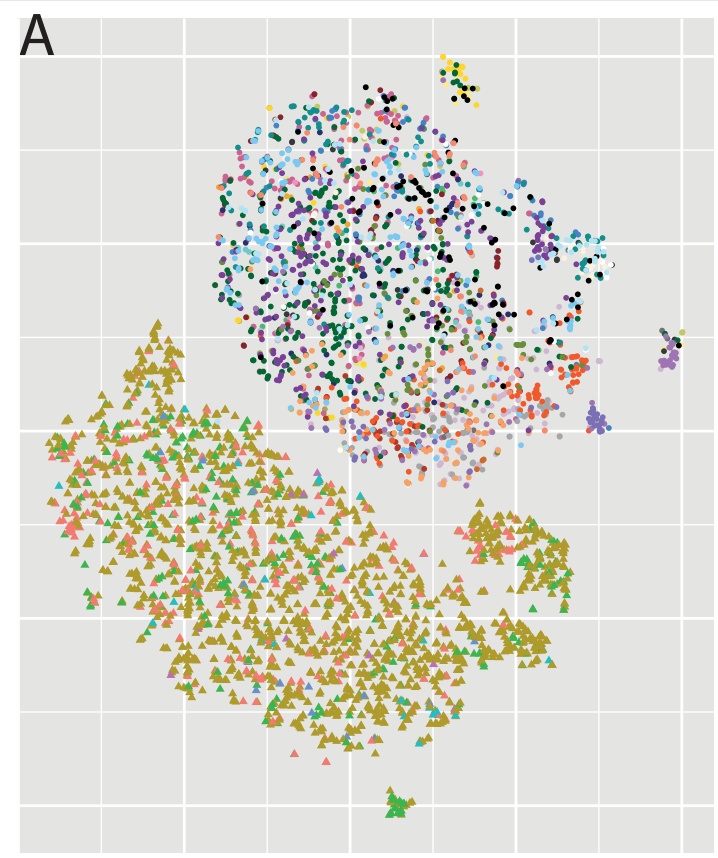
- Biliary-AdenoCA
Lung-SCC
- Bladder-TCC
Lung-AdenoCA
(1) Bone-Osteosarc
- Lymph-BNHL
SoftTissue-Leiomyo
Lymph-CLL
Bone-Benign
- Myeloid-AML
- Bone-Epith
- Ovary-AdenoCA
(2) SoftTissue-Liposarc
- Panc-AdenoCA
- Breast-AdenoCA
- Panc-Endocrine
- Breast-LobularCA
- Cervix-SCC
Prost-AdenoCA
- CNS-Medullo
- CNS-PiloAstro
- CNS-GBM
CNS-Oligo
ColoRect-AdenoCA
Eso-AdenoCA
- Head-SCC
- Skin-Melanoma
Stomach-AdenoCA
- Thy-AdenoCA
- Uterus-AdenoCA
A Normal - solid tissue
A Normal - blood derived
$\triangle$ Normal - tissue adjacent to primary
- Kidney-RCC
$\triangle$ Normal - other
A Normal - bone marrow
- Kidney-ChRCC
A Normal-EBV immortalized

- Liver-HCC

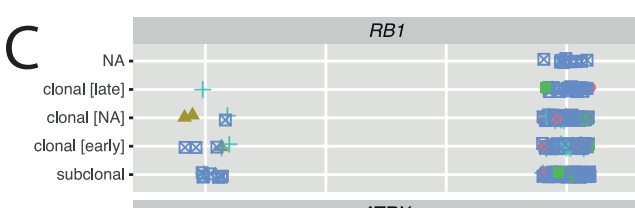

B
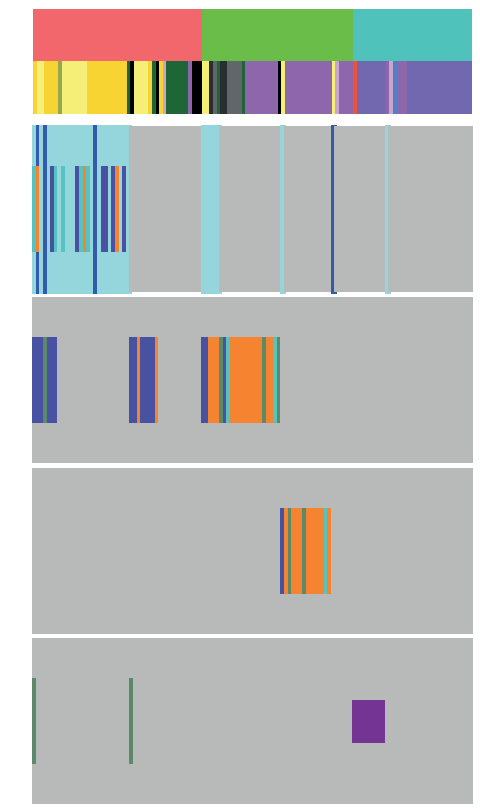

Alterations

Truncating

Deep deletion

Shallow deletion

Missense SNV

Promoter SNV

Splice site

Structural Variant
Tumour type

Bone-Osteosarc

Bone-Leiomyo

Bone-Epith

Liver-HCC

Skin-Melanoma

CNS-Medullo

Panc-Endocrine

CNS-GBM

Thy-AdenoCA

Eso-AdenoCA
Cluster

Tumour type

RB1

ATRX

$D A X X$

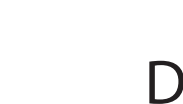

TERT
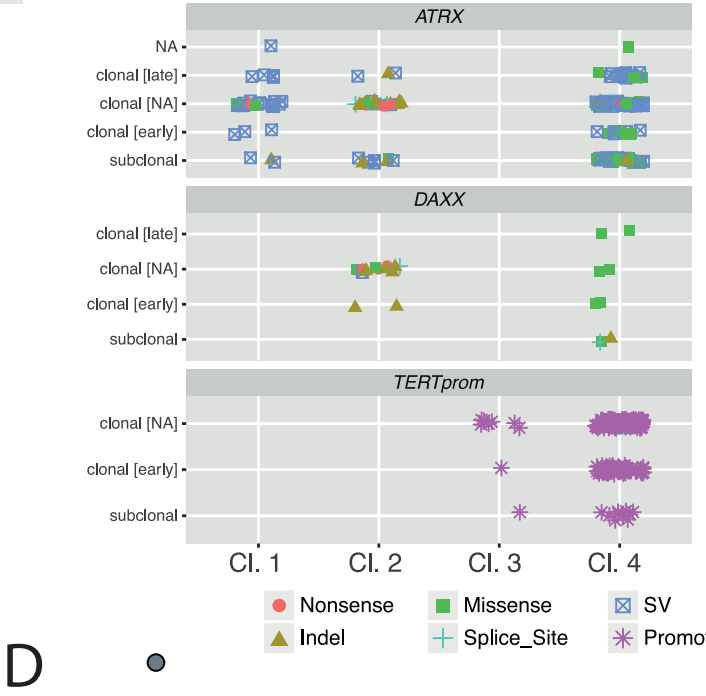

- Nonsense Missense $\square$ SV

$\Delta$ Indel $\quad+$ Splice_Site * Promoter

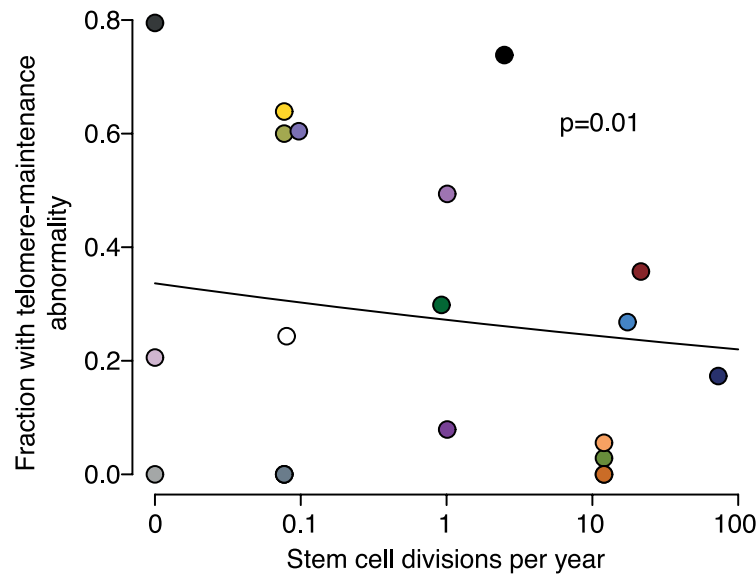

Extended Data Fig. 13 | See next page for caption. 
Extended Data Fig. 13 | Different mechanisms of telomere lengthening in cancer.a, Scatter plot showing the four clusters of tumour-specific telomere patterns identified across PCAWG samples, together with the clusters of matched normal samples, generated by $t$-distributed stochastic neighbour embedding. Circles represent tumour samples and triangles represent matched normal samples. Points are coloured by tissue of origin. Data are based on $n=2,518$ tumour samples and their matched normal samples. b, Patterns of comutation of the relevant driver mutations across individual patients. Columns in plot represent individual patients, coloured by type of abnormality observed.c, Distribution of clonality of driver mutations in genes relevant to telomere maintenance across clusters. Clonal [early], clonal mutations that occurred before duplications involving the relevant chromosome (including whole-genome duplications); clonal [late], clonal mutations that occurred after such duplications; and clonal [NA], mutations that occurred when no duplication was observed. d, Relationship between the estimated number of stem cell divisions per year and rate of telomere maintenance abnormalities across tumour types. The analysis uses data on estimated rates of stem cell division per year across $n=19$ tissue types previously collated from the literature ${ }^{82}$. Tumour types are coloured according to the scheme shown in Extended Data Fig. 3. Two-sided hypothesis testing was performed using likelihood ratio tests on Poisson regression models with no correction for multiple tests. 
Article

Extended Data Table 1 | Overview of the tumour types included in PCAWG project

\begin{tabular}{|c|c|c|c|c|c|c|c|}
\hline \multirow{2}{*}{$\begin{array}{l}\text { Organ } \\
\text { Neural Crest }\end{array}$} & \multirow[t]{2}{*}{ Abbreviation } & \multirow[t]{2}{*}{ Included Subtypes } & \multirow{2}{*}{$\begin{array}{l}\text { Cases } \\
\text { Num. }\end{array}$} & \multicolumn{2}{|c|}{ Sex } & \multicolumn{2}{|c|}{ Age } \\
\hline & & & & $\mathbf{F}$ & M & Med. & $10^{\text {th }}-90^{\text {th }}$ \\
\hline CNS & CNS-GBM & Glioblastoma & 41 & 13 & 28 & 60 & $43-72$ \\
\hline CNS & CNS-Medullo & Medulloblastoma and variants & 146 & 67 & 79 & 9 & $3-28$ \\
\hline CNS & CNS-Oligo & Oligodendroglioma & 18 & 9 & 9 & 41 & $21-62$ \\
\hline CNS & CNS-PiloAstro & Pilocytic astrocytoma & 89 & 47 & 42 & 8 & $2-17$ \\
\hline Skin & Skin-Melanoma & Malignant melanoma & 107 & 38 & 69 & 57 & $37-78$ \\
\hline \multicolumn{8}{|l|}{ Endoderm } \\
\hline Biliary & Biliary-AdenoCA & Papillary cholangiocarcinoma & 34 & 15 & 19 & 64 & $53-76$ \\
\hline Bladder & Bladder-TCC & Transitional cell carcinoma & 23 & 8 & 15 & 65 & $52-80$ \\
\hline Colon/Rectum & ColoRect-AdenoCA & Adenocarcinoma; Mucinous adeno. & 60 & 30 & 30 & 67 & $46-81$ \\
\hline Oesophagus & Eso-AdenoCA & Adenocarcinoma & 98 & 14 & 84 & 70 & $56-79$ \\
\hline Liver & Liver-HCC & Hepatocellular carcinoma; Comb. HCC/cholangio & 317 & 89 & 228 & 67 & $50-78$ \\
\hline Lung & Lung-AdenoCA & Adenocarcinoma; Adenocarcinoma in situ & 38 & 20 & 18 & 66 & $47-77$ \\
\hline Lung & Lung-SCC & Squamous cell carcinoma; Basaloid SCC & 48 & 10 & 38 & 68 & $54-77$ \\
\hline Pancreas & Panc-AdenoCA & Adeno.; Acinar cell Ca.; Mucinous adeno. & 239 & 119 & 120 & 67 & $50-79$ \\
\hline Pancreas & Panc-Endocrine & Neuroendocrine carcinoma & 85 & 30 & 55 & 59 & $38-75$ \\
\hline Prostate & Prost-AdenoCA & Adenocarcinoma & 210 & 0 & 210 & 59 & $47-71$ \\
\hline Stomach & Stomach-AdenoCA & Adenocarcinoma; Mucinous; Papillary; Tubular & 75 & 18 & 57 & 65 & 47-79 \\
\hline Thyroid & Thy-AdenoCA & Adenocarcinoma; Columnar cell; Follicular type & 48 & 37 & 11 & 51 & $26-75$ \\
\hline \multicolumn{8}{|l|}{ Mesoderm } \\
\hline Bone/Soft Tissue & Bone-Benign & Osteoblastoma; Osteofibrous dysplasia & 7 & 4 & 3 & 18 & $12-30$ \\
\hline Bone/Soft Tissue & Bone-Benign & Chondroblastoma; Chrondromyxoid fibroma & 9 & 2 & 7 & 16 & $14-38$ \\
\hline Bone/Soft Tissue & Bone-Epith & Adamantinoma; Chordoma & 10 & 4 & 6 & 60 & $37-67$ \\
\hline Bone/Soft Tissue & Bone-Osteosarc & Osteosarcoma & 38 & 20 & 18 & 20 & $9-58$ \\
\hline Bone/Soft Tissue & SoftTissue-Leiomyo & Leiomyosarcoma & 15 & 10 & 5 & 61 & $51-78$ \\
\hline Bone/Soft Tissue & SoftTissue-Liposarc & Liposarcoma & 19 & 5 & 14 & $\mathrm{n} / \mathrm{a}$ & $\mathrm{n} / \mathrm{a}$ \\
\hline Cervix & Cervix-AdenoCA & Adenocarcinoma & 2 & 2 & 0 & 39 & $33-46$ \\
\hline Cervix & Cervix-SCC & Squamous cell carcinoma & 18 & 18 & 0 & 39 & $25-58$ \\
\hline Head/Neck & Head-SCC & Squamous cell carcinoma & 57 & 10 & 47 & 53 & $34-71$ \\
\hline Kidney & Kidney-ChRCC & Adenocarcinoma, chromophobe type & 45 & 19 & 26 & 47 & $34-69$ \\
\hline Kidney & Kidney-RCC & Clear cell adenocarcinoma; papillary type & 144 & 54 & 90 & 60 & $48-75$ \\
\hline Lymphoid & Lymph-BNHL & Burkitt; Diffuse large B-cell; Follicular; Marginal & 107 & 51 & 56 & 57 & $10-74$ \\
\hline Lymphoid & Lymph-CLL & Chronic lymphocytic leukaemia & 95 & 31 & 64 & 62 & $46-78$ \\
\hline Myeloid & Myeloid-AML & Acute myeloid leukaemia & 10 & 3 & 7 & 50 & $35-56$ \\
\hline Myeloid & Myeloid-MDS & Myelodysplastic syndrome & 2 & 1 & 1 & 76 & $74-77$ \\
\hline Myeloid & Myeloid-MPN & Myeloproliferative neoplasm & 26 & 14 & 12 & 56 & $38-75$ \\
\hline Ovary & Ovary-AdenoCA & Adenocarcinoma; Serous cystadenocarcinoma & 113 & 113 & 0 & 60 & $48-74$ \\
\hline Uterus & Uterus-AdenoCA & Adeno., endometrioid; Serous cystadeno. & 51 & 51 & 0 & 69 & $57-81$ \\
\hline \multicolumn{8}{|l|}{ Ectoderm } \\
\hline Breast & Breast-AdenoCA & Infiltrating duct carcinoma; Medullary; Mucinous & 198 & 197 & 1 & 56 & $39-76$ \\
\hline Breast & Breast-DCIS & Duct micropapillary carcinoma & 3 & 3 & 0 & 55 & $43-60$ \\
\hline Breast & Breast-LobularCA & Lobular carcinoma & 13 & 13 & 0 & 53 & $42-69$ \\
\hline Total & & & 2658 & 1189 & 1469 & 59 & 21-76 \\
\hline
\end{tabular}

Adeno., adenocarcinoma; Ca., carcinoma; Comb., combined; F, female; HCC, hepatocellular carcinoma; M, male; Med, median; 10-90th, 10-90th centiles; SCC, squamous cell carcinoma. 


\section{Ethical Considerations of Genomic Cloud Computing}

The PCAWG project represents the first large-scale use of distributed cloud computing in genomics. The project involved the movement of large quantities of personal health information across multiple legal jurisdictions and responsible use of this data by several hundred international researchers. Donor consents were written to explicitly allow for broad research use of the data and for international data sharing. PCAWG was granted permission by the leads of each of the tumour data providers to store, analyse and distribute the data on academic and/or commercial compute clouds.

To ensure that the PCAWG personal data were handled in a manner consistent with the donor consents, authorised representatives of each of the academic clouds and high-performance computing facilities signed a commitment not to access controlled tier data beyond the minimum needed to administer it. We negotiated similar contractual terms with commercial cloud partners. Prior to accessing the data, each PCAWG researcher was required to obtain local Institutional Review Board approval for their proposed analytic projects, and obtained controlled tier authorisation from dbGaP (National Center for Biotechnology Information) and the ICGC DACO (Centre of Genomics and Policy at McGill University). To handle the data securely, we encrypted it while in motion and at rest. We used a central authentication and digital token generating system to enforce a strong data access protocol that required researchers to provide their TCGA and/or ICGC credentials prior to accessing controlled tier data. No data breach or other compromise of donor confidentiality is known to have occurred over the course of the PCAWG project, despite its extensive use of cloud computing. 


\section{Extended Data Table 3 | Scientific output using PCAWG data, in bite-size chunks}

\begin{tabular}{ll}
\hline Scientific area & Key findings \\
\hline Driver mutations & \\
\hline $\begin{array}{l}\text { Discovery of non- } \\
\text { coding drivers }\end{array}$ & $\begin{array}{l}\text { C Estimated 10-fold more coding than non-coding driver point mutations. } \\
\text { processes than selection. }\end{array}$ \\
\hline $\begin{array}{ll}\text { Drivers by pathways } \\
\text { and networks }\end{array}$ & $\begin{array}{l}\text { - Both coding and non-coding alterations contribute to cancer pathways. } \\
\text { - Some pathways, such as RNA splicing, are primarily driven by non-coding mutations. }\end{array}$ \\
\hline Evolution and heterogeneity & 16
\end{tabular}

- Each tumour type has a distinct pattern of early and late-occurring driver events.

Timing of cancer

- Earliest somatic mutations may occur decades prior to diagnosis, providing opportunities evolution for early diagnosis.

- Intra-tumour heterogeneity is widespread and tumour subclones contain drivers that are under positive selection.

\section{Structural variants}

Patterns of structural variation

- Replication-based mechanisms of genome rearrangement frequent in many cancers, often causing driver structural variants.

- 16 signatures of SV, including break-and-ligate patterns and copy-and-insert patterns, varying by size range, replication timing, tumour type and patient.

Functional consequence of structural variation

Patterns of retrotransposition

- 52 regions with recurrent structural breakpoints and 90 recurrently fused pairs of loci show evidence of positive selection.

- Oncogenic fusions are shaped by juxtaposition of proto-oncogenes with tissue-specific regulatory elements.

- Many flavours of somatic retrotransposition in many cancers: LINE element mobilisation; transductions, pseudogenes, Alu elements.

- Retrotranspositions can induce genomic instability, including large deletions and 10 breakage-fusion-bridge cycles amplifying cancer genes.

- Chromothripsis pervasive across cancers, with frequency $>50 \%$ in several tumour types.

- Replicative processes and templated insertions contribute to rearrangement.

\begin{tabular}{lll}
\hline Chromothripsis & $\begin{array}{l}\text { - Chromothripsis pervasive across cancers, with frequency }>50 \% \text { in several tumour types. } \\
\text { - Replicative processes and templated insertions contribute to rearrangement. }\end{array}$ & 18 \\
\hline Mutational signatures & & \\
\hline $\begin{array}{l}\text { Signatures of point } \\
\text { mutations }\end{array}$ & $\begin{array}{l}\text { - Multiple signatures from unknown processes of DNA damage, repair and replication. } \\
\text { Mutation distribution }\end{array}$ & $\begin{array}{r}\text { - Uneven distribution of somatic mutations and structural variants across the genome } \\
\text { explained by epigenetic state of tissue, cell of origin and topological associated domains. }\end{array}$ \\
across genome & - Can be used to identify a tumour's type and presumed tissue/cell of origin.
\end{tabular}

\section{Transcriptional consequences of somatic mutation}

- Genomic basis for RNA alterations across 1200 tumours, including quantitative trait loci, RNA effects of somatic allele specific expression and alternative splicing.

mutation $\quad$ Link between mutational signatures and expression; classification of gene fusions;

identification of genes recurrently altered at RNA level.

\section{Others}

\begin{tabular}{lcl}
\hline $\begin{array}{l}\text { Tumour subtypes from } \\
\text { genome sequencing }\end{array}$ & $\begin{array}{c}\text { - Genomic distribution of somatic mutations, mutational signatures and driver mutations } \\
\text { accurately distinguish major tumour types of primaries and metastases. }\end{array}$ & 12 \\
\hline $\begin{array}{l}\text { Mitochondrial DNA } \\
\text { mutations }\end{array}$ & $\begin{array}{l}\text { - Somatic mitochondrial truncating mutations frequent in certain cancer types, associated } \\
\text { with activation of critical signaling pathways. }\end{array}$ & 14 \\
\hline $\begin{array}{l}\text { Telomere biology and } \\
\text { sequences }\end{array}$ & $\begin{array}{l}\text { - Activating TERT promoter mutations are the single most frequent non-coding driver. } \\
\text { In ATRX/DAXX-mutant tumours, aberrant telomere variant repeat distribution is } \\
\text { common. }\end{array}$
\end{tabular}




\section{natureresearch}

Peter Campbell, Joshua Stuart, Gad Getz, Jan

Corresponding author(s): Korbel, Lincoln Stein

Last updated by author(s): Oct 23, 2019

\section{Reporting Summary}

Nature Research wishes to improve the reproducibility of the work that we publish. This form provides structure for consistency and transparency in reporting. For further information on Nature Research policies, see Authors \& Referees and the Editorial Policy Checklist.

\section{Statistics}

For all statistical analyses, confirm that the following items are present in the figure legend, table legend, main text, or Methods section.

n/a Confirmed

The exact sample size $(n)$ for each experimental group/condition, given as a discrete number and unit of measurement

A statement on whether measurements were taken from distinct samples or whether the same sample was measured repeatedly

The statistical test(s) used AND whether they are one- or two-sided

Only common tests should be described solely by name; describe more complex techniques in the Methods section.

$\bigotimes$ A description of all covariates tested

A description of any assumptions or corrections, such as tests of normality and adjustment for multiple comparisons

A full description of the statistical parameters including central tendency (e.g. means) or other basic estimates (e.g. regression coefficient)

AND variation (e.g. standard deviation) or associated estimates of uncertainty (e.g. confidence intervals)

For null hypothesis testing, the test statistic (e.g. $F, t, r$ ) with confidence intervals, effect sizes, degrees of freedom and $P$ value noted

Give $P$ values as exact values whenever suitable.

For Bayesian analysis, information on the choice of priors and Markov chain Monte Carlo settings

For hierarchical and complex designs, identification of the appropriate level for tests and full reporting of outcomes

Estimates of effect sizes (e.g. Cohen's $d$, Pearson's $r$ ), indicating how they were calculated

Our web collection on statistics for biologists contains articles on many of the points above.

\section{Software and code}

\section{Policy information about availability of computer code}

Data collection

Data and metadata were collected from International Cancer Genome Consortium (ICGC) consortium members using custom software packages designed by the ICGC Data Coordinating Centre. The general-purpose core libraries and utilities underlying this software have been released under the GPLv3 open source license as the "Overture" package and are available at https://www.overture.bio. Other data collection software used in this effort, such as ICGC-specific portal user interfaces, are available upon request to contact@overture.bio.

Data analysis The workflows executing core WGS alignment, QC and variant-calling software are packaged as executable Dockstore images and available at: https://dockstore.org/search?labels.value.keyword=pcawg\&searchMode=files. Individual software components are as follows: BWA-MEM v0.78.8-r455; DELLY v0.6.6; ACEseq v1.0.189; DKFZ somatic SNV workflow v1.0.132-1; Platypus v0.7.4; ascatNgs v1.5.2; BRASS v4.012; grass v1.1.6; CaVEMan v1.50; Pindel v1.5.7; ABSOLUTE/JaBbA v1.5; SvABA 2015-05-20; dRanger 2016-03-13; BreakPointer 2015-12-22; MuTect v1.1.4; MuSE v1.Orc; SMuFIN 2014-10-26; OxoG 2016-4-28; VAGrENT v2.1.2; ANNOVAR V2014Nov12; VariantBAM v2017Dec12; SNV-Merge v2017May26; SV-MERGE V2017Dec12; DKFZ v2016Dec15; Chromothripsis Explorer v1.0.

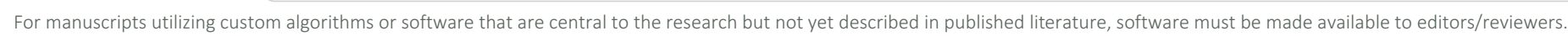
We strongly encourage code deposition in a community repository (e.g. GitHub). See the Nature Research guidelines for submitting code \& software for further information.

\section{Data}

Policy information about availability of data

All manuscripts must include a data availability statement. This statement should provide the following information, where applicable:

- Accession codes, unique identifiers, or web links for publicly available datasets

- A list of figures that have associated raw data

- A description of any restrictions on data availability

WGS somatic and germline variant calls, mutational signatures, subclonal reconstructions, transcript abundance, splice calls and other core data generated by the ICGC/TCGA Pan-cancer Analysis of Whole Genomes Consortium are available for download at https://dcc.icgc.org/releases/PCAWG. Additional information on accessing the data, including raw read files, can be found at https://docs.icgc.org/pcawg/data/. In accordance with the data access policies of the ICGC and TCGA 
projects, most molecular, clinical and specimen data are in an open tier which does not require access approval. To access potentially identification information, such as germline alleles and underlying sequencing data, researchers will need to apply to the TCGA Data Access Committee (DAC) via dbGaP (https:// dbgap.ncbi.nIm.nih.gov/aa/wga.cgi?page=login) for access to the TCGA portion of the dataset, and to the ICGC Data Access Compliance Office (DACO; http:// icgc.org/daco) for the ICGC portion. In addition, to access somatic single nucleotide variants derived from TCGA donors, researchers will also need to obtain dbGaP authorization

\section{Field-specific reporting}

Please select the one below that is the best fit for your research. If you are not sure, read the appropriate sections before making your selection.

\section{Life sciences study design}

All studies must disclose on these points even when the disclosure is negative.

Sample size

We compiled an inventory of matched tumour/normal whole cancer genomes in the ICGC Data Coordinating Centre. Most samples came from treatment-naïve, primary cancers, but there were a small number of donors with multiple samples of primary, metastatic and/or recurrent tumours. Our inclusion criteria were: (i) matched tumour and normal specimen pair; (ii) a minimal set of clinical fields; and (iii) characterisation of tumour and normal whole genomes using Illumina HiSeq paired-end sequencing reads.

We collected genome data from 2,834 donors, representing all ICGC and TCGA donors that met these criteria at the time of the final data freeze in autumn 2014. No statistical methods were used to predetermine sample size.

Data exclusions After quality assurance, data from 176 donors were excluded as unusable. Reasons for data exclusions included inadequate coverage, extreme bias in coverage across the genome, evidence for contamination in samples and excessive sequencing errors (for example, through 8oxoguanine). Exclusion criteria were pre-determined.

Replication In order to evaluate the performance of each of the mutation-calling pipelines and determine an integration strategy, we performed a largescale deep sequencing validation experiment. We selected a pilot set of 63 representative tumour/normal pairs, on which we ran the three core pipelines, together with a set of 10 additional somatic variant-calling pipelines contributed by members of the SNV Calling Working Group. Overall, the sensitivity and precision of the consensus somatic variant calls were 95\% (Cl90\%: 88-98\%) and 95\% (Cl90\%: 71-99\%) respectively for SNVs. For somatic indels, sensitivity and precision were 60\% (34-72\%) and 91\% (73-96\%) respectively. Regarding SVs, we estimate the sensitivity of the merging algorithm to be $90 \%$ for true calls generated by any one caller; precision was estimated as $97.5 \%$ - that is, $97.5 \%$ of SVs in the merged SV call-set have an associated copy number change or balanced partner rearrangement.

Randomization No randomisation was performed - this was a descriptive study, not an experimental study.

Blinding

No blinding was undertaken - this was a descriptive study, not an experimental study.

\section{Reporting for specific materials, systems and methods}

We require information from authors about some types of materials, experimental systems and methods used in many studies. Here, indicate whether each material, system or method listed is relevant to your study. If you are not sure if a list item applies to your research, read the appropriate section before selecting a response.

Materials \& experimental systems

$\mathrm{n} / \mathrm{a}$ Involved in the study

Methods

\ $\square$ Antibodies

Х $\square$ Eukaryotic cell lines $\mathrm{n} / \mathrm{a}$ Involved in the study Х $\square$ Palaeontology

Х ChIP-seq

Х $\square$ Animals and other organisms

Х $\square$ Flow cytometry

X $\square$ MRI-based neuroimaging

$\square \bigotimes$ Human research participants

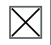
Clinical data

\section{Human research participants}

Policy information about studies involving human research participants

Population characteristics

Patient-by-patient clinical data are provided in the marker paper for the PCAWG consortium (Extended Data Table 1 of that manuscript). Demographically, the cohort included 1,469 males (55\%) and 1,189 females (45\%), with a mean age of 56 years (range, 1-90 years). Using population ancestry-differentiated single nucleotide polymorphisms (SNPs), the ancestry distribution was heavily weighted towards donors of European descent (77\% of total) followed by East Asians (16\%), as expected for large contributions from European, North American and Australian projects. We consolidated histopathology descriptions of the tumour samples, using the ICD-0-3 tumour site controlled vocabulary. Overall, the PCAWG data set comprises 38 distinct tumour 
types. While the most common tumour types are included in the dataset, their distribution does not match the relative population incidences, largely due to differences among contributing ICGC/TCGA groups in numbers sequenced.

Recruitment

Patients were recruited by the participating centres following local protocols. Samples obtained had to meet criteria on amount of tumour DNA available, meaning that the cohort is potentially somewhat biased towards larger tumours. Otherwise, we anticipate no major recruitment biases.

Ethics oversight

The Ethics oversight for the PCAWG protocol was undertaken by the TCGA Program Office and the Ethics and Governance Committee of the ICGC. Each individual ICGC and TCGA project that contributed data to PCAWG had their own local arrangements for ethics oversight and regulatory alignment.

Note that full information on the approval of the study protocol must also be provided in the manuscript. 